\title{
EFFECTS OF THE MAXIMUM CREDIBLE ACCIDENT. RELEVANT TO THE DESIGN \\ OOF THE \\ CONTAINMENT SHELL \\ EXPERIMENTAL LOW-TEMPERATURE PROCESS HEAT REACTOR PROJECT
}

\section{4}

MASTER

March 21, 1960

Prepared under Contract AT $(11-1)-832$

by the

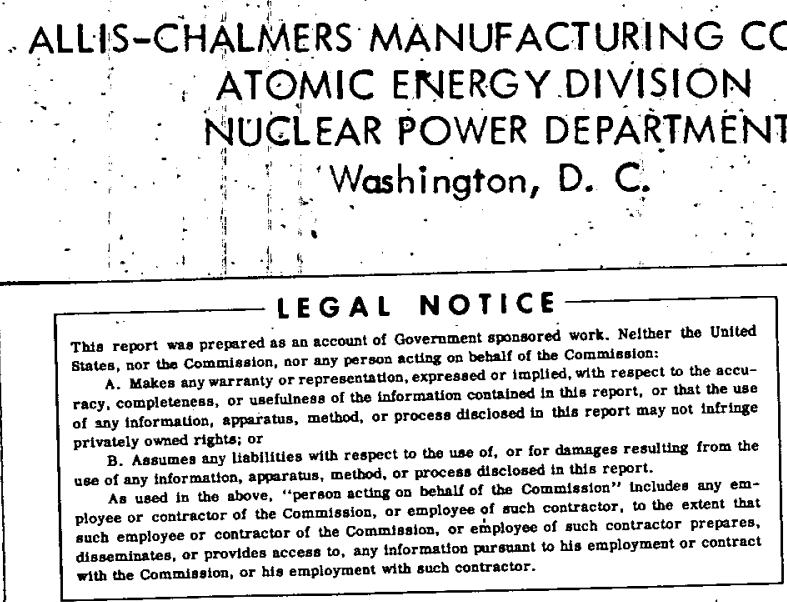




\section{DISCLAIMER}

This report was prepared as an account of work sponsored by an agency of the United States Government. Neither the United States Government nor any agency Thereof, nor any of their employees, makes any warranty, express or implied, or assumes any legal liability or responsibility for the accuracy, completeness, or usefulness of any information, apparatus, product, or process disclosed, or represents that its use would not infringe privately owned rights. Reference herein to any specific commercial product, process, or service by trade name, trademark, manufacturer, or otherwise does not necessarily constitute or imply its endorsement, recommendation, or favoring by the United States Government or any agency thereof. The views and opinions of authors expressed herein do not necessarily state or reflect those of the United States Government or any agency thereof. 


\section{DISCLAIMER}

Portions of this document may be illegible in electronic image products. Images are produced from the best available original document. 
1. INTRODUCTION.

2. CONCLUSIONS.

2.1 Definition of the Maximum Credible Accident

2.2 Thermal and Hydraulic Effects (Other Than Meltdown) of the Maxinum Credible Accident.

2.3 Extent of Cladding Meltdown

2.4 Energy Released From The Reactor System

2.5 Extent of Fission-Product Release To The Contalnment shell

3. ASSUMPTIONS.

3.1 Thermal and Hydraulic Effects (Other Than Meltdown) of the Maximum Credible Accident

3.2 Extent of cladding Meltdown

3.3 Energy Released From The Reactor System

3.4 Extent of Fission-Product Release To The Contalnment Shell

4. DISCUSSION...

APPENDIX I - ANALYSIS OF THERMAL AND HYDRAULIC EFFECTS (OTHER THAN MELTDOWN) OF THE ELPHR MAXIMUM CREDIBLE ACCIDENT ON THE REACTOR SYSTEM

APPENDIX II - ANALYSIS OF THE EXTENT OF CLADDING MELTDOWN AFTER THE ELPHR MAXIMUM CREDIBLE ACCIDENT

APPENDIX III - ANALYSIS OF THE ENERGY RELEASED FROM THE REACTOR SYSTEM AFTER THE ELPHR MAXIMUM CREDIBLE ACCIDENT

APPENDIX IV - ANALYSTS OF THE EXTENT OF FISSION-GAS - RELEASE AFTER THE ELPHR MAXIMUM CREDIBLE ACCIDENT 


\section{INTRODUCTION}

To demonstrate that the contalnment shell of a nuclear reactor power plant is properly designed, it must be shown

- (1) that the Internal pressure it can withstand does not exceed the pressure rise assoclated w1th the maximum credible accident,

(2) that the fission products released by the maximum credible accident do not represent an unacceptably intense source of direct radiation at points outside the sheli, and

(3) that the gaseous and volatile fission products released by the maximum credible accident do not escape from the shell fast enough to cause unacceptable risks of exposure to radiation at points outside the shell even under adverse meteorological conditions.

This analysis deals with all three points just enumerated, but it is complete only with regard to the first, $1 . e .$, the adequacy of the internal pressure that the shell is designed to withstand. Regarding the second and third points, this analysis includes a thorough and detalled estimate of the extent to which fission products would escape from the reactor core into the contalnment shell, but does not go further. With the data presented herein, however, the usual methods for calculating the intensity of direct radiation from the shell and of the effects of fission-product leakage from the shell may be applied. It 1s expected that Sargent \& Lundy w1ll complete the work necessary to demonstrate that the radiation hazards resulting from the maximum credible accldent are acceptably low.

This report, therefore, is a complete basis for judging the contalnment shell pressure rating, but only a partial basis for judging the necessity of shadow shielding and the acceptability of the allowable leakage rate.

The preliminary design of the containment shell is described in Sec. VI of the ELPHR Site Evaluation Report (SL-1760), pp. 20-23. The shell encloses a free-air volume of approximately $100,000 \mathrm{cu}$. ft., and $1 \mathrm{~s}$ designed for a working pressure of 31 psig. It will be tested at a maximum pressure of $39 \mathrm{ps} 1 \mathrm{~g}$. The maximum allowable gaseous leakage from it in any $24-\mathrm{hr}$. period after pressurization to 31 psig is 0.10 per cent of its free-air volume. 
Assessing the sultability of the containment shell involves seven speclflc tasks:

(1) defining the maximum credible accident;

(2) analyzing the thermal and hydraulic effects (other than meltdown) of the maximum credible accident on the reactor system;

(3) estimating the extent to which the fuel-rod cladding will melt as a result of the maximum credible accident;

(4) estimating the amount of energy released from the reactor system by the escaping steam and water and by a possible chemical reaction, and the corresponding pressure rise inside the containment shell;

(5) estimating the kinds, amounts, and total radioactivity of fission products released to the atmosphere of the contalnment sheli after the core melts;

(6) estimating the direct gamma-ray dose from the shell after radioactivity is released to its interior; and

(7) estimating the results of the escape of gaseous radionuclides and alrborne radioactive particles from the containment shell at a rate determined by the maximum allowable leakage rate.

As implied by the earlier statements, this report presents conclusions regarding only the first five tasks; the remaining two w11l be completed by Sargent \& Lundy. Appendixes I, II, III, and IV contain the detalled calculations carried ou't for Tasks $2,3,4$, and 5 .

In addition to the spec1fic assumptions set forth in Sec. 3 of this report, it was assumed throughout that

(1) the reactor core consists of 32 fuel assemblies,

(2) each fuel assembly consists of a $10 \times 10$ square array of 100 rods of which all except the corner rods contain fuel and are clad with pure aluminum (the corner rods are assumed to be empty stalnilesssteel tubes),

(3) the reactor power level is $40.1 \mathrm{MW}$, and

(4) the full-power core lifetime is 1.45 years. 
2. CONCLUSIONS

2.I Definition of the Maximum Credible Accident

The most serlous ELPHR accldent whose occurrence is considered possible is an instantaneous gross rupture of the 18-in. coolant inlet pipe just outside the b1ologlcal shield. The accldent allows the unimpeded escape of water and steam from the broken pipe on both sides of the mupture, 1.e., directly from the reactor vessel inlet pipe on the biological-shield side of the mupture and from the rest of the primary system on the other side.

2.2 Thermal and Hydraul1c Effects (Other Than Meltdown) of the Maximum Credible Accident

Twelve seconds after the maximum credible accident all water w111 have been expelled from the reactor vessel. The average fuel-cladding temperature, the average coolant temperature inside the reactor vessel, and the fraction of the reactor-vessel volume occupied by water are plotted as functions of time during this 12sec. interval in FIg. 1 .

The average coolant temperature inside the steam generator and the fraction of the primary-side volume inside the steam generator occupled by water are plotted as functions of time after the accident in Fig. 2 .

The average temperature inside the pressurlzer and the fraction of the pressurizer volume occupied by water are plotted as functions of time after the accident in FIg. 3 .

The mass flow rates from each side of the rupture are plotted as functions of time after the accident in F1g. 4.

These conclusions are supported by the calculations presented in Appendix I.

2.3 Extent of Cladding Meltdown

Four minutes after all water is expelled from the reactor vessel, or 4.2 min. after the maximum credible accldent, the cladding of the hottest fuel rods will reach the melting point (1219 F). Subsequently the cladding on all fuel rods except those on the periphery 
of each fuel assembly will melt. The cladding of the fuel rods on the periphery of each fuel assembly will not reach the meliting point at all.

There is a $10 \times 10$ array of rods in each fuel assembly. All contain fuel except the comier ones. Accordingly, each fuel assembly has 32 fuel rods on the perlphery. Therefore; the ratio of the number of fuel rods whose cladding w1I melt to the total number of fuel rods is $(96-32) / 96$, of $2 / 3$. These conclusions are supported by the daIculations THIS WAS
presented in Appendix II.

2.4 Energy Released From The Reactor System

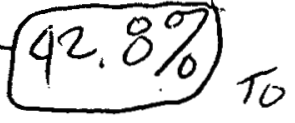
CHANGED A $5 \times 5$

The energy released from the reactor system as a result of the maximum credible accident was estimated for primary-system pressures of 900 psia and 300 psia. Even though the aluminum-clad core may not be operated at 900 psia, the higher pressure was assumed to Insure that the most severe conditions possible were taken into account. In each case it was assumed that 10 per cent of the molten aluminum cladding ( 6.67 per cent of all aluminum in the core) reacts with water.

The energy released by the maximum credible accident followed by the aluminum-water reaction will raise the pressure in the containment sheli to 31.9 psig if the primary-system pressure was initially 900 psia, and to $16.7 \mathrm{psig}$ if the primary-system pressure was initially $300 \mathrm{psia}$. As mentioned in the introduction, the working pressure for which the contalnment shell is designed $1 s 31$ psig.

These conclusions are supported by the calculations presented in Appendix III.

2.5 Extent of Fission-Product Release To The Containment Shell

The radionuclides released by the melting: of the cladding of two-thirds of all fuel rods have a gross activity of $365^{\prime} \mathrm{kllocuries.} \mathrm{This} \mathrm{is} \mathrm{two-thirds} \mathrm{of} \mathrm{all}$ activity that would be released ( $552 \mathrm{kilocurles}$ ) if the cladding on all fuel rods melted. Table 1 lists the activity of each of the principal radionuclides that would be released if all cladding melted. 


\section{TABLE 1}

ACTIVITY OF PRINCIPAL RADIONUCLIDES THAT WOULD BE RELEASED FROM THE ELPHR CORE IF ALL FUEL-ROD CLADDING MELTED

Radionuclide

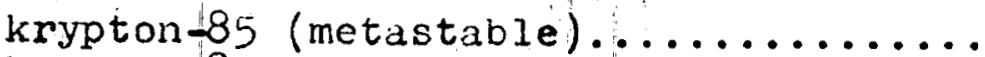

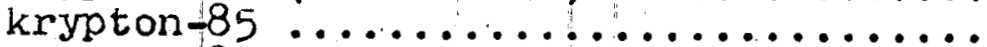

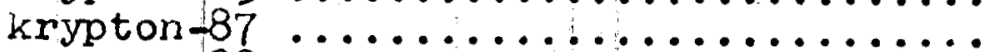

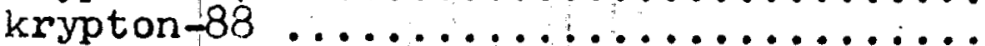

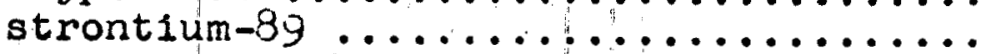
stront1um $-90 \ldots \ldots \ldots \ldots \ldots \ldots$

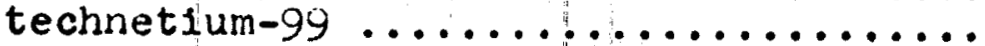

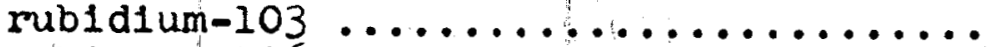

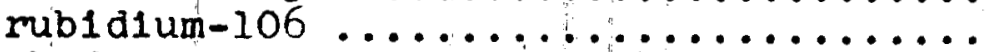
rhodium-105 antimony -125 ant1mony-127 Activity (kilocuries)

tellurium-129

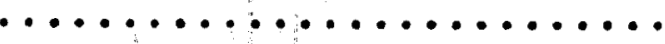

tellurium-132
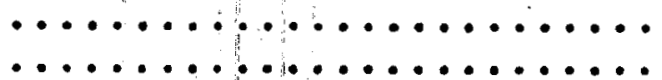
lodine-131 lodine -133 $\ldots \ldots \ldots \ldots \ldots \ldots \ldots \ldots \ldots \ldots \ldots$

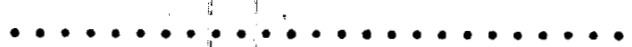
iodine -135

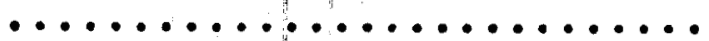
xenon-131 xenon -133 xenon-135
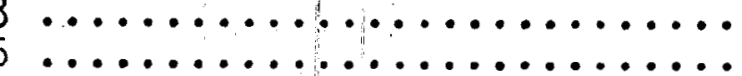
cesium-137 cesium -138 barium-140
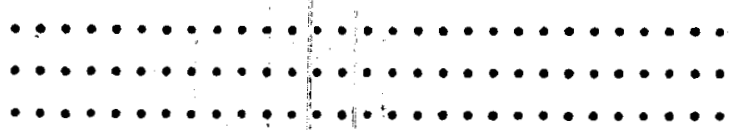

TOTAL
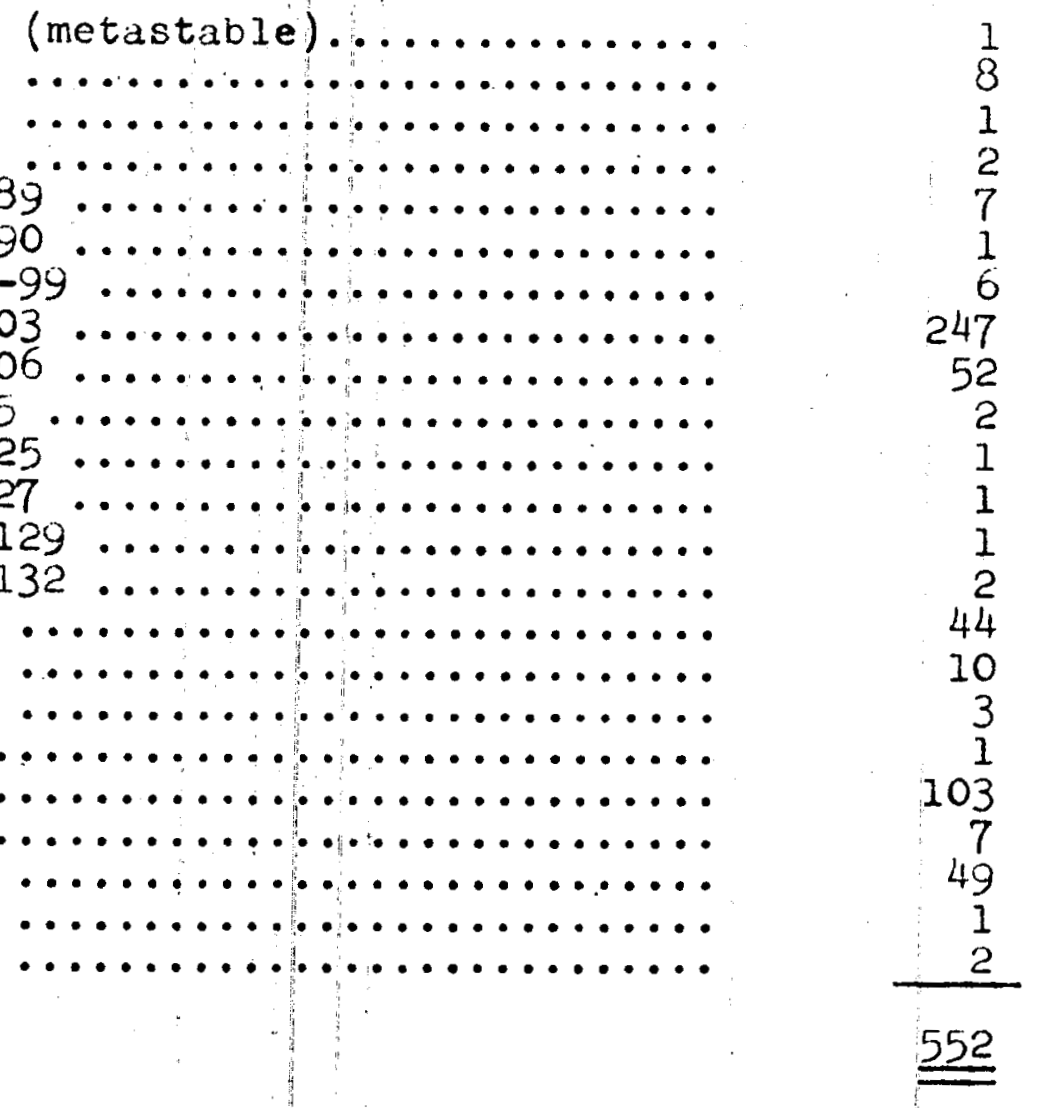

These conclusions are supported by the calculations presented in Appendix IV.

Section VIII, pp 25-33 of The ELPHR Site Evaluation Report (SL-167O) presents estimates of effects of releasing 25 per cent of all fission products formed after 400 days of operation at full power. All source strengths calculated on this basis are considerably higher than those listed above; therefore, the direct-radiation, whole-body, inhalation, and grounddeposition doses to be caiculated on the basis of Table 1 will be significantly lower than those appearing in the site Evaluation Report.

\section{ASSUMPTIONS}

3.1 Thermal and Hydraulic Effects (Other Than Meltdown) of the Maximum Credibie Accident

Analysis of the thermal and hydraulic effects (other than meltdown) of the maximum credible accident proceeded on the basis of nine assumptions: 
(1) The rupture occurs instantaneously in the cold leg, immediately outside the blological shield.

(2) The inlet duct inside the reactor vessel collapses without plugging the inlet nozzle.

(3) The coolant escapes from the pipe ends on each side of the rupture at sonic velocity.

(4) The reactor coolant system is 1dealized into several volumes and fluld-flow resistances as follows: the reactor vessel, the pressurizer, and the s.team generator are considered as volumes; both sides of the ruptured leg, the pipe between pressurizer and the hot leg, and the hot leg leading from the reactor vessel to the steam generator are considered as fluidflow resistances (see sketch, Appendix I).

(5) Water is considered as an incompressible fluid and its vapor as an ideal gas.

(6) Saturation conditions prevali in the reactor coolant system after the accident.

(7) OnIy adiabatic processes are considered, except in the reactor vessel and steam generator.

(8) The temperature of the secondary coolant legving the steam generator remains constant at $250^{\circ} \mathrm{F}$.

(9) Pressure variations due to afferent elevations are disregarded.

3.2 Extent of Cladding Meltdown

Analysis of the extent to which the fuel-rod cladding would melt as a consequence of the maximum credible accident proceeded on the basis of six assumptions:

(1) The initlal conditions for the meltdown analysis are the conditions existing $12 \mathrm{sec}$. after gross failure of the 18-in. inlet line, 1.e., when there is no longer any water in the reactor vessel.

(2) Water at $70^{\circ} \mathrm{F}$. starts to be injected into the reactor vessel lat a rate of $40 \mathrm{gpm} 12 \mathrm{sec}$. after the pipe fallure; the water leaves the reactor vessel through the miptured inlet line at the boiling point ( $212^{\circ}$ F.). 
(3) The radial distribution of the 40 gpm flow of cooling water leaving the spray nozzles is the same as the radial distribution of heat generation in the reactor core.

(4) The water injected into the core removes heat only from the peripheral fuel rods in each fuel assembly.

(5) The only process by which heat is removed from all other rods in each fuel assembly is radiation.

(6) No metal-water reaction takes place; $1 . e .$, decay

of radionuclides is the only source of heat considered.

3.3 Energy Released From The Reactor System

The energy released from the reactor system was estimated on the basis of three assumptions:

(1) Two-thirds of the aluminum in the core melts; of this, approximately 10 per cent reacts exothermically with water.

(2) The amblent temperature inside the containment shell $18,100^{\circ} \mathrm{F}$.

(3) The water in the primary system is instantaneousiy released and flashes to steam adiabatically; 1.e., heat is absorbed by the air inside the containment shell and none is transferred outside or stored by transfer to the stmuctures inside.

The first assumption is conservative because it is unlikely that as much as 10 per cent of the molten aluminum will react; in fact, it is doubtful whether any aluminum will react. This statement is based on experiments by the Aerojet General. Corporation reported in "A Study of the Reaction of Metal and Water" (AECD3664) and "The Reaction of Molten Uranium and ZirconIum Alloys with Water" (AOC-AE-17).

3.4 Extent of Fission-Product Release to the Containment Shell

The extent of fission-product release to the containment shell was estimated by applying the method set forth in the Experimental Gas-Cooled Reactor Freliminary Hazards Summary Report (ORO-196), Sec. 7.2.1, pp. $7.2-7.6$. 
4. DISCUSSION

$$
-8-
$$

Because the design of the reactor and the analysis work reported herein proceeded concurrently, more accurate data concerning the reactor became available from time to time. The calculations reflect the later data wherever feasible, but some inconsistencies deserve comment.

Appendixes I and II were prepared first, on the basis of the most reliable estimate: of primary-system volume (603 cu.ft.) then available. operation of the aluminumclad core at $900 \mathrm{psia}$ and $415^{\circ} \mathrm{F}$. was not envisioned; accordingly, the analysis presupposed operation at 300 psla and $285^{\circ} \mathrm{F}$. only. At the lower pressure and temperature and assuming a primary-system volume of 603 cu.ft., the total mass of steam and water in the primary system is approximately 34,800 pounds.

When Appendix III was prepared, a more nearly accurate estimate of the primary system volume was avallable ( 686 cu.ft.), and operation of the aluminum-clad core at 900 psia and $415^{\circ} \mathrm{F}$. was at least a possibility. With a volume of $686 \mathrm{cu}$.ft!., the primary system contains approximately 38,300 pounds of steam and water at 300 psia and 285 F., and approximately 35,200 pounds of steam and water at 900 psia and $415^{\circ} \mathrm{F}$.

The calculations presented in Appendixes I and II were not revised to account for the larger volume and higher pressure and.temperature because it can be shown that although these changes in initial conditions affect the length of the interval between the accident and exposure of the core, they do not affect the extent to which the cladding melts. This is true because the temperature of the peripheral fuel rods continuously decreases after the accldent; only that of the interior fuel rods rises. Variations in the length of time before the core is exposed represented by the changes discussed here are not significant.

However, the larger volume and higher pressare and temperature strongly affect the pressure rise in the containment she11; therefore, Appendix III was prepared on the basis of a primary-system volume of 686 cu. ft. and both sets of operating conditions. 


\section{APPENDIX I}

ANALYSIS OF THERMAL AND HYDRAULIC EFFECTS (OTHER THAN MELTDOWN) OF THE ELPHR MAXIMUM CREDIBLE ACCIDENT ON THE REACTOR SYSTEM

\section{CONTENTS}

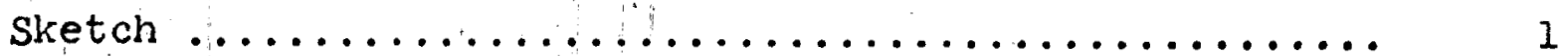

Der1vation of Equations $\ldots \ldots \ldots \ldots \ldots \ldots \ldots \ldots \ldots \ldots \ldots \ldots \ldots$

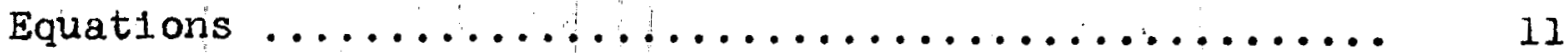

Constants ............................ 18

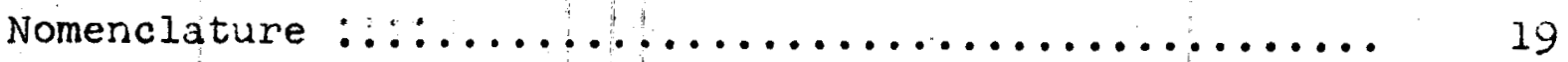

Addendum: Reduction in the number of variables

Elimination of Vamiables ................... I

Final set of Equations .................. 6 


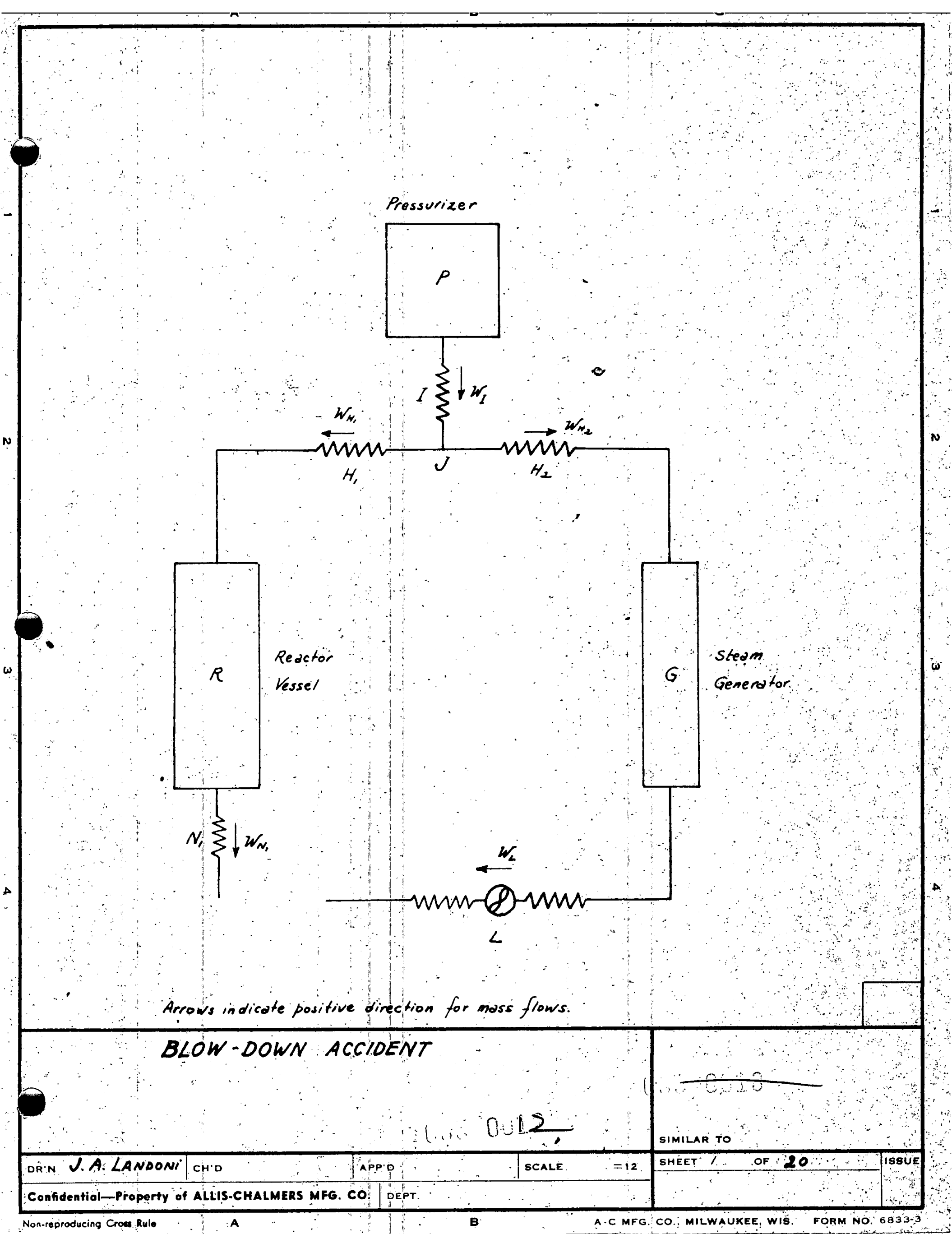




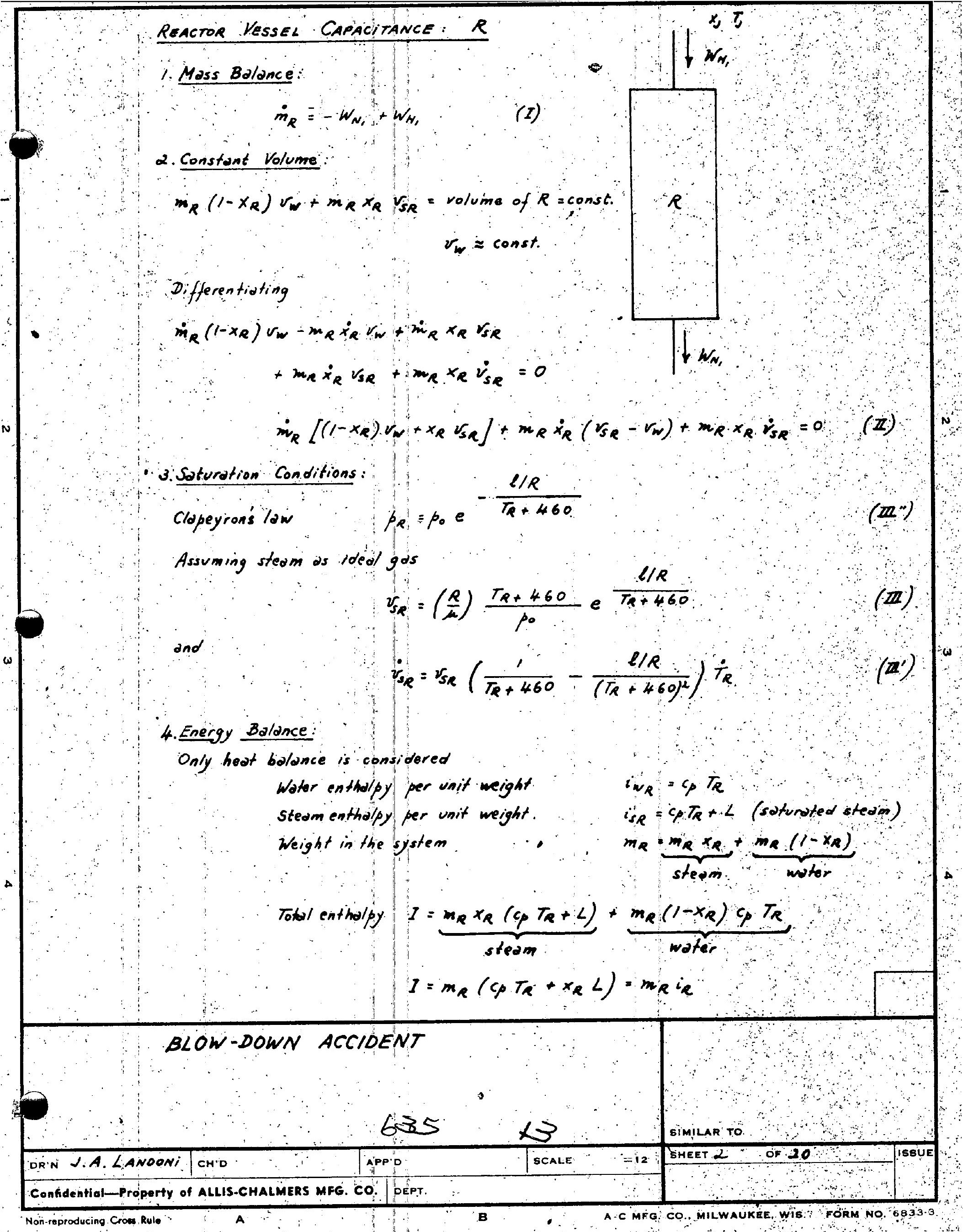


Differentiating

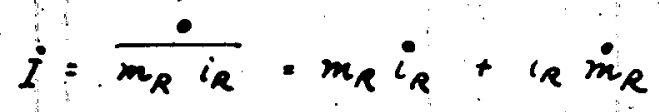

$\frac{\dot{m}}{m_{R} T_{R}}=\dot{m}_{R}\left(c_{p} T_{R}+x_{R} L\right)+m_{R}\left(c_{p} \dot{T}_{R}+\dot{x}_{R} L\right)$

$m_{R} i_{R}=m_{R}\left(\frac{Q}{m_{R}}+\frac{1}{J} r_{R} \dot{p}_{R}\right)=Q+m_{R} v_{R} \dot{p}_{R} \frac{1}{J}$

(If adiabatic process $Q=0$ and $m i_{R}=\frac{h_{m}}{J} v_{R} p_{R}$ )

Assuming

$$
Q=V_{R}\left(1-x_{R}\right)\left(\bar{T}_{0}-T_{R}\right) \frac{V_{m}}{V_{R}}
$$

Thus

$$
m_{R} i_{R}=\dot{J}_{R} v_{R} \dot{p}_{R}+v_{R}\left(1-x_{R}\right)\left(T_{0}-T_{R}\right)
$$

If $W_{M}>0$

$$
\sum i_{R} \dot{m}_{R}=-w_{N_{1}}\left(c_{p} T_{R}+x_{R} l\right)+w_{N_{1}}\left(c_{p} T_{g}+x_{J} l\right)
$$

If $w_{N_{1}}<0$

$\sum i_{R} \dot{m}_{R}=-\left(w_{N_{1}}-w_{H_{1}}\right)\left(C_{P} T_{R}+x_{R} L\right)$

Equoting:

If $w_{N_{1}} \geq 0 ; \quad \dot{m_{R}}\left(c_{p} T_{R}+x_{R} L\right)+m_{R}\left(c_{p} \dot{T}_{R}+\dot{x}_{R} L\right) \alpha_{j} v_{R} m_{R} \beta_{R}+v_{R} v_{R}\left(1-x_{R}\right)\left(\bar{T}_{0}-T_{R}\right)$

(ID)

$$
-w_{N_{1}}\left(i_{p}, T_{R}+x_{R} L\right)+w_{N_{1}}\left(\varphi T_{j}+x_{j} L\right)
$$

If $w_{R_{1}}<0, \dot{m}_{R}\left(\varphi_{p} T_{R}+x_{R} L\right)+m_{R}\left(\varphi \dot{T}_{R}+\ddot{x}_{R} L\right)=\frac{j}{J} v_{R} m_{R} \ddot{p}_{R}+V_{R}\left(1-x_{R}\right)\left(T-T_{R}\right) \frac{v_{R}}{v_{R}}$

$$
-\left(w_{N_{1}}-w_{H_{1}}\right)\left(\phi T_{R}+x_{R} L\right)
$$

being: $W_{H},>0$ when $p_{J}>p_{e}$

$$
w_{n},<0 \quad \beta_{J}<\beta_{R}
$$

5. Specific Volume:

$$
v_{R}=\left(1-x_{R}\right) r_{w}+x_{R} v_{s e}
$$

The set of equations (I) to (V) can be worked out in order to have all the parameters explicitly written

At the beginning of each time interval the initial value of the parameters can be determined.

BLOW-DOWN ACCIDENT

1000014

\begin{tabular}{|l|l|l}
\hline & \\
\hline DR'N J.A. LANOONI & CH'D & APPO \\
\hline COnfidential-Property of ALLIS.CHALMERS MFG CO & OEPT. \\
\hline
\end{tabular}


Initial value of the parameters:

Given values:

$$
\begin{aligned}
& m_{R}(t=0)=m_{R 0} \\
& T_{R}(t=0)=T_{R_{0}} \\
& x_{R}(t=0)=x_{p_{0}} \\
& \beta_{R_{0}}=\beta_{0} e^{-\frac{l / R}{T_{R}+460}} \\
& \text { by mg (m) } \\
& v_{S R_{0}}=\left(\frac{R}{\mu_{0}}\right) \cdot \frac{T_{R}+460}{\dot{\phi}_{0}} e \frac{l / R}{T_{R}+460} \\
& \text { by eq. (III) } \\
& v_{R_{0}}=\left(1-x_{R_{0}}\right) v_{w}+x_{R_{0}} v_{R_{0}}
\end{aligned}
$$

Derivative of the parameters:

From eq. (I)

$$
\dot{m}_{R}=-W_{N_{1}}+w_{H_{1}}
$$

Differentiating eq. ( $v$ )

$$
\begin{aligned}
& \dot{v}_{R}=-\dot{x}_{R} v_{w}+\dot{x}_{R} v_{S R}+x_{R} \dot{v}_{S R} \\
& \dot{r}_{R}=\dot{x}_{R}\left(\dot{v}_{S R}-v_{w}\right)+\dot{v}_{S R} x_{R}
\end{aligned}
$$

Replacing in eq. (II) $\quad \dot{m}_{R} v_{R}+m_{R} \dot{v}_{R}=0$ (os expected from the condition of constant volume).

Thus

$$
\ddot{v}_{R}=-\frac{v_{R}}{m_{R}} \dot{m}_{R}
$$

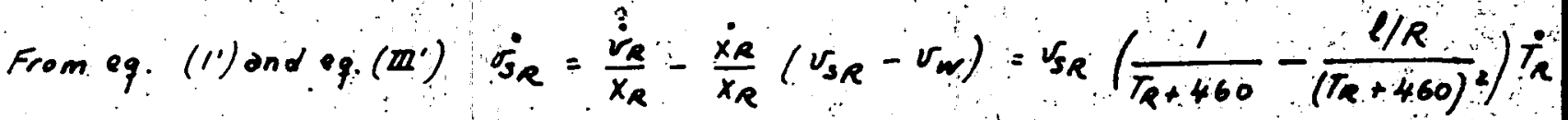

$$
\frac{v_{S R}}{T_{R}+460}\left(1-\frac{l / R}{T_{R}+460}\right) \dot{T}_{R}=\frac{\dot{V}_{R}}{x_{R}}-\frac{x_{R}}{x_{R}}\left(r_{s R}-r_{W}\right)
$$

Differentiating eq $\left(m^{m}\right) p_{R}=p_{0} e^{-\frac{\ell / R}{T_{R}+460}} \frac{l / R}{\left(T_{R}+460\right)^{2}} \dot{T}_{R}=\frac{l / R}{\left(T_{R}+460\right)^{2}} T_{R}$

$$
p_{R}=\frac{l / R}{\left.p_{R}+460\right)^{2}} \dot{T_{R}}
$$

Replacing in eq. (rV)

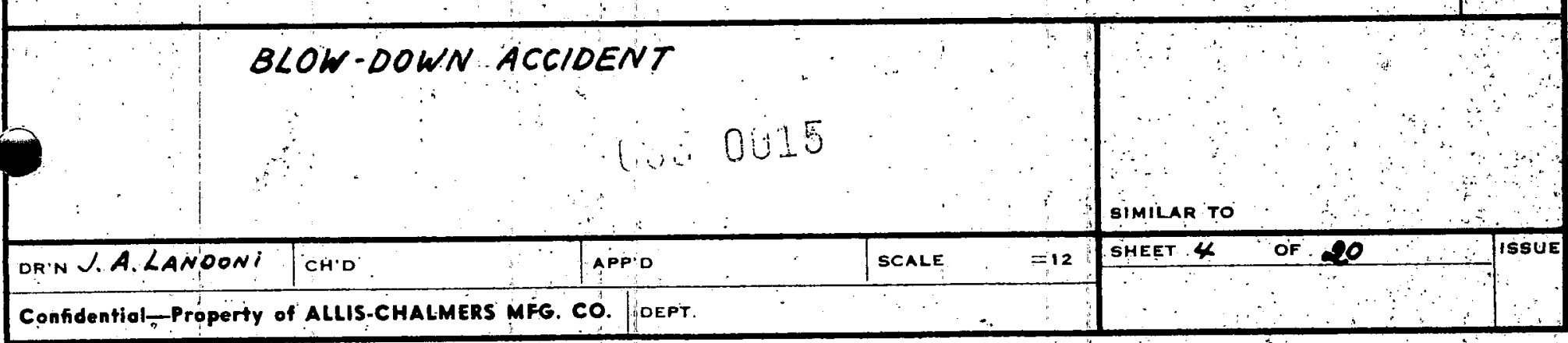

Non-reproducing Croas'Rule

$\mathbf{A}$

$\mathbf{B}$

A.C MFG. CO.: MILWAUKEE, WIS.

FORM NO. $6833-3$ 


$$
\begin{aligned}
& \dot{m}_{R}\left(c_{P} T_{R}+x_{R} L\right)+m_{R} x_{R} L+m_{R}\left(c_{P}-\frac{1}{J} v_{R} p_{R} \frac{l / R}{\left(T_{R}+460\right)^{2}}\right) \dot{T}_{R}-V_{R}\left(1-x_{R}\right)\left(T_{0}-T_{R}\right) r_{R}=
\end{aligned}
$$

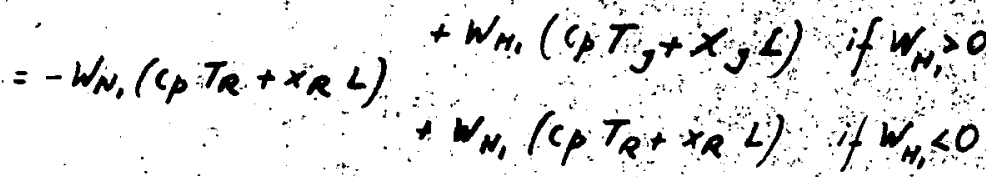

Eq (2') and eq. (3') allow us to determine $\dot{x}_{R}$ and $\dot{r}_{R}$

From eq. (2)

$$
\dot{x}_{R}=\frac{\dot{v}_{R}}{v_{S R}-v_{W}}-\frac{x_{R} v_{S R}}{T_{R}+460}\left(1-\frac{e / R}{T_{R}+460}\right) \frac{T_{R}}{T_{S R}-v_{N}}
$$

Replacing in eq. (3)

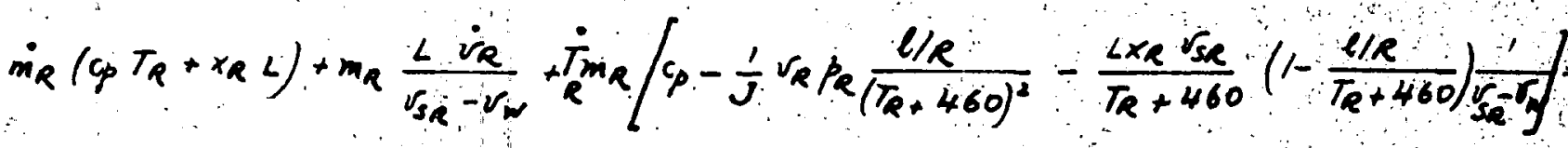

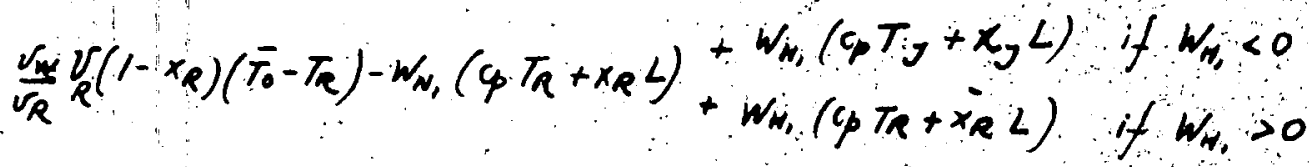

Finaliy

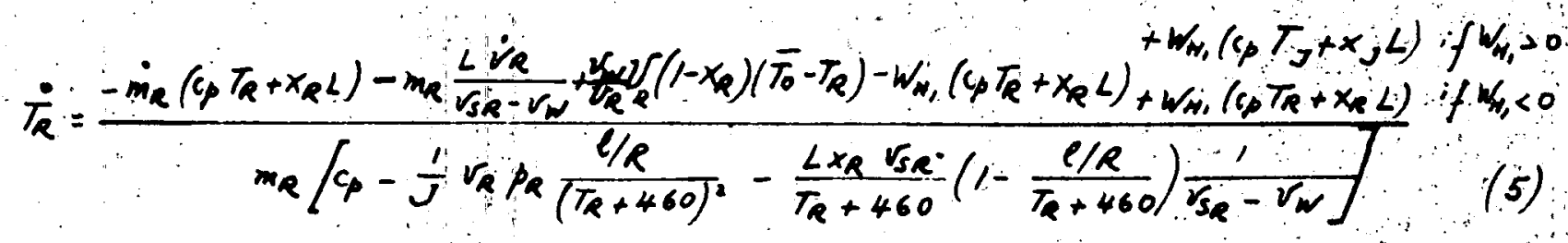

In order to solve the derivatives; the procedure is the following:

Eq. (1) gives $\dot{m}_{R}$, where $W_{N}$, and $W_{N}$, should be previously determined from the equations for the fluid flow resistances.

Eq. (2) gives it :

Using ime dnd $\dot{v}_{R}, e q(s)$ gives $\dot{T}_{R}$

Using $\dot{v}_{R}$ and $\dot{t}_{R}$, eq (*) gives $\dot{x}_{R}$

Using $\dot{T}_{R}$, eq. (3) gives $p_{R}$

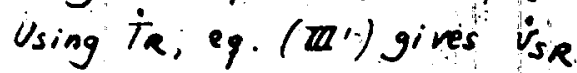

-

If it is desired to use the same routine for getting the initial pordmeters, only the following derivatives can be calculated

BLOW-DOWN ACCIDENT

6000416 


$$
\begin{aligned}
& m_{R}=-W_{N_{1}}+W_{H_{1}}
\end{aligned}
$$

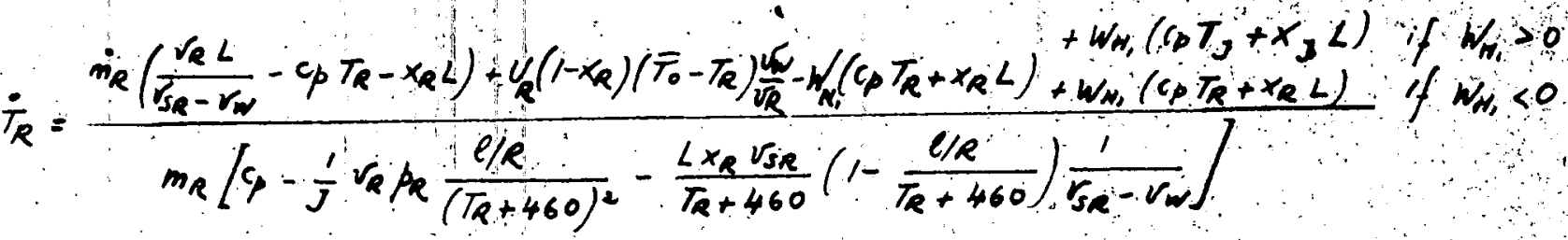

$$
\begin{aligned}
& \dot{x}_{R}=\frac{-V_{R} \frac{\dot{m}_{R}}{m_{R}}}{r_{S R}-v_{W}}-\frac{x_{R} V_{S R}}{V_{S R}-V_{W}}\left(1-\frac{l / R}{T_{R}+460}\right) \frac{\dot{T}_{R}}{T_{R}+460}
\end{aligned}
$$

The other parameters; at the ind of the time interval $\Delta t$, can be calculated as before using the above mentioned parameters at the end of the time inter vil; ice.

$$
\begin{aligned}
& m_{R}(\Delta t)=m_{R_{0}}+\dot{m}_{R} \Delta t \\
& T_{R}(\Delta t)=T_{R_{0}}+\dot{r}_{R} \Delta t \\
& x_{R}(\Delta t)=x_{R_{0}}+\dot{x}_{R} \Delta t
\end{aligned}
$$

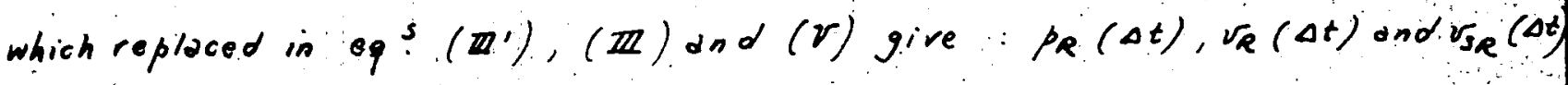
BLOW-DOWN ACCIDENT

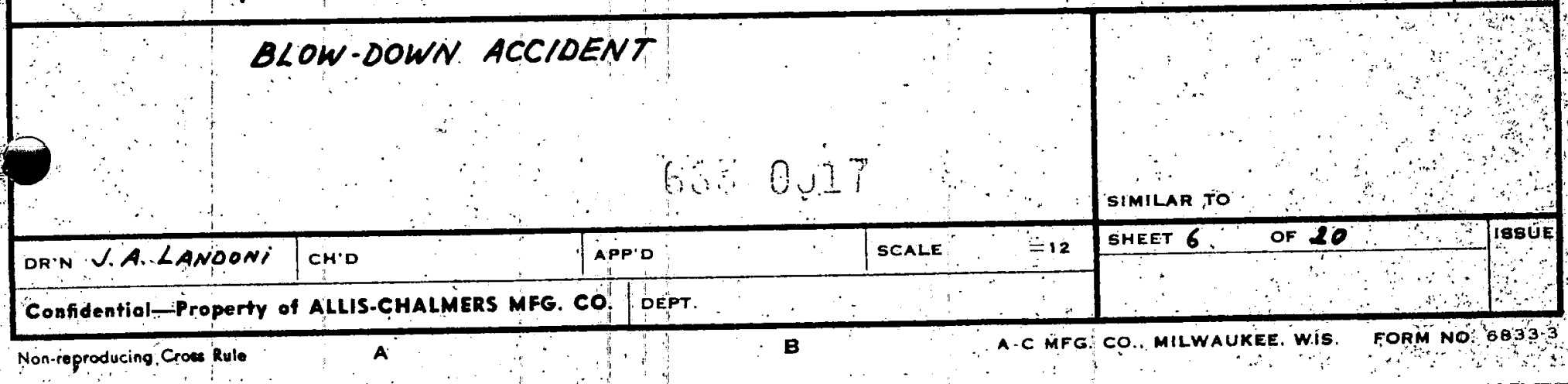

Non-reproducing Cross Rule

A

B

A-C MFG CO.. MILWAUKEE. WiS. 
Ruptured Pipe.

STEAM GENERATOR SIDE LL

i. Pressure Balance

$$
p_{G}-\frac{w_{L}^{2}}{A_{L}^{2}} \frac{r_{G}}{2 g}-p_{2}=k_{2} w_{L}^{2} \bar{r}_{L}
$$

2. Specific volume of the exit:

$$
r_{2}:=\left(1-x_{2}\right) \cdot r_{w_{1}}+x_{4} v_{s_{2}}
$$

i. Specific volume in the resistance:

$$
\bar{v}_{2} \approx-\frac{1}{2}\left(v_{6}+v_{6}\right)
$$

4. Specific volume of the saturated steam at the exit:

$$
V_{S_{L}}=\left(\frac{R}{\mu}\right) \frac{T_{L}+460}{p_{0}} e^{\frac{\ell / R}{T_{2}+460}}
$$

(IV)

5. Pressure of the exit:
\[ \quad p_{2}=p_{0} e \]
$\vdots \quad$ Continuity equation:

$$
V_{L}=\frac{A_{L}}{V_{L}} u_{c_{L}}
$$

(vi)

7. Energy balance:

$$
c_{c} T_{c}+x_{c} L=c_{1} T_{L}+x_{c} L+\frac{\mu_{c}^{2}}{2 g !}
$$

8. Sonic velocity of the exit

The sonic velocity is given by

$$
\begin{aligned}
& u_{c}=\sqrt{g} \gamma\left(\frac{\partial \rho}{\partial \rho}\right)_{s} \\
& \text { As } \frac{\partial p}{\partial \rho}=\frac{\partial p}{\partial v} \cdot \frac{d v}{\partial \rho} \\
& \text { and } \rho=\frac{i}{v} \quad, \\
& d \rho=-\frac{1}{v^{2}} d v \quad \frac{d v}{d \rho}=-v^{2} \\
& \frac{\partial \rho}{\partial \rho}=-v^{2} \frac{\partial p}{\partial v}
\end{aligned}
$$

(vii)$$
\text { BLOW-DOWN ACCIDENT }
$$

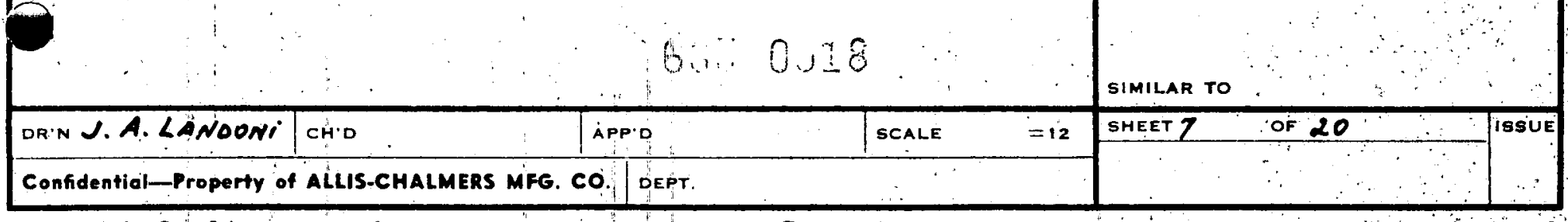

Non-Pproducing Crow Rule

$\mathbf{B}$

A.C MFG. CO., MILWAUKEe, wis. FORM Nö-6B̈3-3. 
As an adiabatic process

$$
d_{i}=d Q+\frac{1}{J} v d p=\frac{1}{J} v d p
$$

being $\quad d i=c d T+L d x$

Differentiating the clapeyron's law

$$
d p=p \frac{e / R}{(T+460)^{2}} d T
$$

and $\quad d T=\frac{(T+460)^{2}}{l / R} \frac{d p}{P}$

Differentioting the expression for the specific volume:

$$
\begin{aligned}
& v=(1-x) v_{\text {ww }}+x v_{s} \\
& d v=-v_{w} d x+v_{s} d x+x d v_{s}=\left(v_{s}-v_{w}\right) d x+x d v_{s}
\end{aligned}
$$

and

$$
d x=\frac{d v}{v_{s}-v_{w}}-\frac{x d v_{s}}{v_{s}-v_{w}}
$$

From

$$
\begin{aligned}
& r_{s}=\left(\frac{R}{\mu}\right) \frac{T+460}{p} \\
& \frac{d v_{s}}{r_{s}}=\frac{d T}{T+460}-\frac{d p}{p}
\end{aligned}
$$

and

$$
d x=\frac{d v}{r_{s}-v_{w}}-\frac{x v_{s}}{r_{s}-v_{w}}\left(\frac{d T}{T+460}-\frac{d p}{p_{p}}\right) .
$$

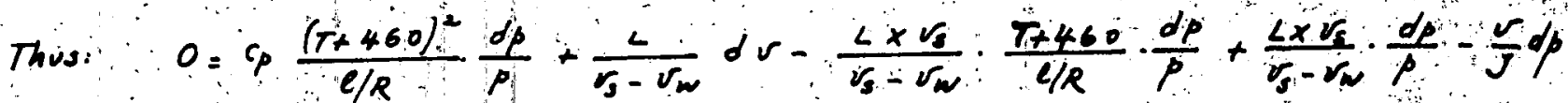

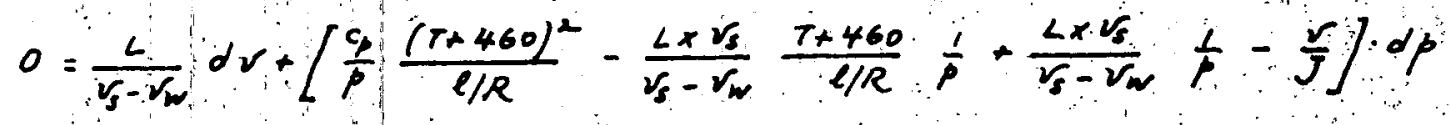

and

$$
\begin{aligned}
& \frac{d p}{d v}=-\frac{\frac{L}{V_{s}-V_{w}}}{\frac{c}{P} \frac{(T+460)^{2}}{l / R}+\frac{L \times V_{s}}{\left(V_{s}-V_{w}\right) P}\left(1-\frac{T+460}{P T R}\right)-\frac{V}{J}} \\
& \frac{\partial p}{\partial \rho}=v^{2} \frac{p \frac{L}{v_{s}-V_{w}}}{c_{p} \frac{(T+460)^{2}}{l / R}+\frac{L \times V_{s}}{r_{s}-V_{v}}\left(1-\frac{T+460}{l / R}\right)-\frac{R V}{J}}
\end{aligned}
$$

The sonic velocity is given by

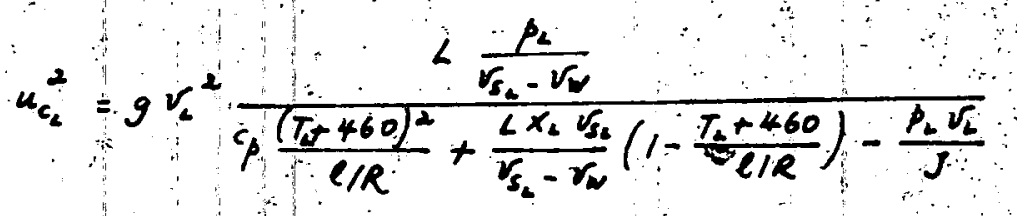

\begin{tabular}{|l|l|l|l|l|l}
\hline DRN VIA. LANOONI & CHD & APPD & SCALE & $=12$ \\
\hline
\end{tabular}


Hor Leg Pipes: $H_{1}, H_{2}, I$ and Joint $J$

1. Pressure Balance Pressurizer -Steam Generator

$$
\begin{aligned}
p_{P}-p_{G}=k_{I} w_{I}^{2} v_{P} & +k_{W_{2}} w_{H_{2}}^{2} v_{1} ; \quad ; p_{J}>p_{G} \text { and } w_{w_{2}}>0 \\
& -k_{H_{2}} w_{H_{2}}^{2} v_{G} ; p_{J}<p_{G} \text { and } w_{H_{2}}<0
\end{aligned}
$$

Pressurizer -Reactor Vessel

$+k_{N_{1}} W_{N_{1}}^{2} r_{J} ; \beta_{J}>\beta_{R}$ and $W_{N_{0}}>0$

$$
-k_{A_{1}} w_{M_{1}}^{2} v_{R} \quad ; \beta_{J}<\beta_{R} \text { and } w_{A_{1}}<0
$$

2. Continuity equation:

$$
W_{1}=w_{H_{1}}+\dot{w}_{H_{2}}
$$

3. Energy balance:

$$
\begin{aligned}
& W_{H_{2}}\left(c_{p} J+x, L\right)+W_{H_{1}}\left(c_{p} \tau+x_{\nu} L\right) ; \beta_{j} \nu p_{6} ; p_{j}>p_{e} \\
& \left.W_{I}\left(c_{p} T_{p}+x_{p} L\right)=W_{W_{2}}\left(c_{p} T_{G}+x_{G} L\right)+w_{M_{1}}\left(c_{p} T_{R}+x_{R} L\right) ; p_{j}<p_{G}\right) p_{j}<p_{R}
\end{aligned}
$$

(ID)

4. Saturation conditions at the joint:

$$
\begin{gathered}
p_{p}-k_{I} w_{x}^{2} r_{p}=p_{J}=p_{0} e^{\frac{l / R}{J+460}} \\
r_{s j}=\left(\frac{R}{\mu}\right) \frac{\tau+460}{p_{0}} e^{\frac{\ell / R}{J+460}}
\end{gathered}
$$

5. Specific volume of the joint:

$$
\left.v_{5}=\left(1-x_{j}\right) v_{w}+x_{j} v_{2}\right)
$$

(vii)

BLOW-DOWN ACCIDENT

$$
\text { Gu: } 0,20
$$

DRIN STA. LANDON

CHAD

APP'D

SIMILAR TO

Confidential-Property of ALLIS-CHALMERS MFG. CO DEPT.

SCALE

$=12$

SHEET 9 . 20

love

Non-roproducing Crone Rule.

B

A.C -MFG CO.. MILWAUKEE, WIS.

T RM NO 68333

$\therefore \cdots$ 
Heat Balance:

$$
F \cdot \dot{\bar{T}}_{0}=c_{1} t^{-0.2}+c_{2} e^{-\lambda t}-U_{R}\left(1-x_{R}\right)\left(\bar{T}_{0}-T_{R}\right) \frac{\psi_{w}}{v_{R}}
$$


Reactor Vessel Capacitance: $R$

$$
\begin{aligned}
& \dot{m}_{R}=-w_{N_{1}}+w_{N_{1}} \\
& \text { (i) }
\end{aligned}
$$

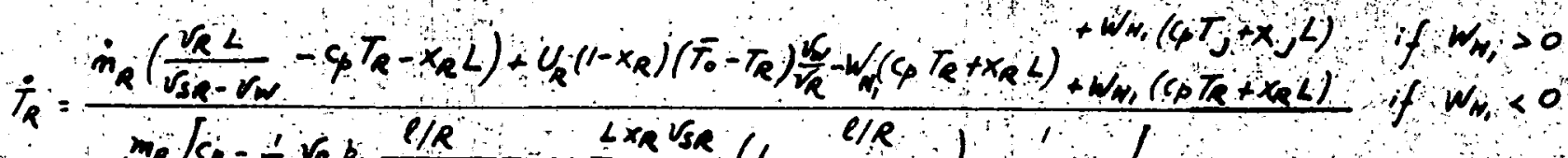

$$
\begin{aligned}
& m_{R}\left[c_{p}-\frac{1}{J} v_{R} p_{R} \frac{l / R}{\left(T_{R}+460\right)^{2}}-\frac{L x_{R} v_{s R}}{T_{R}+460}\left(1-\frac{l / R}{T_{R}+460}\right) \frac{1}{v_{S_{R}}-v_{n}}\right] \\
& x_{R}=\frac{-v_{R} \frac{m_{R}}{m R}}{r_{S R}-v_{N}}-\frac{x_{R} v_{S R}}{V_{S_{R}}-v_{W}}\left(1-\frac{l / R}{T_{R}+460}\right) \frac{T_{R}}{T_{R}+460} \\
& \text { (3) } \\
& \dot{v}_{R}=-v_{R} \frac{\dot{m}_{R}}{m_{R}} \\
& \dot{r}_{S R}=v_{S R}\left(1-\frac{e / R}{T_{R}+460}\right) \frac{T_{R}}{T_{R}+460} \\
& \dot{p}_{R}=p_{R} \frac{e / R}{\left(T_{R}+460\right)^{2}} \dot{T_{R}}
\end{aligned}
$$

$$
6, \cdots 0,22
$$

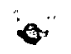

$\omega$

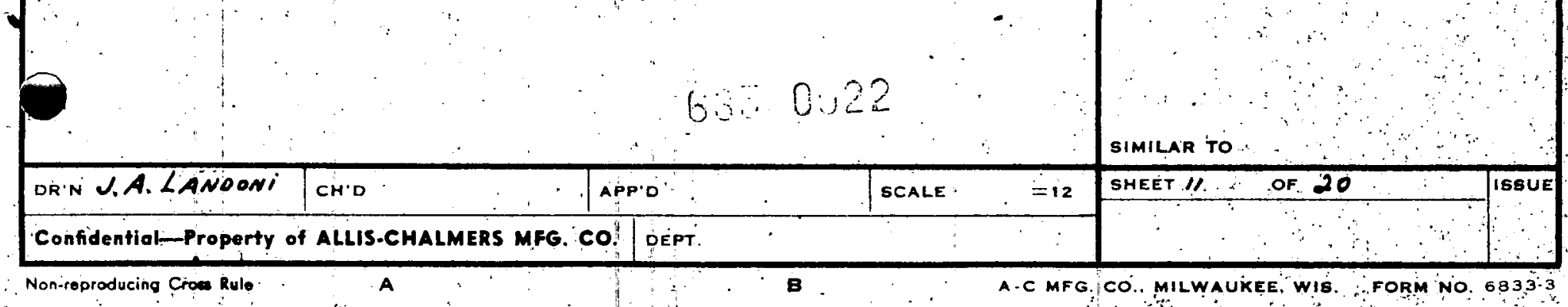

Non-roproducing Croes Rule

$\mathbf{B}$

A-C MFG, CO.. MILWAUKEE, WIS. CFORM NO $6833 \mathrm{3}$ 
$m_{p}=-W_{x}$

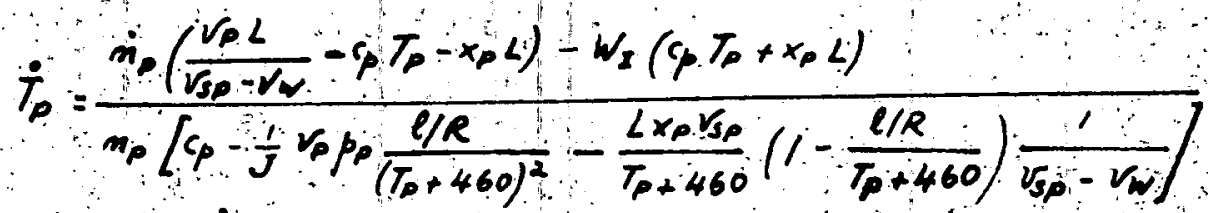

$\dot{x}_{p}=\frac{-v_{p} \frac{m_{p}}{m p}}{v_{s p}-v_{w}}-\frac{x_{p} v_{s p}}{V_{s p}-v_{w}}\left(1-\frac{l / R}{T_{P}+460}\right) \frac{\dot{T}_{p}}{T_{p}+460}$

$\dot{v}_{p}=-v_{p} \cdot \frac{\dot{m}_{p}}{m_{p}}$

$v_{s p}=v_{s p}\left(1-\frac{l / R}{T_{P}+460}\right) \frac{\dot{T_{p}}}{T_{p}+460}$

$\dot{p}_{p}=p_{p} \frac{l / R}{\left(T_{p}+460\right)^{2}} \dot{T}_{p}$ 
STEAM GENERATOR: $G$

$$
\dot{r}_{c}=w_{H_{2}}-w_{L}
$$

(i)

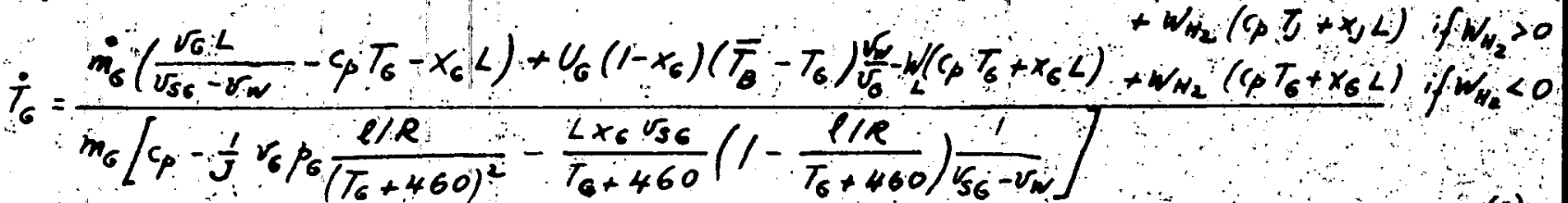

$\dot{x}_{G}=\frac{-v_{G} \frac{\dot{m}_{G}}{v_{S_{G}}-v_{W}}}{v_{W}}-\frac{x_{\sigma} v_{S G}}{v_{S G}-v_{W}}\left(1-\frac{\rho / R}{T_{G}+460}\right) \frac{\dot{T}_{G}}{T_{G}+460}$

$\ddot{v}_{G}=-v_{G} \frac{\dot{m}_{G}}{\dot{m}_{G}}$

$\dot{v}_{s 6}=v_{56}\left(1-\frac{l / R}{T_{6}+460}\right) \frac{\dot{T}_{6}}{T_{6}+460}$

$\dot{p}_{6}=p_{6} \frac{\ell / R}{\left(T_{6}+460\right)^{2}} \dot{T}_{6}$

(6)

$\boldsymbol{\omega}$

BLOW-DOWN ACCIOENT

$b \quad 6 \quad 60$ by

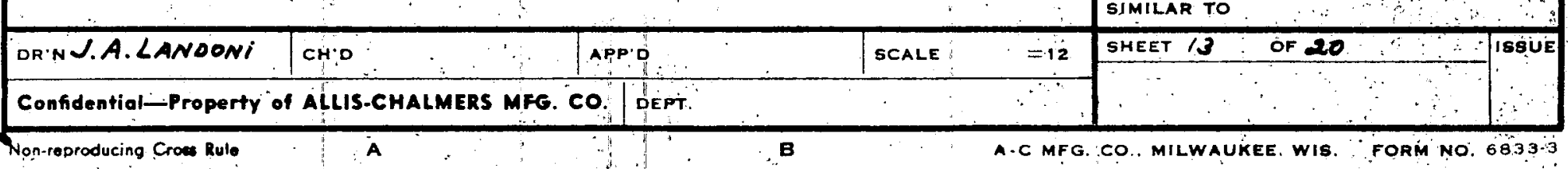

$\omega$ 
Steam Generator Side of the Ruptured Pipe: L

$$
\begin{aligned}
& \beta_{G}-\frac{W_{2}^{2}}{A_{L}^{2}} \cdot \frac{v_{G}}{2 g}-\beta_{2}=k_{2} W_{L}^{2} \bar{v}_{2} \\
& v_{2}=\left(1-x_{2}\right) v_{w 1}+x_{4} r_{s 2} \\
& \bar{r}_{2}=\frac{1}{2}\left(r_{6}+r_{2}\right) \\
& v_{S_{2}}=\left(\frac{R}{\mu}\right) \frac{T_{L}+460}{p_{0}} e^{\frac{l / R}{T_{L}+460}} \\
& p_{L}=\beta_{0} e^{-\frac{l / R}{T_{L}+460}} \\
& W_{L}=\frac{A_{L}}{V_{L}} u_{c_{2}} \\
& c_{p} T_{\sigma}+x_{G} L=c_{p} T_{L}+x_{L} L+\frac{u_{c L}^{2}}{2 g J}
\end{aligned}
$$

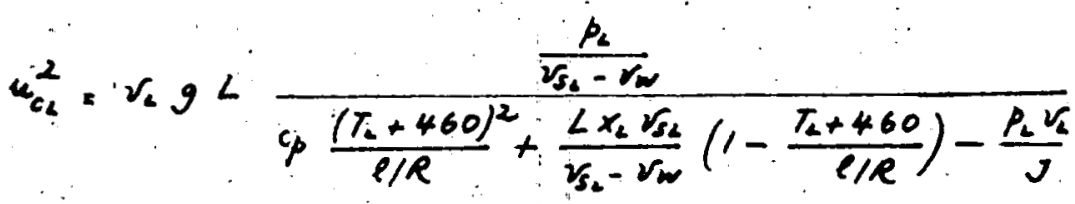

(1)

BLOW-DOWN ACCIDENT

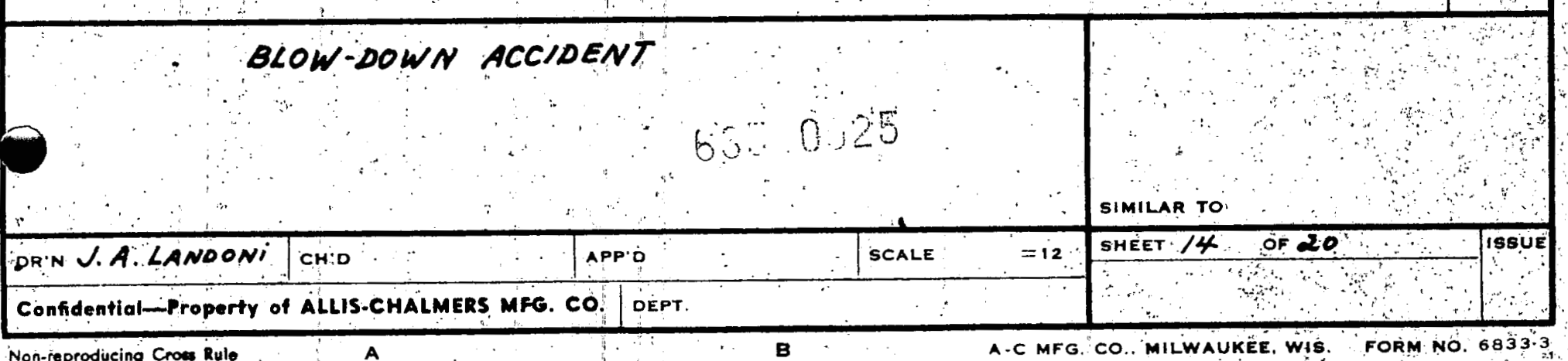

Non-reproducing Cross Rule

$\mathbf{B}$

A.C MFG CO.. MILWAUKEE, WIS.

$\therefore \because-1$ 


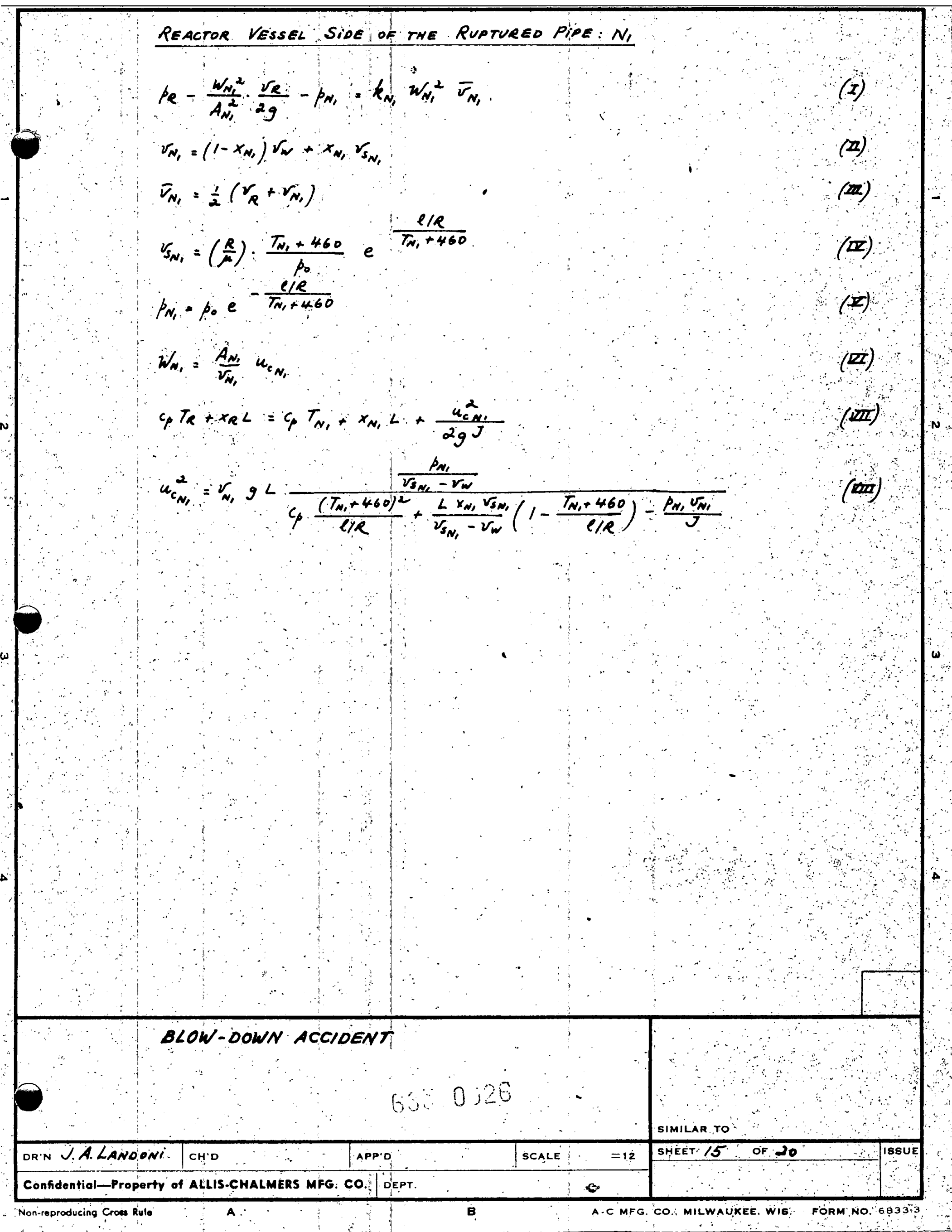




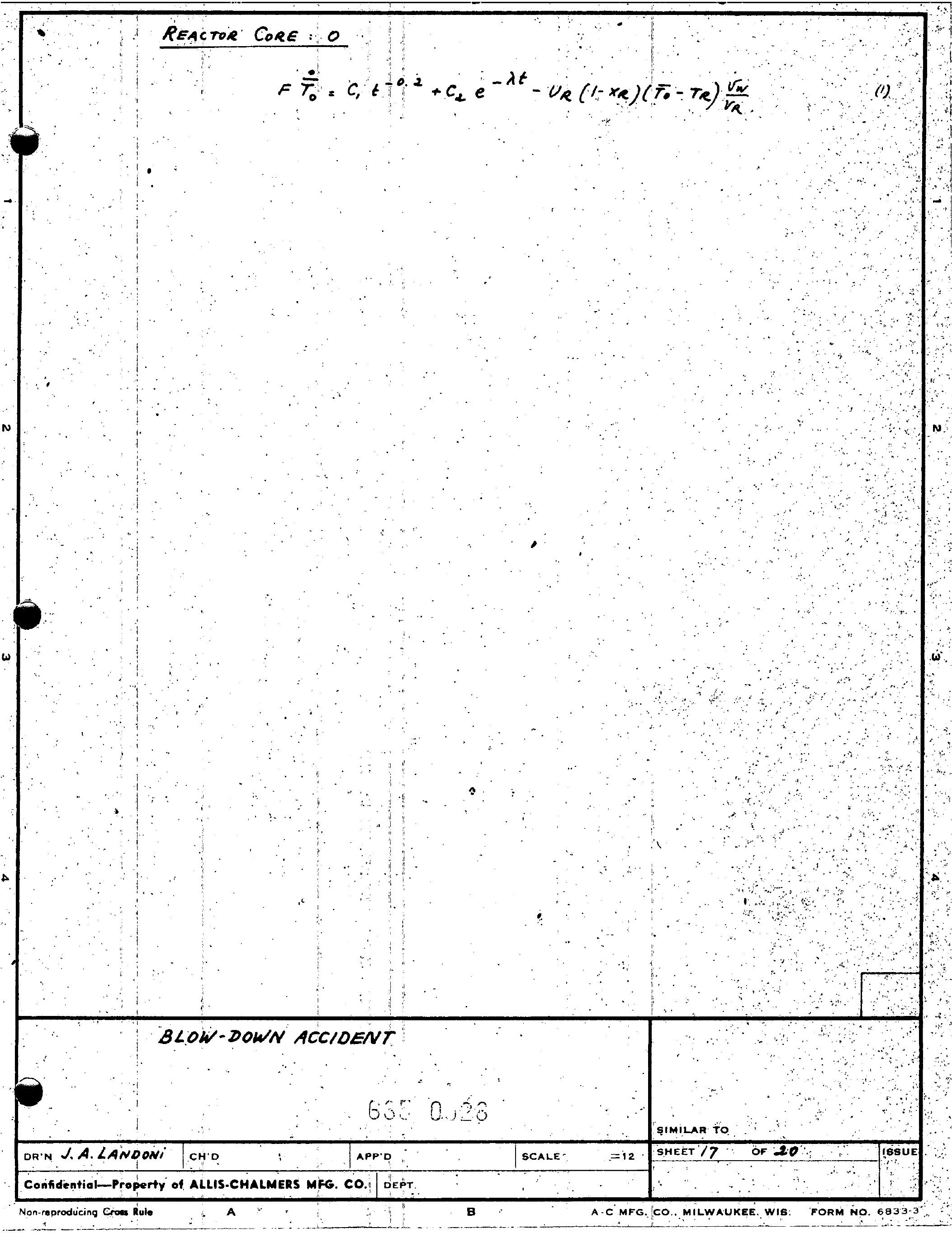


$m_{R}(t=0)=18,80016$

$x_{R}(t=0)=0$

$T_{R}(t=0)=285^{\circ} \mathrm{F}$

$\nu_{R}=3 / 3 f^{3}$

$m_{p}(t=0)=2,1134$

$x_{p}(t=0)=0.006,2$

$T_{p}(t=0)=4 i 0.3 \cdot F$

$V_{p}=58 f+{ }^{\circ}$

$m_{\sigma}(t=0)=13,90016$

$x_{G}(t=0)=0$

$T_{6}(t: 0)=285: F$

$r_{6}=232 \mathrm{ft}^{3}$.

$\beta_{J}(t=0)=\beta_{p}(t=0)=4: 24 \cdot 104 \frac{13}{f_{t}^{2}}$

$\overline{T_{0}}(t=0)$ a $423 \cdot \mathrm{F}$

$\therefore \overline{T_{B}}=2.50 \cdot F$

$U_{R}=268 \frac{8 . T U}{\mathrm{sec} \cdot \mathrm{F}}$

$V_{G}=1,086 \cdot \frac{8 T U}{\sec \cdot F}$

$A_{2}=A_{N_{1}}=1.42 f \times 2$

$k_{N_{1}}=1.0 \times 10^{-3} \frac{\mathrm{sec}^{2}}{f+5} \quad \therefore \quad ; k_{2}=0.040 \frac{\mathrm{sec}^{2}}{f+5} \quad ; k_{H_{1}}=k_{N_{2}}=0.030 \frac{\mathrm{sec}^{2}}{f^{5}}$

$\ell_{1} \approx k_{4}$

$c_{p}=1 \frac{B T U}{16 \cdot F}$

$L=890^{\circ} \frac{B T U}{16}$

$r_{10} \approx \frac{1}{60} \frac{7 t^{3}}{16}$

$\frac{R}{\mu}=85.9 \frac{f t}{F}$

$C_{1}=2.28 \times 10^{\mathrm{s}}$ BTU sec -0.8

$\frac{l}{l}=8.833 \%$

$C_{2}=28.8 \cdot 10^{3}, \frac{B r U}{\sec }$

$\gamma_{i}=0.1 \mathrm{sec}^{-}$

$J=778 \cdot \frac{16 f t}{B r V}$

$F: 674 \frac{B T U}{F}$

$g: 32.2 \mathrm{ft} / \mathrm{sec}^{2}$

$p_{0}=1.085 \times 10^{9} \frac{16}{f+2}$

BLOW-DOWN ACCIOENT

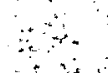

20

$\omega$. 


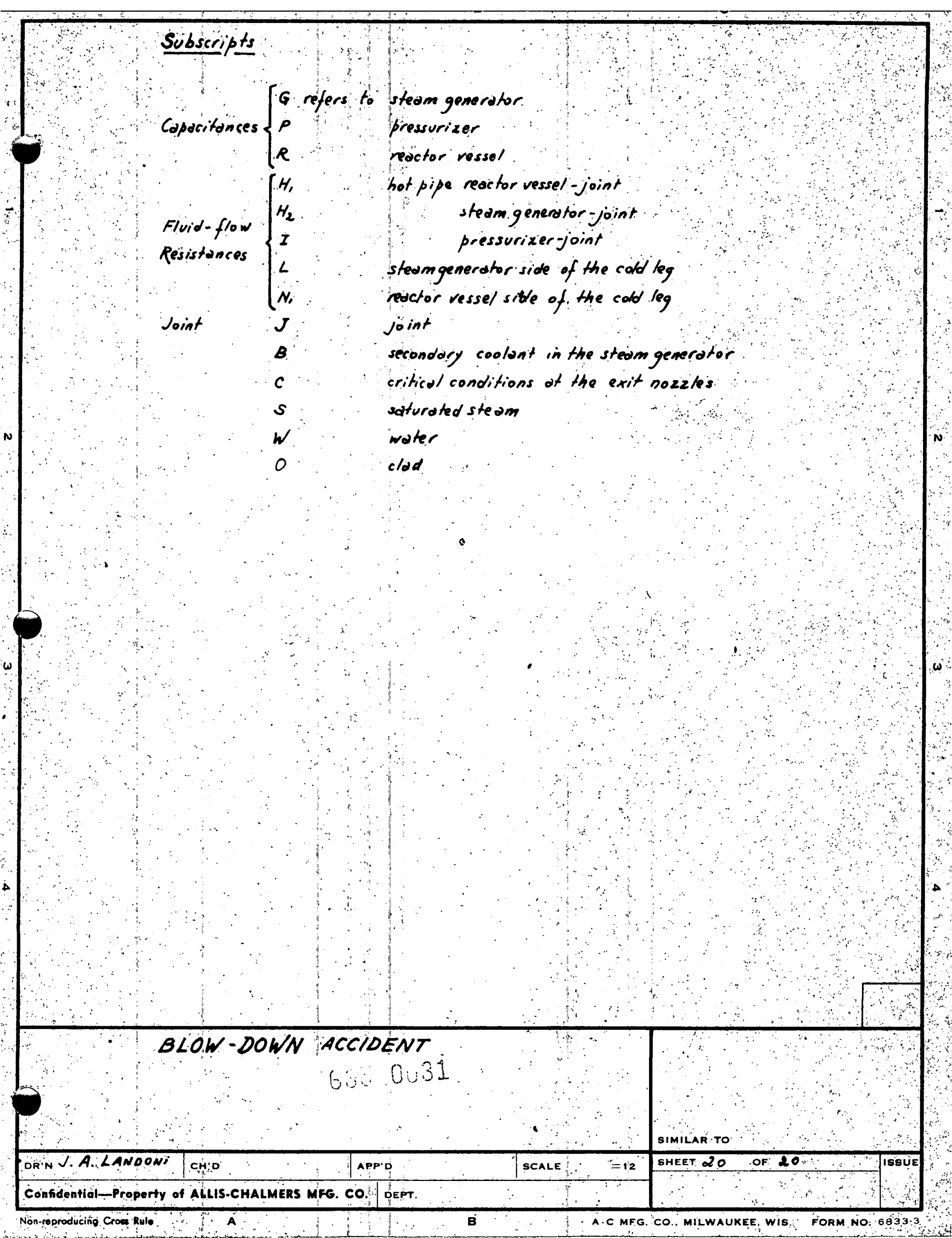


ADDENDUM

REDUCTION IN THE NUMBER OF VARIABLES

REACTOR VESSEL CAPACITANICE:R

Elimination of the specific volume by using the relationship

$$
v_{R}=\frac{v_{R}}{m_{R}}
$$

being $V_{e}$ the capacitance volume, which is constant.

Replacing ir in equations (2) and (3) the remaining set of 5 equations is as follows

$$
\begin{aligned}
& \dot{m}_{R}=-W_{N_{1}}+W_{N_{1}} \\
& \text { (i) }
\end{aligned}
$$

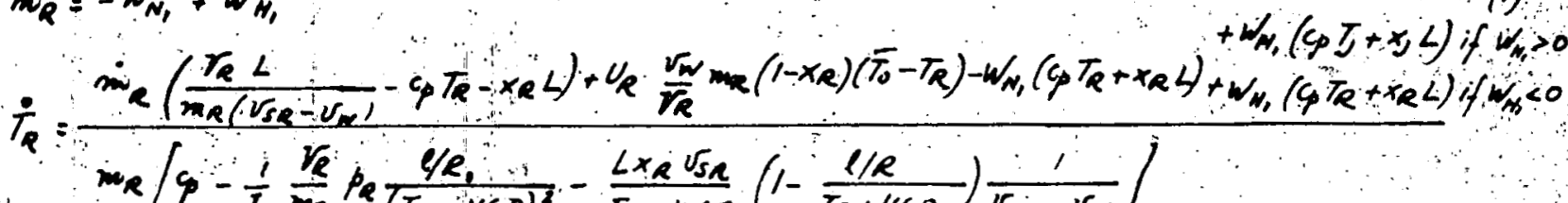

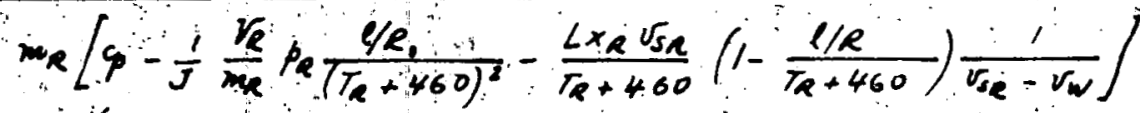

$$
\begin{aligned}
& \dot{x}_{R}=\frac{\frac{V_{R}}{m_{R^{2}}} \dot{m}_{R}}{V_{S R}-V_{N}}-\frac{x_{R} V_{S R}}{V_{S R}-V_{N}}\left(1-\frac{l / R}{T_{R}+460}\right) \frac{\dot{T}_{R}}{T_{R}+460} \\
& \dot{v}_{S R}=v_{S R}\left(1-\frac{\ell R}{T_{R}+460}\right) \frac{T_{R}}{T_{R}+660} \\
& \dot{p}_{R}=\frac{l / R}{\left(T_{R}+460\right)^{2}} \dot{T}_{R}
\end{aligned}
$$

2. Elimination of the variable $\nu_{s R}$ by using the relationship

$\omega$

and

Thins

$$
\begin{aligned}
& v_{R}=\left(1-x_{Q}\right) v_{n}+x_{R} r_{s e} \\
& r_{R}=m_{e} r_{R} \\
& x_{R}\left(v_{s R}-r_{w}\right)=\frac{r_{R}-m_{R} \cdot v_{n}}{x_{R}} \\
& \frac{m_{R} \times R r_{S R}}{V_{S R}-v_{W}}=\frac{r_{R}-\left(1-x_{R}\right) v_{W} m_{R}}{r_{R}-m_{R} v_{w}} m_{R} x_{R}=m_{R} x_{R}\left(1+\frac{x_{R} m_{R} v_{W}}{V_{R}-m_{R} v_{W}}\right)
\end{aligned}
$$

The set of 4 equations is os follows

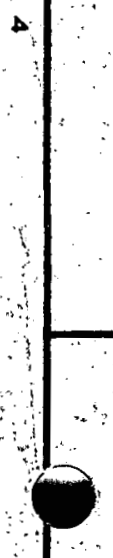

$$
\text { BLOW-DOWN ACCIDENT ADDENDUM }
$$

6300.132

OREN JA. LANDON I

CM P

APP'D

SIMILAR TO

Confidential-Property of ALLIS.CHALMERS MFG.:CO. DEPT.

SCALE

$=12$

SHEET

of 73

isSUE

Non-reproducing Crow Ru ie

B

A.C MFG CO MILWAUKEE WIS FORM NO 6833.3 


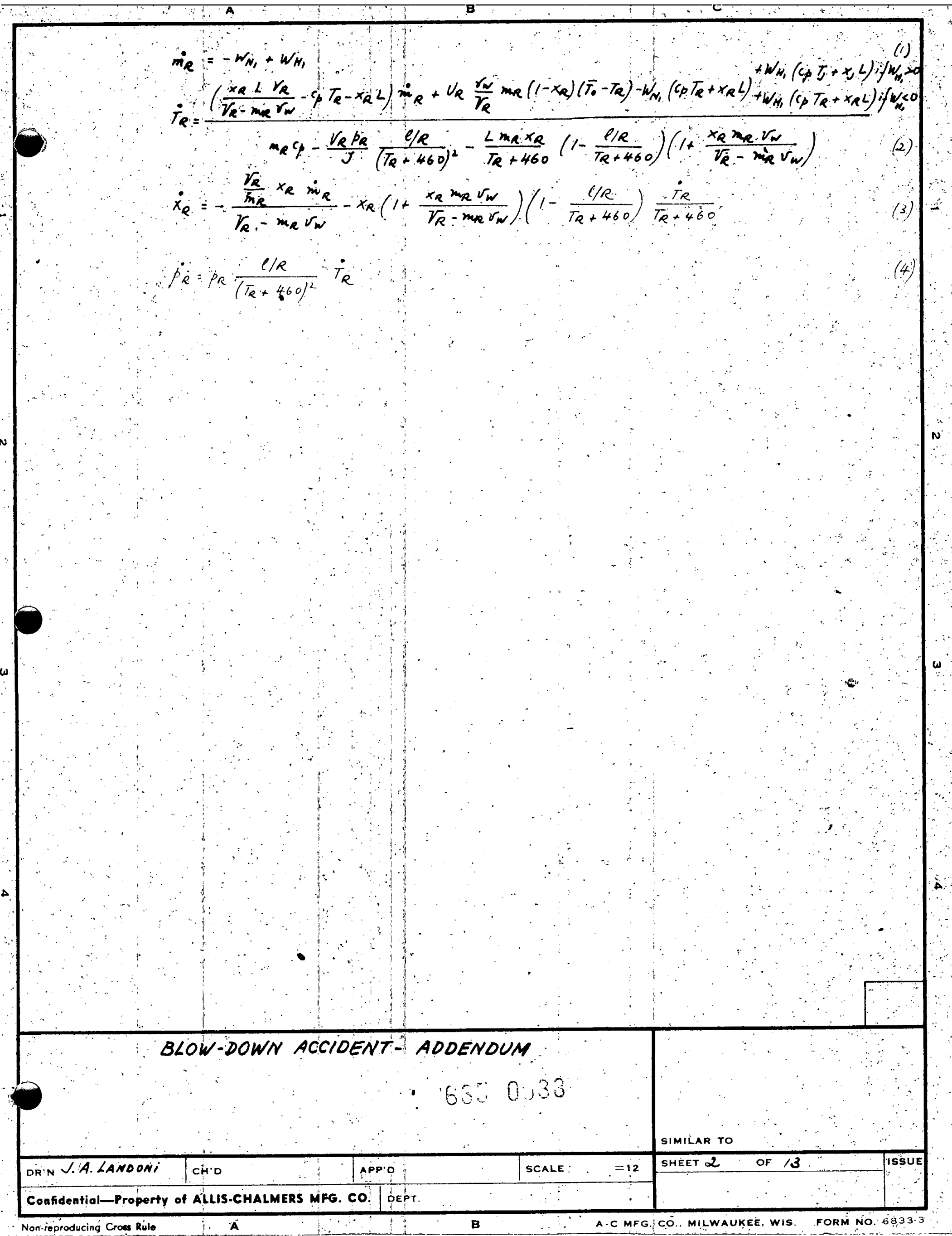


RuPTURED Pipe

STEAM GENERATOR SIDE LL

Elimination of $\vec{r}_{L}$, replacing (II) in (z)

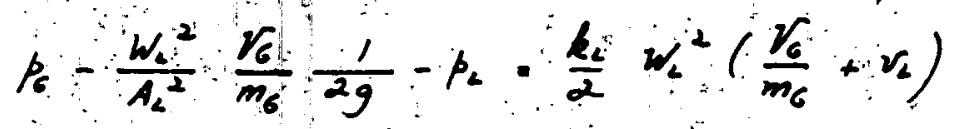

2. Elimination of iss replacing (WT) in (D) and (w)

$$
\begin{aligned}
& v_{i}=\left(1-x_{2}\right) v_{w_{1}}+x_{2}\left(\frac{R}{\mu}\right) \frac{T_{2}+460}{\beta_{2}}
\end{aligned}
$$

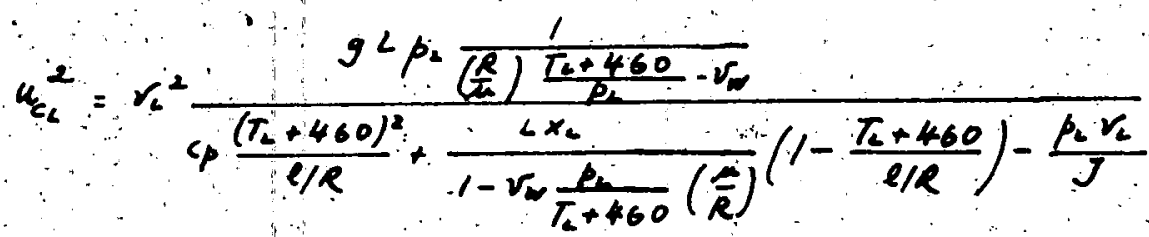

3 Elimination of $u_{c_{2}}$ replacing (6) in (II)

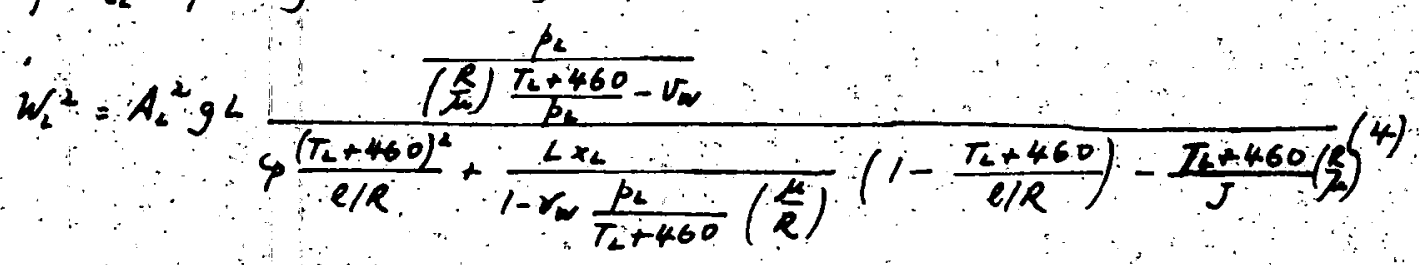

4. Elimination of $v_{i}$ ireplooing $(2)$ in (1):

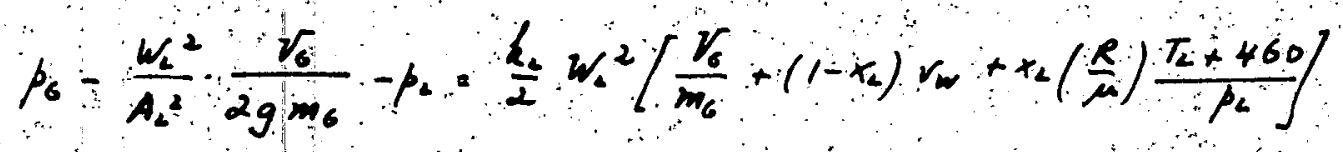

The sot of 4 equations remains as follows

$$
\begin{aligned}
& \beta_{6}-\frac{W_{L}^{2}}{A_{1}^{2}} \frac{V_{6}}{2 g m_{6}}-\beta_{2}=\frac{k_{2}}{2} W_{L}^{2}\left[\frac{V_{6}}{m_{6}}+\left(1-x_{2}\right) v_{N}+x_{2}\left(\frac{R}{\mu}\right) \frac{T_{L}+460}{p_{L}}\right] \\
& \beta_{2} \beta_{0} e^{-\frac{R / R}{T+460}} \\
& W_{L}^{2}=A_{2}^{2} g L \frac{p_{L}}{\left(\frac{R}{2}\right) \frac{1+460}{p_{2}}-v_{w}} \\
& \frac{(\tau+460)^{2}}{l / R}+\frac{L x_{L}}{1-v_{w} \frac{p_{L}}{T_{L}+460}(\hat{R})} \\
& \left(1-\frac{T+460}{l R}\right) \frac{\operatorname{In} 4600\left(\frac{R}{A}\right)}{J} \\
& c_{p} T_{6}+x_{6} L=c_{p} T+x_{L} L+\frac{W_{L}^{2}}{2 g J A_{L}^{2}}\left(\frac{R}{2}\right) \frac{T_{2}+460}{p_{2}}
\end{aligned}
$$

BLOW-DOWN ACCIDENT- ADDENDUM

$60,0,34$

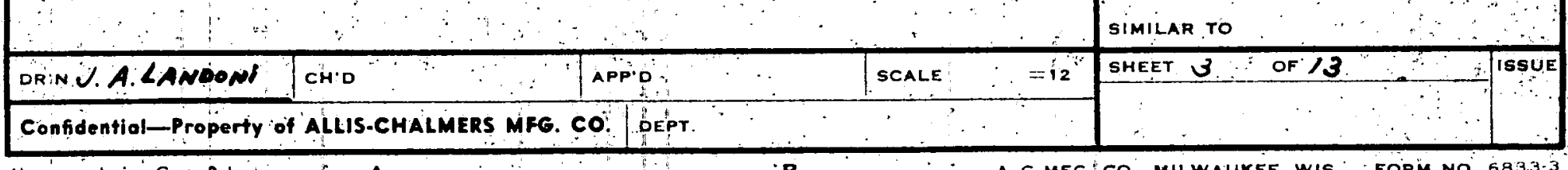

Non'reproducing Crow Rule

$\mathbf{B}$

A-C MFG CO.. MILWAUKEE, WIS.

FORM NO. 683.3 .3 
Hor LeG Pipes H, H H, I \& Join $T$.J

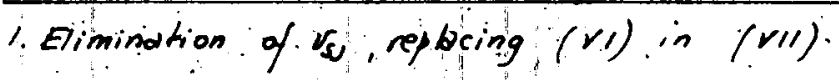

$$
v_{j}=\left(1-x_{j}\right) v_{n}+\frac{x}{p_{j}}\left(\frac{R}{m}\right)(T+460)
$$

2 Elimination of $v_{j}$, replacing $v_{s}$ in (I) and (Z)

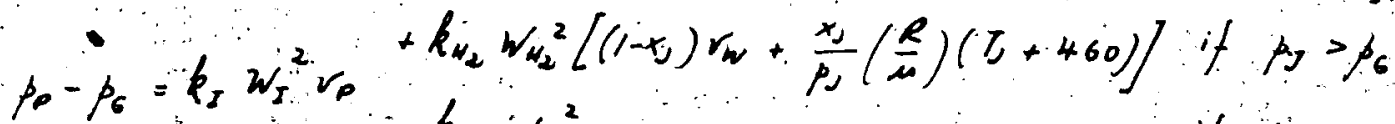

$$
\begin{aligned}
& -k_{\mathrm{H}_{2}} w_{\mathrm{H}_{2}}^{2} r_{G} \quad \text { if } \beta_{6}>p_{\mathrm{J}}
\end{aligned}
$$

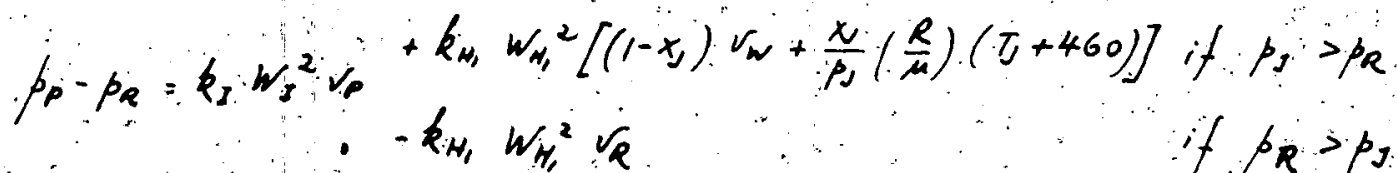

The set of 6 equations can be written as follows

$$
\begin{aligned}
& p_{p}-p_{j}=k_{I} w_{I}^{2} v_{p} / m_{p}
\end{aligned}
$$

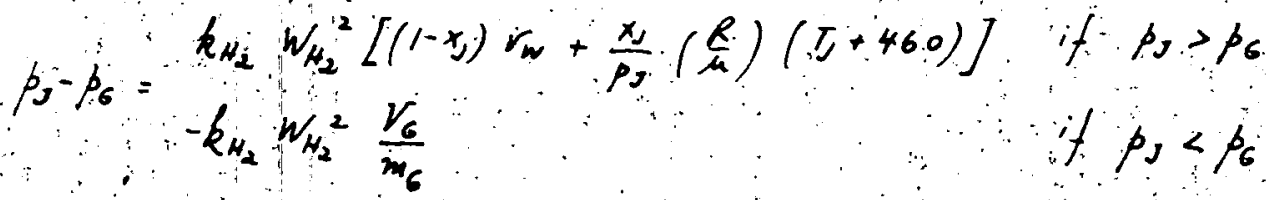

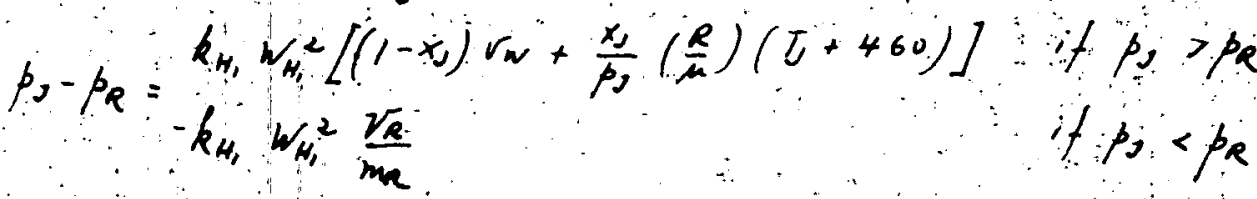

$$
\begin{aligned}
& W_{s}=W_{H_{1}}+W_{H_{2}} \\
& \beta_{S}=\beta_{0} e^{\frac{l / R}{C+460}}
\end{aligned}
$$

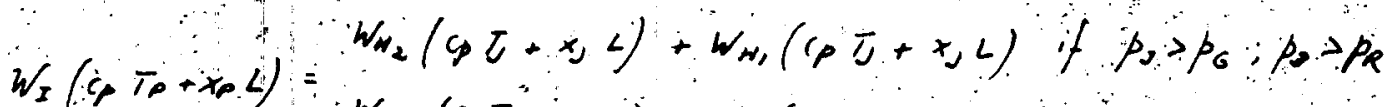

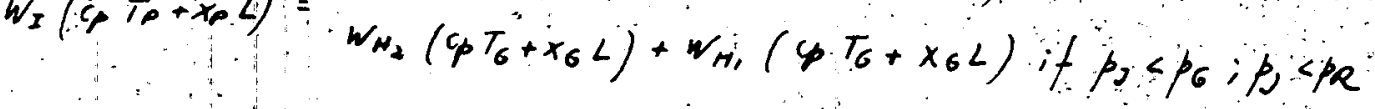

Replocing (4) in (2)

$$
\begin{aligned}
& \beta_{j}-\beta_{6}=k_{N_{2}}\left(W_{1}-W_{N_{1}}\right)^{2}\left[\left(1-x_{J}\right) v_{N}+\frac{x_{1}}{\beta_{1}}\left(\frac{R}{\mu}\right)\left(j_{j}+460\right)\right] \text { if } \beta_{j}>p_{6} \\
& -k_{N_{2}}\left(w_{l}-w_{H_{1}}\right)^{2} \frac{r_{6}}{m_{6}} \quad \cdots p_{J}<p_{6}
\end{aligned}
$$

If $p_{0}>p_{6}$ in (2) ond $p_{0}>p_{R}$ in (a), then, dividing (2) by (3)

$$
\frac{p_{s}-p_{C}}{p_{j}-p_{R}}=\frac{k_{N_{2}}}{k_{H_{1}}}\left(\frac{W_{2}-W_{H_{1}}}{W_{H_{1}}}\right)^{2}
$$

BLOW-DOWN ACCIOENT-ADDENDUM

(6) 


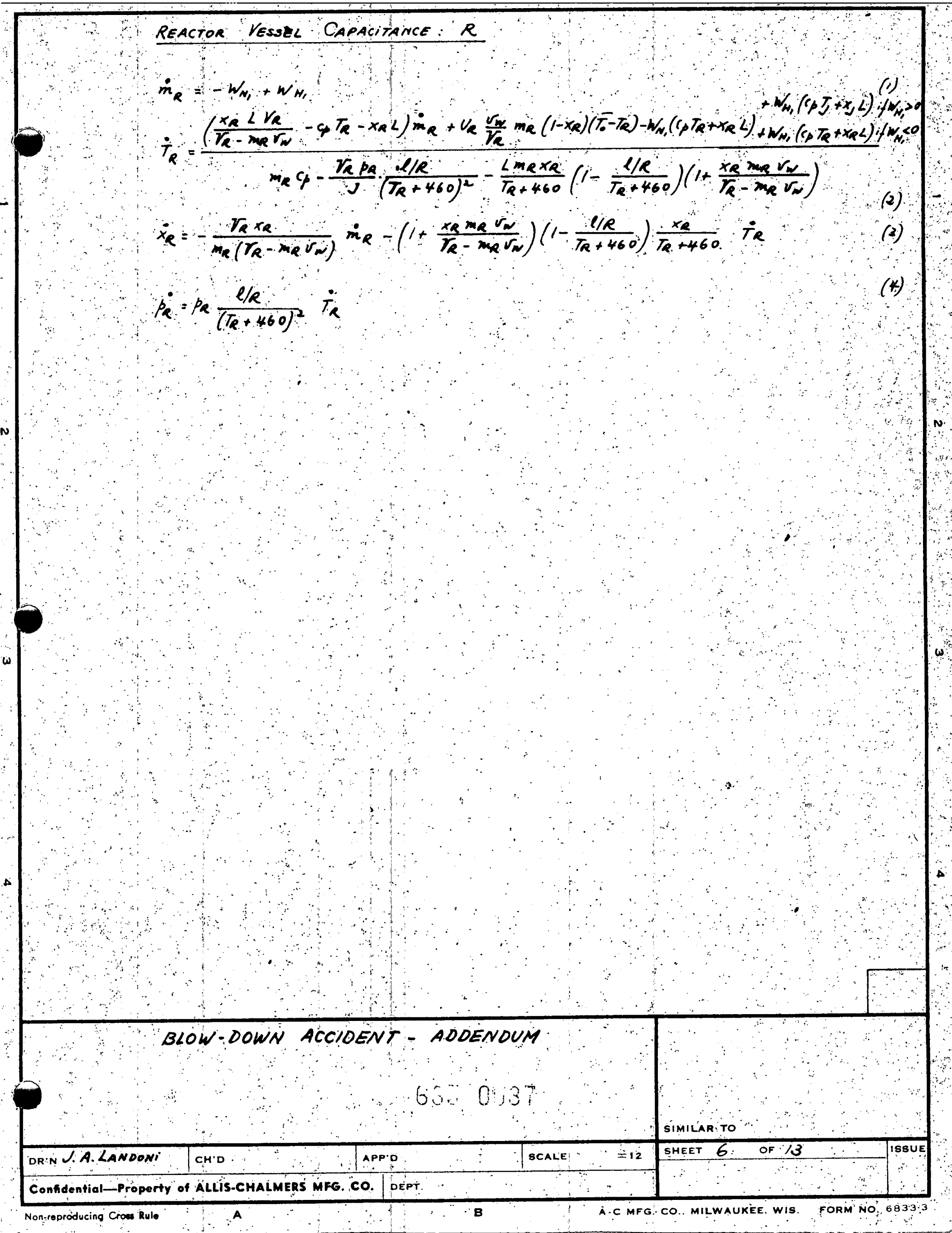




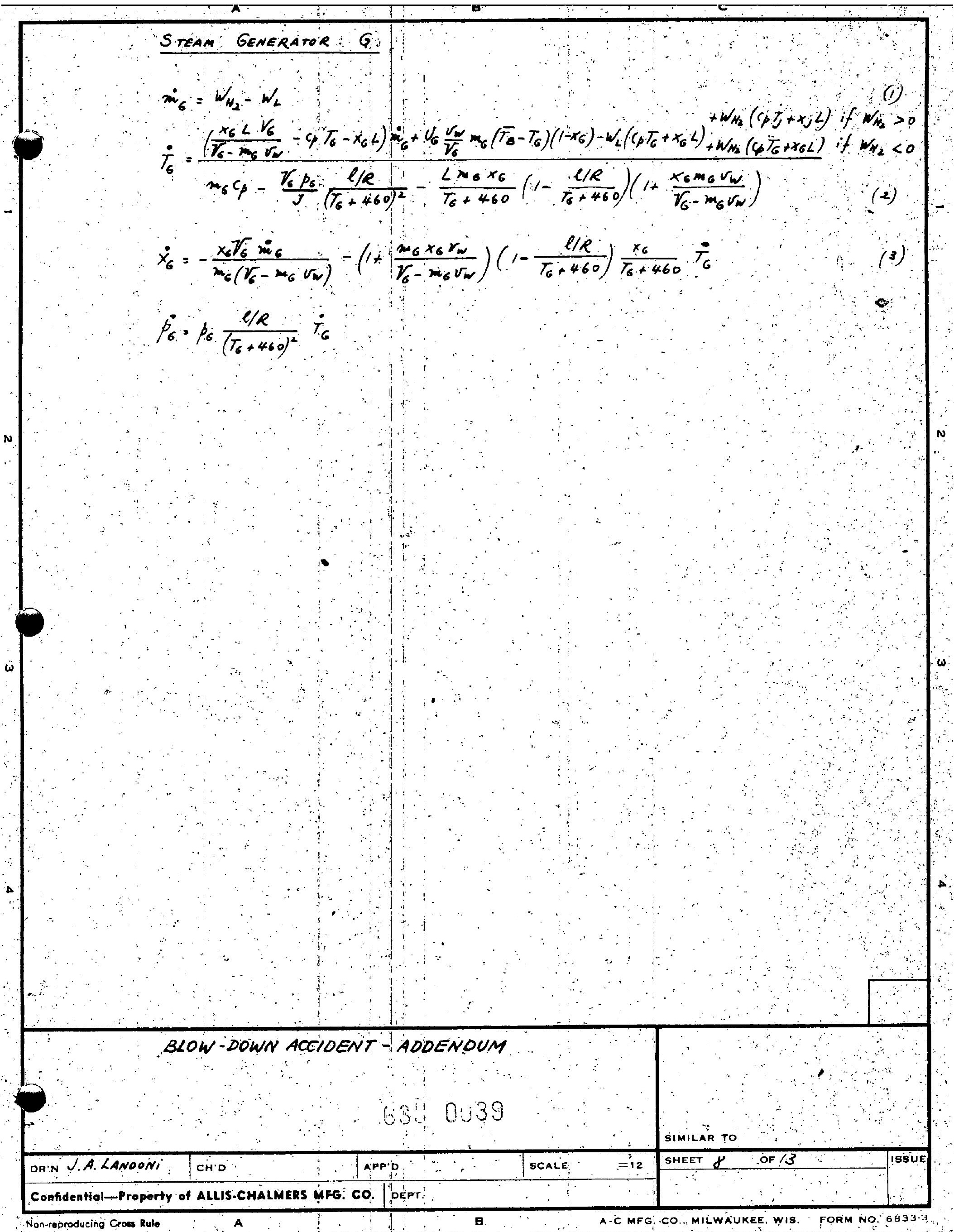


$\left.p_{G}-\frac{N_{L}^{2}}{A_{4}^{2}} \cdot \frac{V_{G}}{2 g m_{6}}-p_{L}+\frac{k_{L}}{2} w_{L}^{2} / \frac{V_{G}}{m_{G}}+\left(1-x_{2}\right) v_{w}+x_{L}\left(\frac{R}{M}\right) \frac{T_{L}+460}{\beta_{L}}\right)$

$p_{2}=p_{0} e^{-\frac{\ell / R}{T_{2}+460}}$

$W_{1}^{2}=A_{1}^{2} g^{2}$

$$
\frac{\frac{p_{L}}{\left(\frac{R}{R}\right) \frac{T_{1}+460}{p_{2}}-\gamma_{2}}}{c_{p} \frac{\left(T_{L}+460\right)^{2}}{l / R}+\frac{L x_{L}}{1-\gamma_{12} \frac{p_{L}}{T_{L}+460}\left(\frac{\hat{R}}{R}\right)}\left(1-\frac{T_{L}+460}{l / R}\right)-\frac{T_{L}+460}{J}\left(\frac{R}{\mu}\right)}
$$

$c_{p} T_{6}+x_{6} L c_{p} T_{2}+x_{c} L+\left(\frac{p}{N_{L}}\right) \frac{T_{c}+460}{2 J}$

$$
\begin{aligned}
& \frac{L}{\left(\frac{R}{A}\right)^{\frac{T+}{2}+460}} \frac{D_{L}}{L}
\end{aligned}
$$

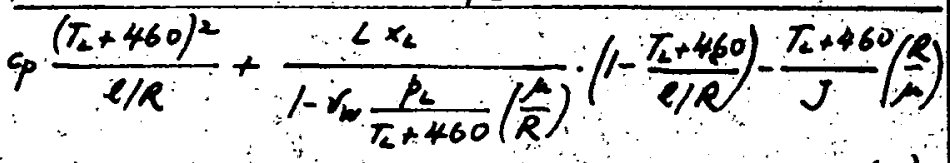

In order to salve this set of equations by trial-and-error the following procodure is suggested 1: Acsime $p_{2}$ in (2) and get $T_{4}$

2. Insert $p_{2}$ dind $T_{2}$ in (4) and get $x_{2}$

3. Insert $p_{2}$, $x_{2}$ and $T_{2}$ in $(3)$ and get $w_{2}$

4. Insert $F_{L}, x_{2}$ and $W_{L}$ in (1) and $p_{2}$ in ond side and get $p_{2}^{\prime}$ in the other side

5. As $p_{2}^{\prime} \neq p_{2}$ the some process is initidted with $\frac{1}{2}\left(\beta_{2}+p_{2}^{\prime}\right)$

6. If $\Delta p_{2}$ is the toterdble error in $\beta_{2}$, when $\left|p_{L}-p_{2}^{\prime}\right|<\Delta p_{2}$ tho volue of $\beta_{2}$ is docepted together with the other porameters. 


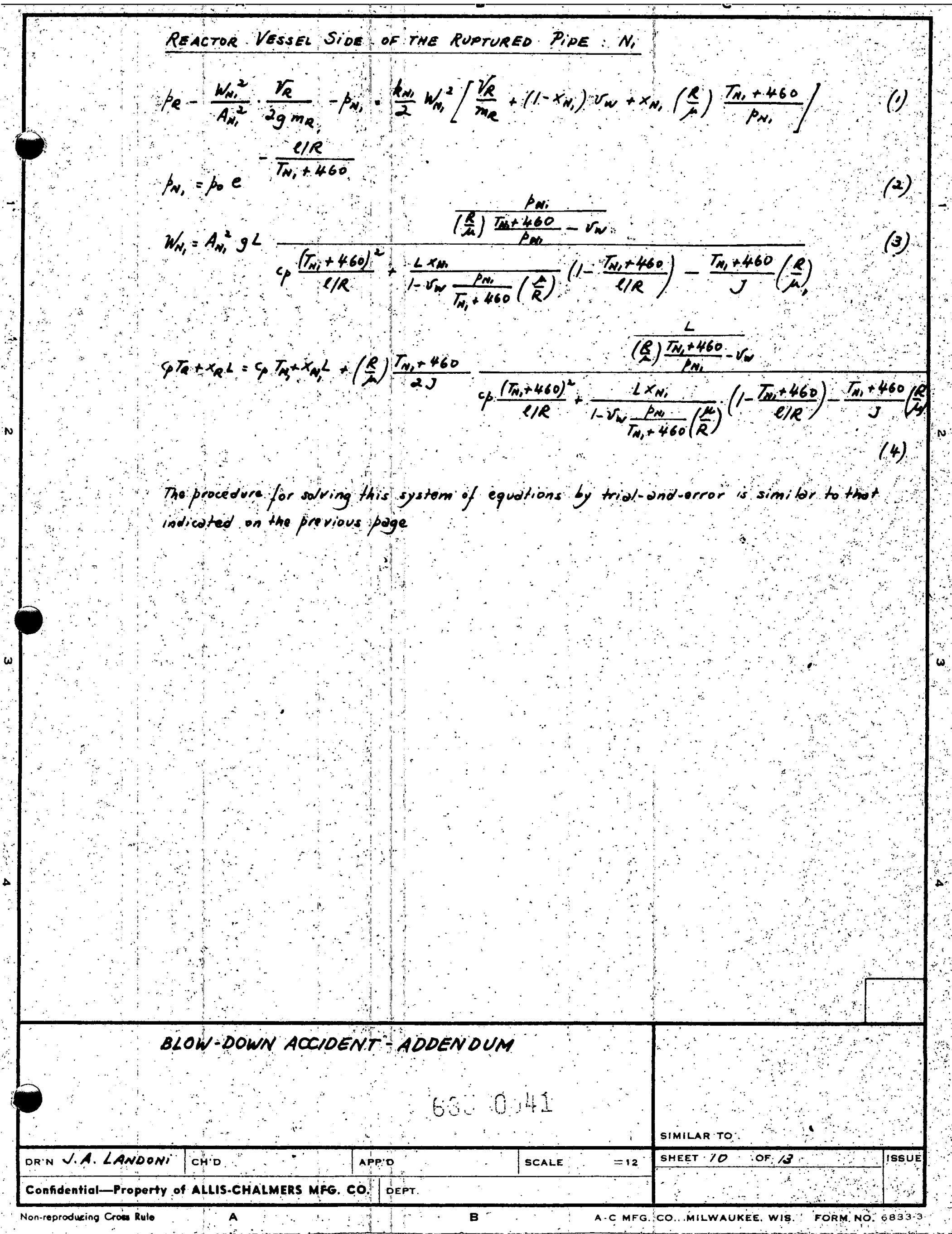




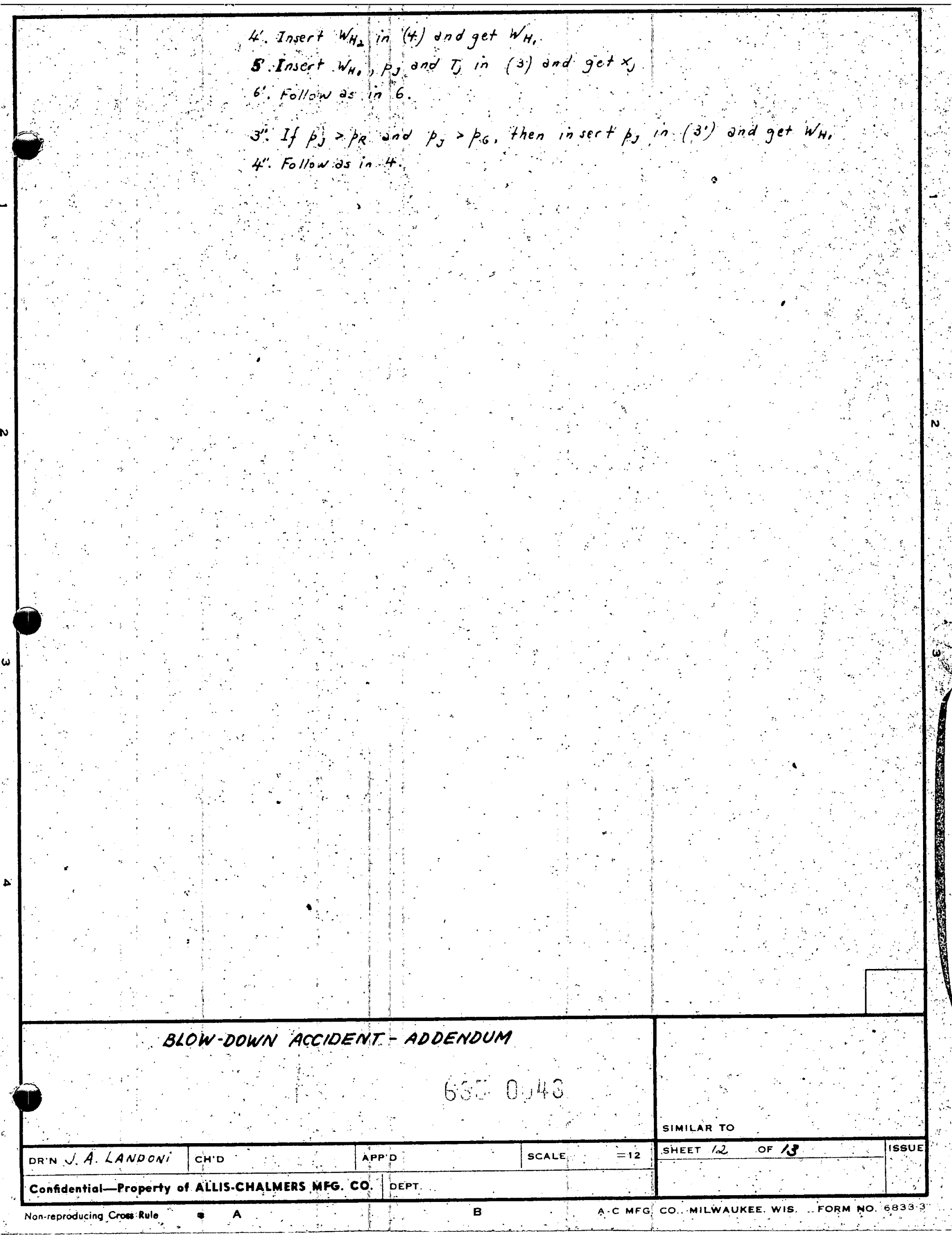


TGGURE 3 TEMAERARE UNAMD MATER LOSS FROM THE PRES SURIZER
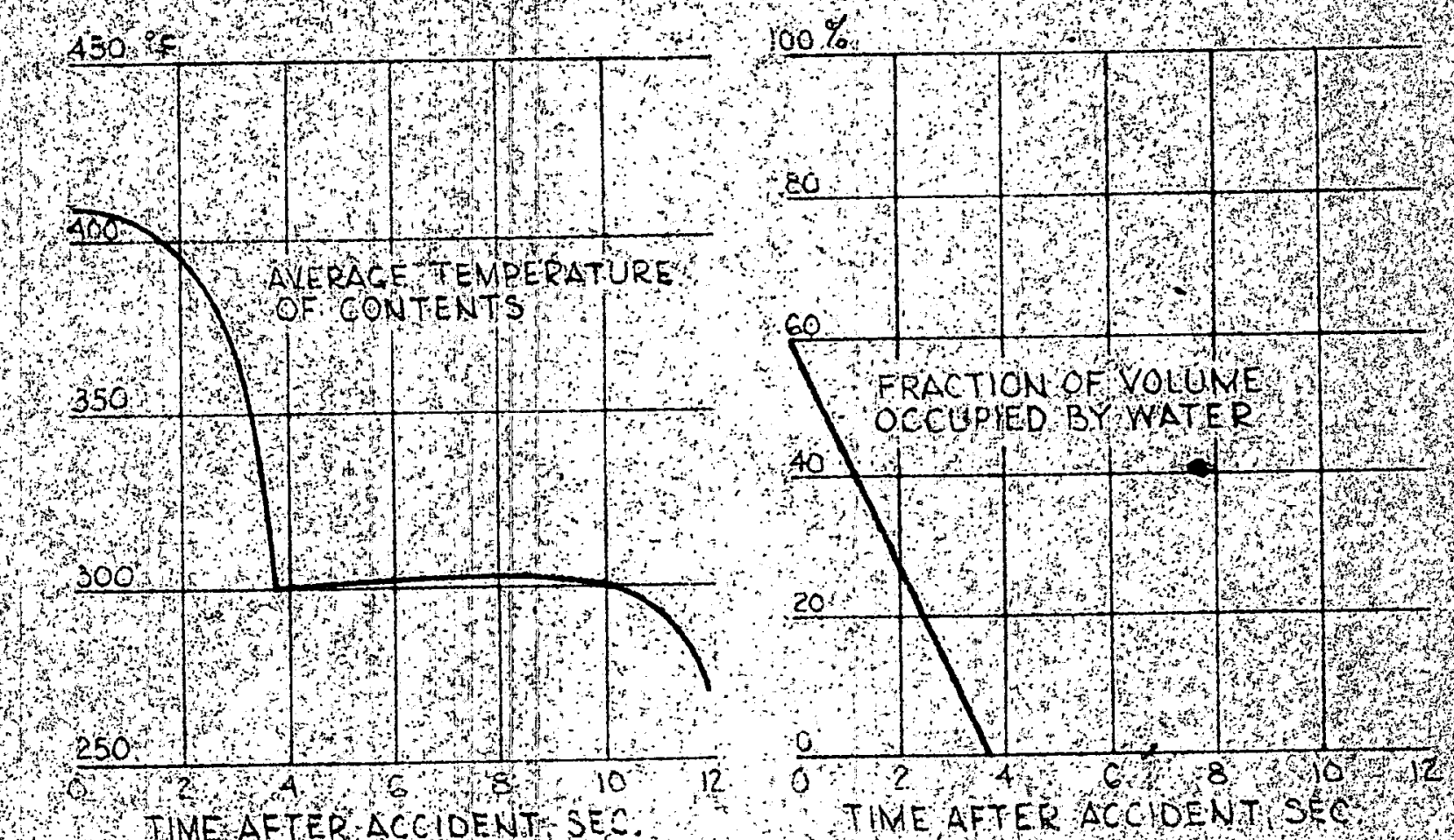

E GURE 4 EXUAUSTMAS FLONRATES

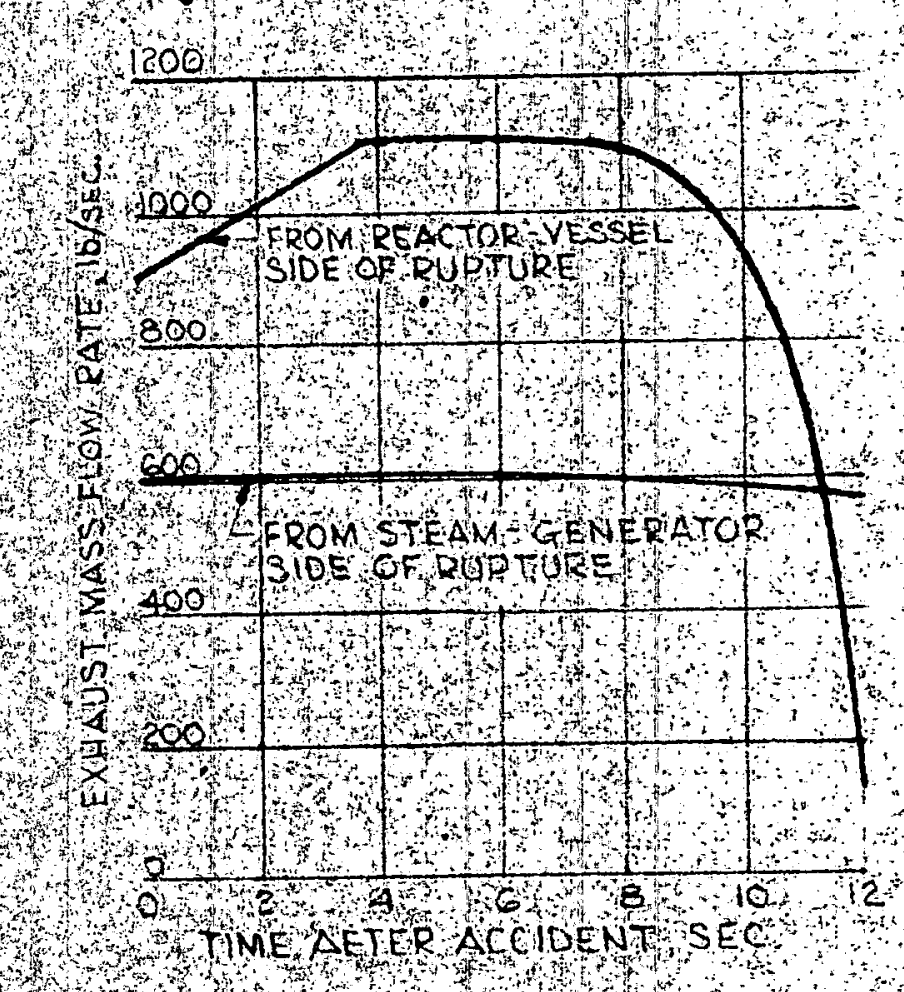




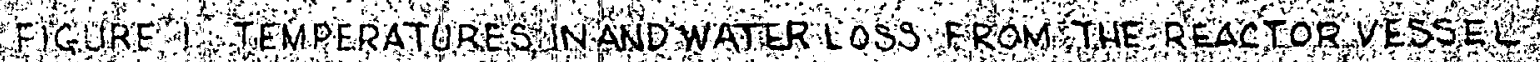
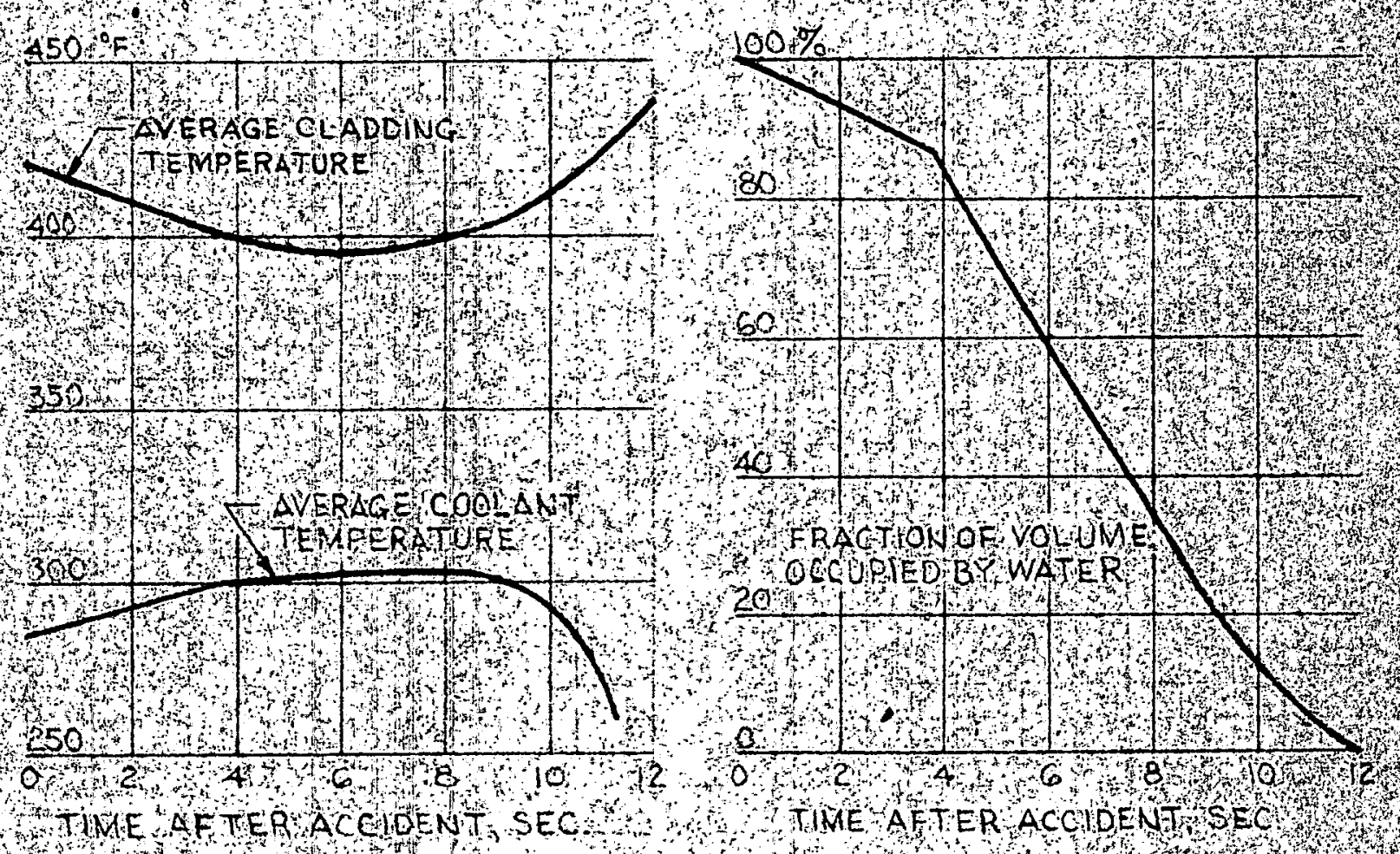

FTCURE 2 TEMPERATURES ANO WATER CONTEN VARIATION WN THESTEAM GENERATOR
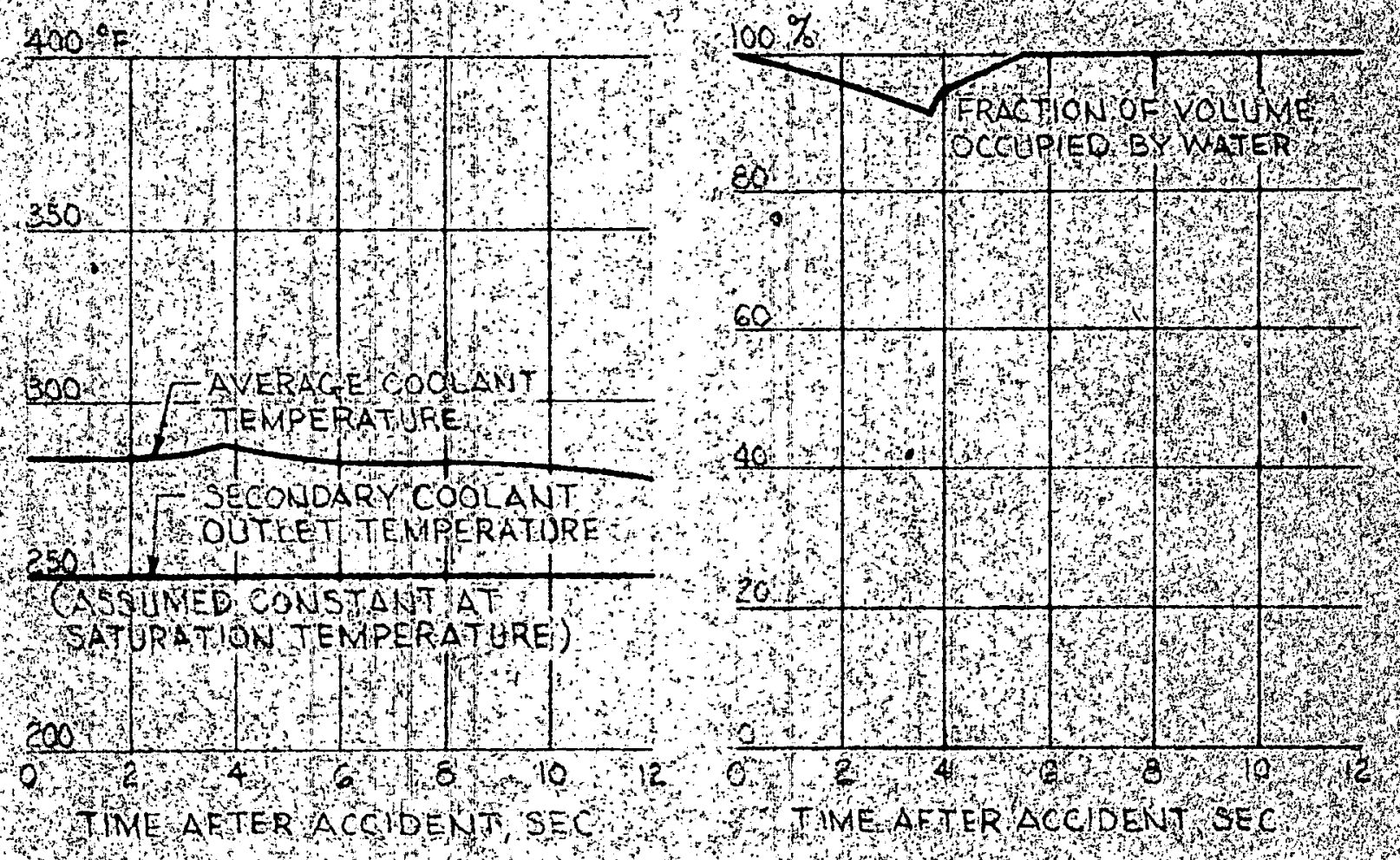

$630 y$ 
APPENDIX II

ANALYSIS OF THE EXTENT OF CLADDING MELTDOWN AFTER THE ELPHR

MAXIMUM CREDIBLE ACCIDENT

\section{CONTENTS}

Est1mate of radial power distribution ............. 1 Constants and initial values ................... 4

Equations for meitdown $\ldots \ldots \ldots \ldots \ldots \ldots \ldots \ldots \ldots \ldots \ldots \ldots \ldots \ldots \ldots$

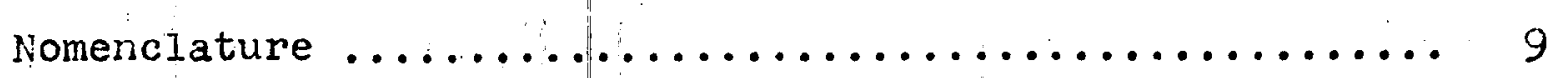

Results ................................ 10

6050.03 


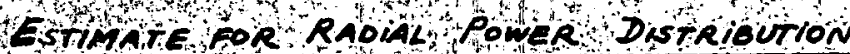

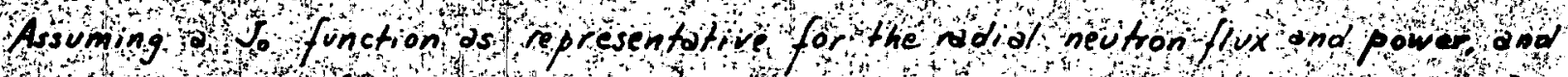

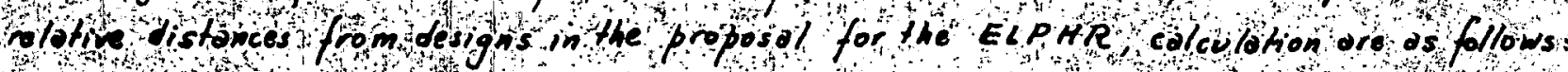

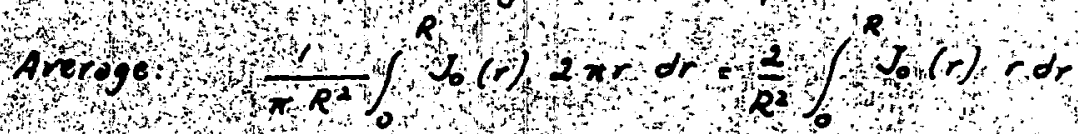

$$
\int(r) r d r(r)
$$

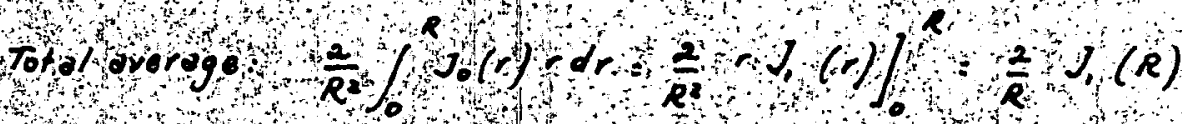

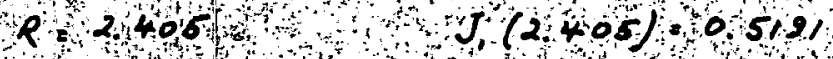

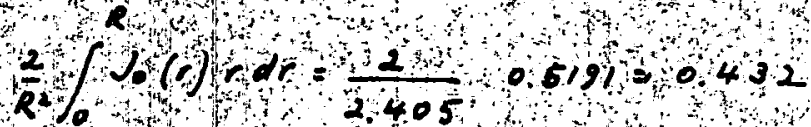

\section{osubojemblies A}

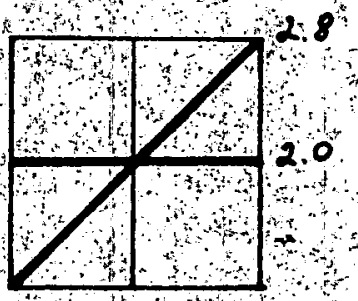

$$
\begin{aligned}
& \text { Aroge } \frac{20+202424}{2}
\end{aligned}
$$

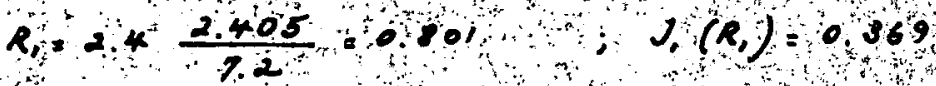

$$
\int_{0}^{2} \int_{0}^{R_{1}}(r) d r=\frac{2}{0.801} 0.3690090
$$

\section{Subossemblies B}

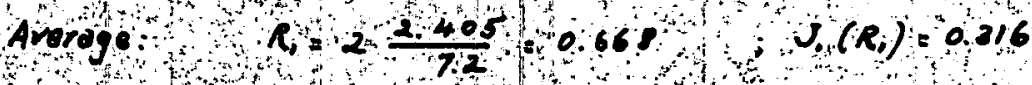

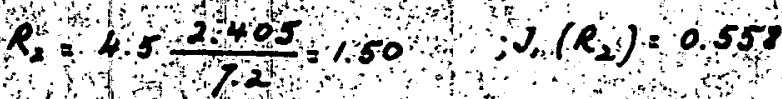

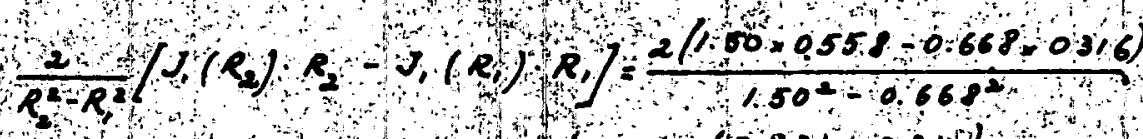

$$
\frac{10.834-0.210)}{(0.232)(2016)}=0 \% 0
$$

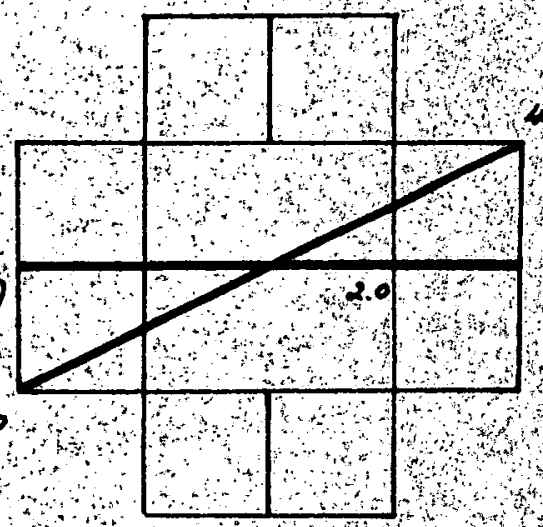

$\omega$

\section{CORE MELT-DOWN}

GIMILAR, TO

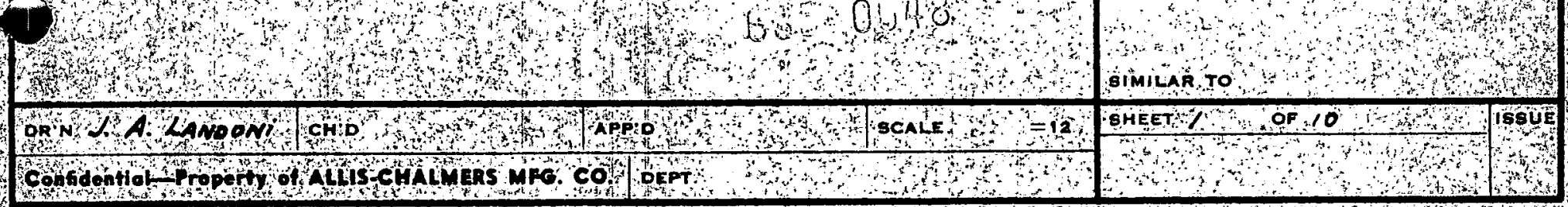




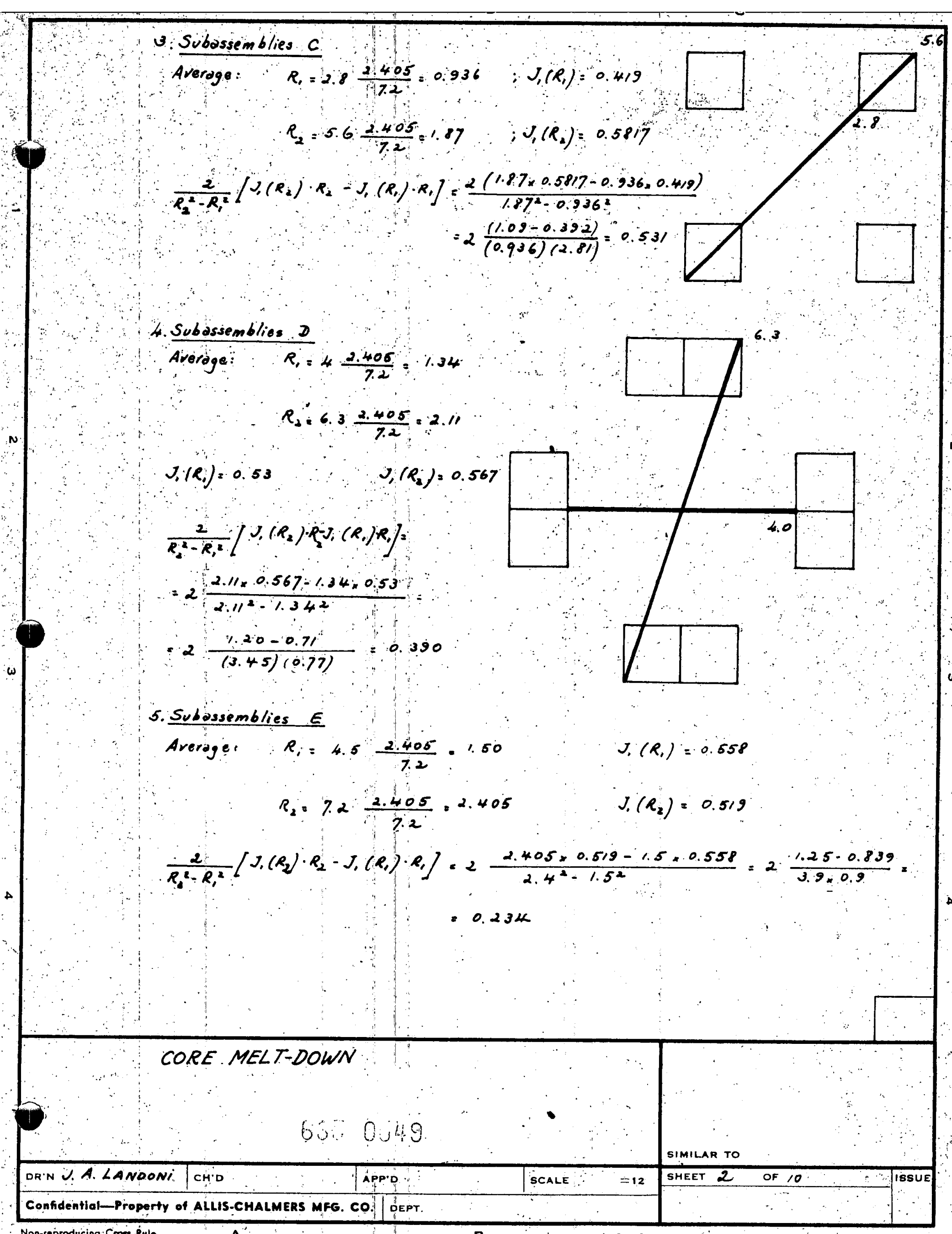

Averoge: $R_{1}=2.8 \frac{2405}{172}=0.936 \quad \because J,\left(R_{1}\right)=0.419$

$R_{2}=5.6 \frac{2.405}{7.2}=1.87 \quad ; V_{1}\left(R_{2}\right)=0.5817$

$\frac{2}{R_{2}^{2}-R_{1}^{2}}\left[J_{1}\left(R_{2}\right) \cdot R_{2}-J_{1}\left(R_{1}\right) \cdot R_{1}\right]=\frac{2(1.87 \times 0.5817-0.936,0.419)}{1.87^{2}-0.936^{2}}$

$=2 \frac{(1.09-0.392)}{(0.936)(2.81)}=0.531$

4. Subossemblies D

Arerage: $\quad R=\frac{2.406}{7.2}=1.34$

$R_{2}=6.3 \frac{2.405}{7.2}=2.11$

$J\left(R_{i}\right)=0.53$

$J_{i}\left(R_{i}\right)=0.567$

$\frac{2}{R_{2}^{2}-R_{1}=}\left[U_{1}\left(R_{2}\right) \cdot R_{2} J_{i}\left(R_{1}\right) R_{1}\right]=$

$=2 \frac{2.11 \times 0.567-1.34 \times 0.53}{2.11^{2}-1.342}=$

$2 \frac{1.20-0.71}{(3.45)(0.77)}=0.390$ 


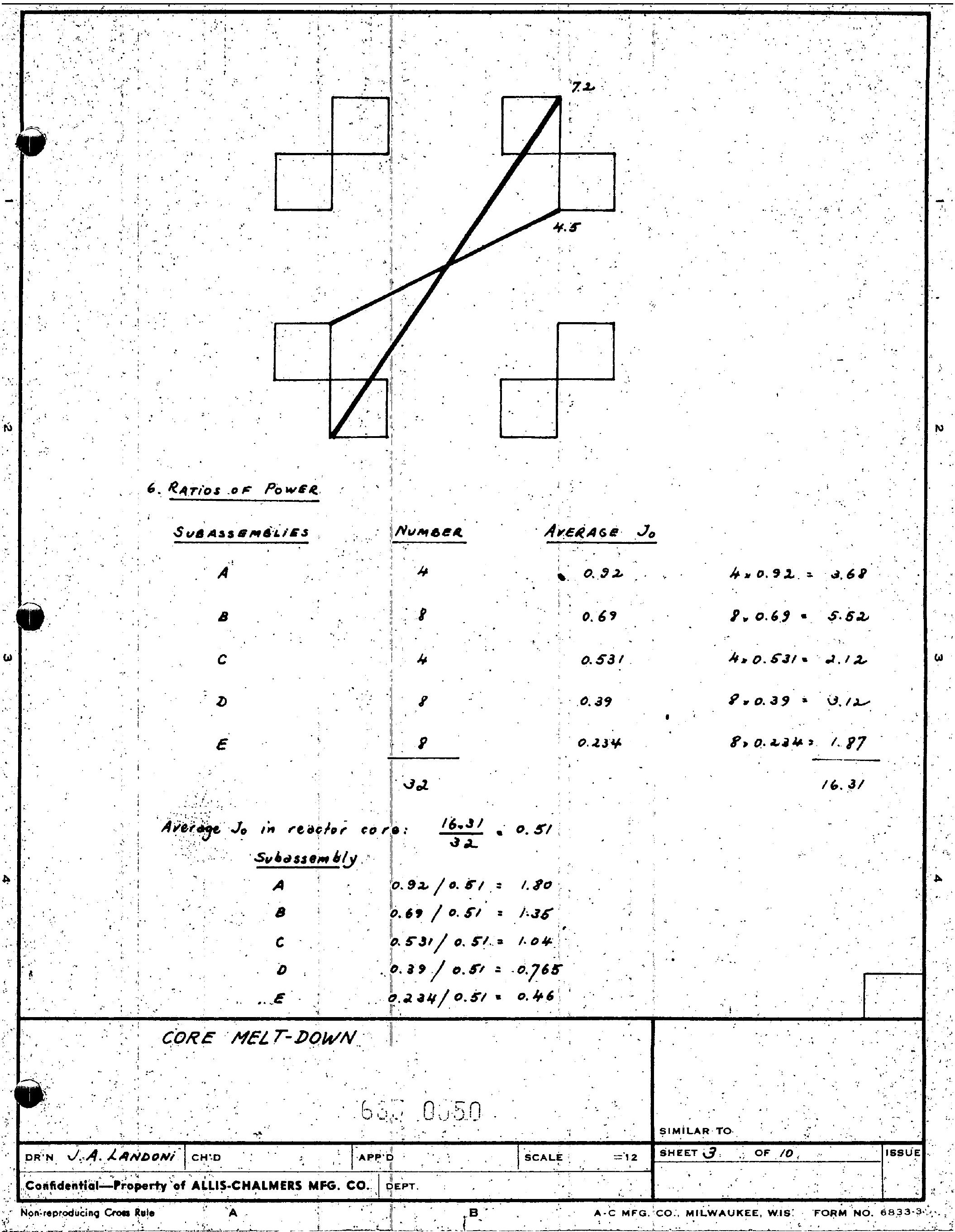




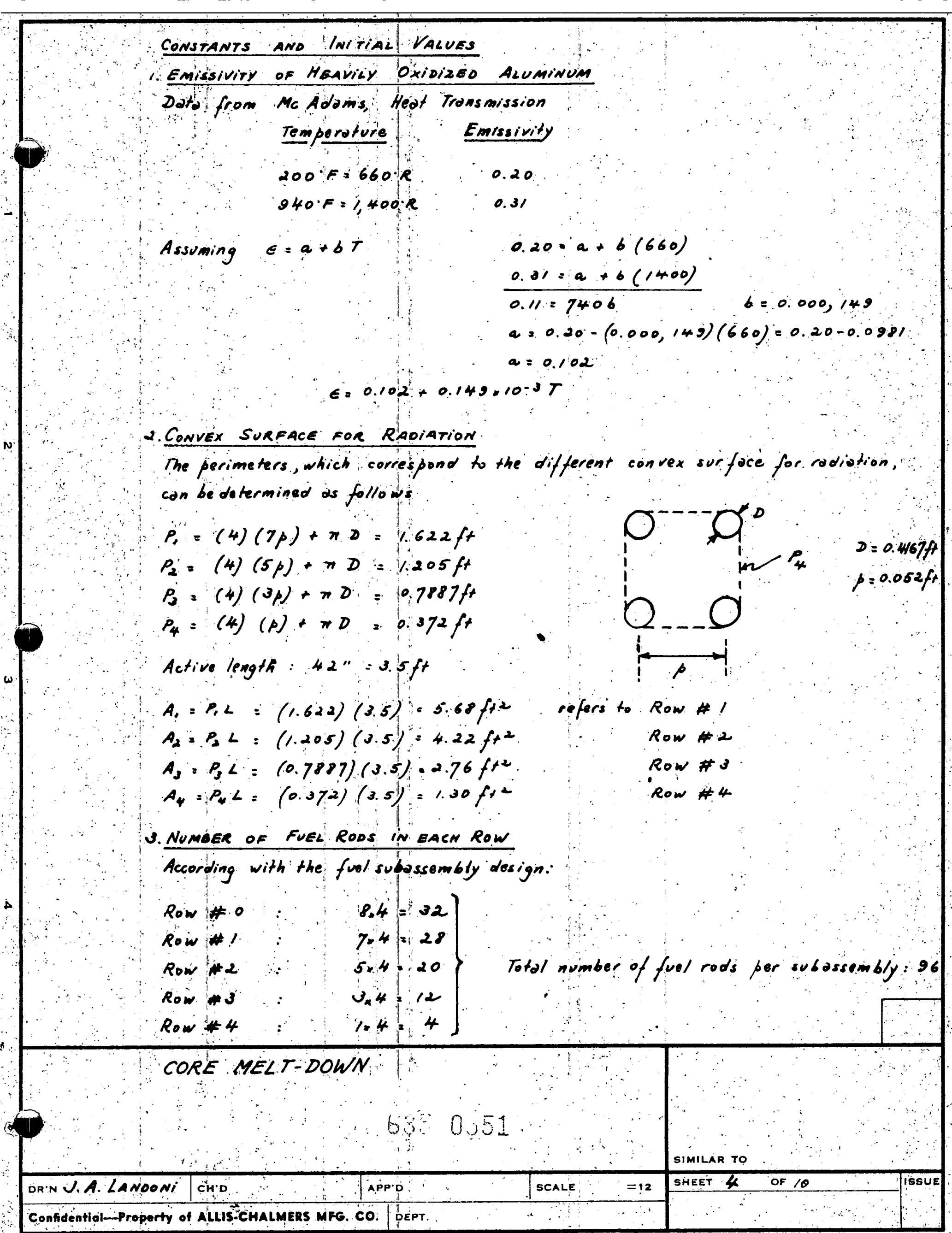


4. Fuel Rods Row Heat CApacitance

Volume of $\mathrm{NO}_{2}$ per rod at room temperature

$$
\dot{\nu}_{v_{2}}=\frac{\pi}{4}(0.417)^{2}(.42)=5.736 \mathrm{in} \mathrm{s} / \mathrm{rod}
$$

Actual density of $\mathrm{vO}_{2}: 10.42 \frac{\mathrm{gm}}{\mathrm{cm}^{2}}=(10.42)(0.0361)=0.376 \frac{\mathrm{ll}}{\mathrm{in}^{3}}$

Weight of $\mathrm{NO}_{2}$ per rod

$$
W_{\mathrm{VO}_{2}}=(5.736)(0.376)=2.16 \mathrm{cb} / \mathrm{rod}
$$

specific heat of $\mathrm{VO}_{2}=0.077 \frac{B T U}{16 \cdot R}$

Volume of Al per rod only in the tubing along the active length at room temperature

$$
V_{A 1}=\frac{\pi}{4}\left(0.5^{2}-0.420^{2}\right)(42)=2.43 \mathrm{in}^{3} / \mathrm{rod}
$$

Density of Al cladding: $2.70 \frac{\mathrm{gm}}{\mathrm{cm}^{2}}=0.0976 \mathrm{cb} / \mathrm{in}^{3}$

Weight of Al tubing per rod

$$
W_{A I}=(0.0976)(2.43)=0.237 \mathrm{l6} / \mathrm{rod}
$$

specific hoot of $A 1=0.24 \frac{B T U}{16 \cdot R}$

Fuel Rod Heat Capacitance

Row \#

0

1

2

4
$(2.16)(0.077)+(0.237)(0.24)=0.223 \frac{370}{\text { prod }}$

Heat Capacitance

$$
\begin{aligned}
& F_{0}=(0.223)(32)=7.14 \frac{B T V}{R} \\
& F_{1}=(0.223)(28)=6.25 \frac{B T U}{R} \\
& F_{2}=(0.223)(20)=4.46 \frac{B T U}{R} \\
& F_{3}=(0.223)(12)=2.68 \frac{B T U}{R} \\
& F_{4}=(0.223)(4)=0.892 \frac{A T U}{R}
\end{aligned}
$$

5. WATER INUECTION CHARACTERISTICS

Total moss flow $W=409 p m=20 \times 10^{3} \mathrm{ll} / \mathrm{hr}$

$\left.\begin{array}{l}\text { Inlet Temperature: } 70 \cdot \mathrm{F} \\ \text { Exit Temperature } 212: F\end{array}\right\} \quad \Delta T_{w}=2 / 2-70=142 \cdot \mathrm{F}$

specific Heat of water $c_{p}=1 \frac{\text { BTu }}{16: F}$

$$
\begin{aligned}
& W c_{p} \Delta T_{\mu}=\left(20.10^{2}\right)(1)(1+2)=2.84 \times 10^{6} \frac{\Delta T U}{h r} \\
& =789 . \frac{8 T 4}{\mathrm{SeC}}
\end{aligned}
$$

CORE MELTDOWN

\begin{tabular}{|l|l|l|l}
\hline OREN J.A. LANDON & CAD \\
\hline
\end{tabular}

SCALE

$=12$

SIMILAR TO

Confidential-Property of ALLIS-CHALMERS MFG. CO: DEPT

Non-reproducing Cross Rule

$\dot{\mathbf{B}}$

A.C MFG. CO.. MILWAUKEE, WIS.

FORM NO. 6833.3

iSSUE

4 
6. AFTERHEAT

6. A. "TEMPERATURE PROFILE in FUEL

Using same dato for Slow down Accident Analysis, p. Is Constants

$$
\begin{aligned}
& \lambda=0.1 \text { sec } \\
& c_{2}=28.8 \cdot 10^{j} \text { sTu } \\
& \bar{C}_{2}=\frac{28.8 .10 \%}{96.32}=9.38 \frac{8 T U}{\text { sec }} \text { (total core) (average per fuel rod) }
\end{aligned}
$$

6. B. Fission Products Decay

Sumo reference:

$$
\begin{aligned}
& c_{1}=2.28=10^{3} \text { Btu sec }-0.8 \text { (total core) } \\
& \frac{c_{0}}{0.8}=2.85 \times 10^{3} \text { Brusec }-0.8 \text { (total core } \\
& \frac{c_{1}}{0.8}=\frac{2.85,10^{3}}{96.32}=0.928 \text { BTu sec -0.8 (overage for fuel rod) }
\end{aligned}
$$

7: End or BLow- Donn Conditions

Time: " $t_{0}=12$ sec.

Average cladding temperature: $T=440 F=000 R$

P: CLAD MELTING Point

Melting Pint of pure Al $659.7{ }^{\circ} \mathrm{C}=333 \mathrm{~K}=1679 \cdot R=1219 \mathrm{~F}$ (p. 305 of the Hand boot of Chemistry and Physics, 3 st Edition).

CORE MELTDOWN

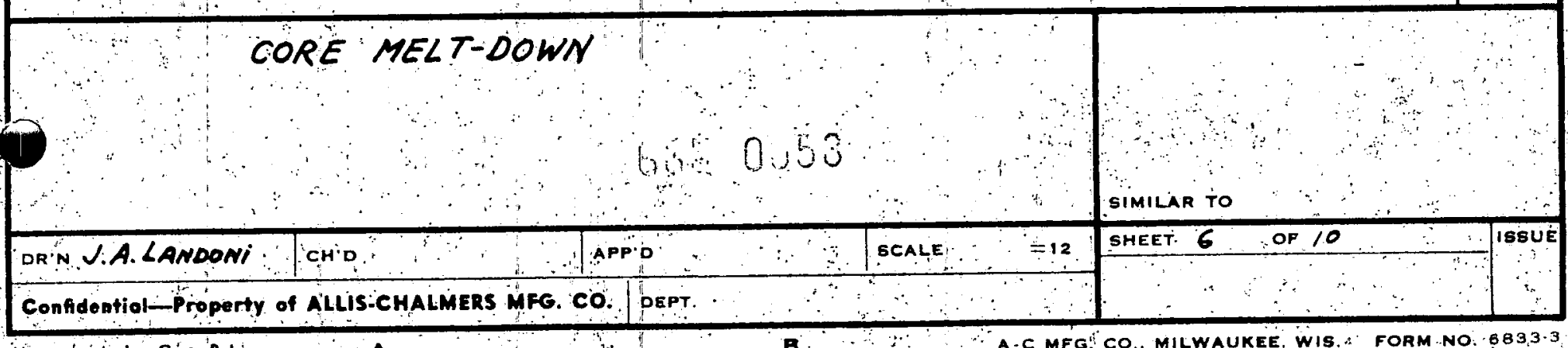

Non-réróducing Cos Rule

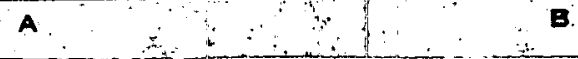

A.C MFGI.CO.. MILWAUKEE, WIS. FORM NO 6833

$N$ 
From pis the fartor for tatio of fluxes (or power) rodially is: 1.80

$$
\begin{aligned}
& \frac{0,0}{0.8}=(0.928)(1.80)(32)=534 \text { Brusec-0.8 } \\
& \because \frac{c_{1}^{\prime}}{0.8}=(0.928)(1.80)(28)=46.8 \text { 8Tu sec-0.8 } \\
& \frac{c_{0}^{2}}{0.8}=(0.928)(1.80)(20)=33.4 \text { BTU sec }^{-0.8} \\
& \frac{c 1^{3}}{0.8}=(0.228)(180)(12)=20.0 \text { oru sec }-0.8 \\
& \frac{c_{0}}{0.8}=(0.928)(1.80)(4)=6.68 \mathrm{Brusec}^{-0.8} \\
& C_{i}^{0}=(9.38)(1.80)(32)=540 \text { Bru sec- }^{-1} \\
& C_{2}^{\prime}=(9.38)(1.80),(28)=473 \text { Bru jec:- } \\
& C_{2}^{2}=(9.38)(1.80)(20)=338 \text { Brusec- } \\
& c_{2}^{3}=(9.38)(1.80)(12)=203 \text { orusec }{ }^{-1} \\
& C_{2}^{*}=(9.08)(1.80)(4)=67.6 \text {. } 870 \mathrm{sec}^{-1} \text {. } \\
& W_{A} c_{p} \Delta T_{w}=(789) \frac{1: 80}{96.32}(96)=44: 4 \frac{\mathrm{Bru}}{\mathrm{sec}}
\end{aligned}
$$

SUBASSEMBLIFS E

From $p$ o the fortor for ralio of flutes (or power) radially is: 0.46 Similarly: as before:

$$
\begin{aligned}
& \frac{c_{1}^{0}}{0.8}=13.7 \text { eru sec-0.8 } \\
& C_{2}^{0}=1388 \mathrm{rUsec}^{-1} \\
& \frac{C_{l}^{\prime}}{0.8}=12.0 \text { BTusec }-0.8 \\
& C_{\alpha^{\prime}}^{\prime}=121 \text { B } \text { TUUec }^{-1} \\
& \frac{c_{1}^{2}}{0.8}=8.53 \text { ervisec-0.8 } \\
& C_{d}^{2}=86.4 \text { Brosec } \\
& \frac{c_{1}^{3}}{0.8}=5.12 \text { arusec }-0.8 \\
& C_{\alpha}{ }^{2}=548 \text { BTU Soc } \\
& \frac{c^{*}}{0.8}=1.7 ! \operatorname{sinsec} 0.8 \\
& C_{2} 4=17.3 \text { ervivect } \\
& W_{E} c_{p} \Delta T_{w}=11.4 \frac{B T U}{S \sigma C}
\end{aligned}
$$

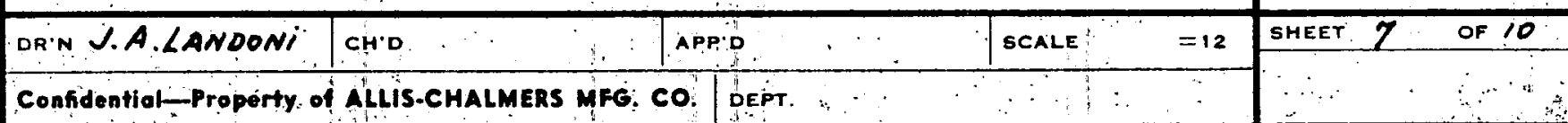


Equations for MELT-DOWN Accident

SUBASSEMBLY $i$

Row \#0 (32. fuel rods)

$$
\begin{aligned}
F_{0} \dot{F}_{0}=c_{1}^{0} t^{-0.2}+C_{2}^{0} e^{-\lambda t} & +\frac{\sigma A_{1}}{\epsilon_{0}^{-1}+\epsilon_{0}^{-1}-1}\left(T_{0}^{+}-T_{0}+\right)-W_{i} c_{p} \Delta T_{w} \\
& \bar{T}_{0} \geq 672 \cdot R
\end{aligned}
$$

Row \#1 (28 fuel rods)

$$
F_{1} \bar{T}_{1}=c_{1}^{\prime} t^{-0 \alpha^{2}}+c_{2}^{\prime} e^{-\lambda t}+\frac{\sigma A_{2}}{\epsilon_{1}^{-1}+\epsilon_{2}^{-1}-1}\left(T_{2}^{*}-T_{1}{ }^{*}\right)-\frac{\sigma A_{1}}{\epsilon_{0}^{-1}+\epsilon_{1}^{-1}-1}\left(T_{1}^{*} T_{0}^{*}\right)
$$

Row * 2 (20 fuel rods)

$$
F_{2} \dot{\bar{T}}_{2}=c_{1}^{2} t^{-0.2}+c_{2}^{2} e^{-\lambda t}+\frac{\sigma A_{0}}{\epsilon_{2}^{-1}+\epsilon_{2}^{-1}-1}\left(T_{2}^{4}-T_{2}^{4}\right)-\frac{\sigma A_{2}}{\epsilon_{1}^{-1}+\epsilon_{2}^{-1}-1}\left(T_{2}^{*} T_{1}^{*}\right)
$$

Row \# 3 (12 fuel rods)

$$
\sigma_{3} \dot{\bar{\sigma}}_{3}=c_{1}^{3} t^{-0.2}+C_{2}^{3} e^{-\lambda t}+\frac{\sigma A_{4}}{\epsilon_{3}^{-1}+\epsilon_{4}^{-1}-1}\left(T_{4}^{4}-T_{3}^{4}\right)-\frac{\sigma A_{3}}{\epsilon_{3}^{-1}+\epsilon_{3}^{-1}-1}\left(T^{4}-T_{2}^{*}\right)
$$

Row \#4 (4 fuel rods)

$$
F_{4} \dot{\bar{T}}_{4}=C_{1}^{*} t^{-0.2}+C_{2}^{4} e^{-\lambda t}-\frac{\sigma A_{4}}{\epsilon_{3}^{-1}+\sigma_{4}^{-1}-1}\left(T_{4}{ }^{4}-T_{3}^{*}\right)
$$

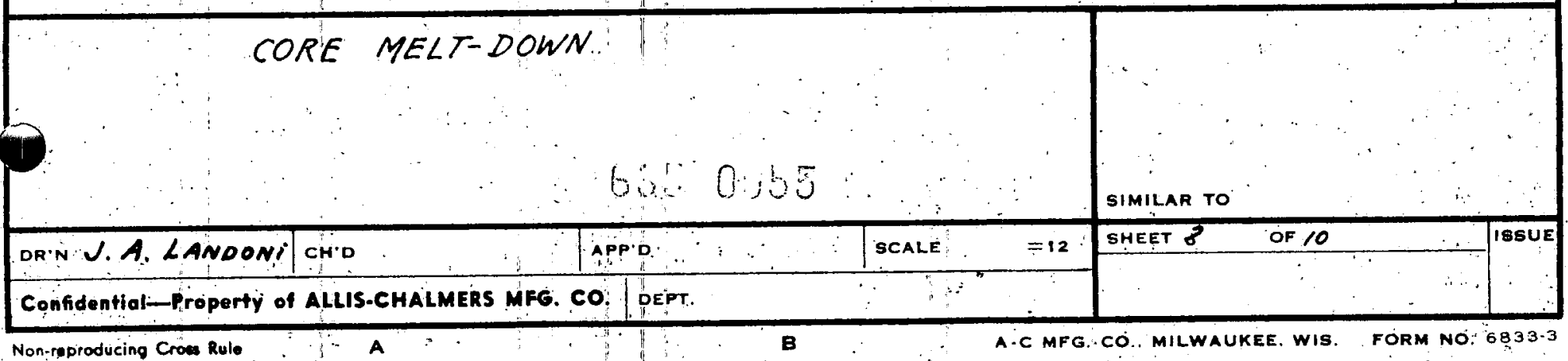

Non-roproducing Crow Rule

A

$\mathbf{B}$

AC MFG CO.. MILWAUKEE. WIS

FORM NO: 6833.3 
NOMENCLATURE

A. area for radiation f te

C. $\because$ constant for fission decay power, Bro sec o.

$C_{2} \quad \therefore$ heat diffusion, BTU/sec:

$\zeta$ specific heat of water $\frac{B T V}{16 \cdot R}$

$F$ fuel rods row he af capacitance, $\frac{\text { TU U }}{V}$

$\overline{\bar{T}}$ absolute temperature $R$

$t$ time after bow-down accident, sec.

$W$ mass flow of water $\frac{16}{\mathrm{sec}}$

$\Delta T_{w}$ temperature rise in the water, $R$

$\in \quad$ emissivity, dimensionless.

$\lambda$ : heat diffusion decay constant, sect

$\sigma$ Stefon-Bo/tzmonn constant, $\frac{B T U / \sec }{f t^{2} \cdot R^{k}}$

subscripts

$\left.\begin{array}{l}0 \\ 1 \\ 3 \\ 4\end{array}\right\}$ refers to different rows in their ares, copacitances, emissivities or temperatures

$i$ different subassemblies in their water moss flow; $A, B, C, D, E$

Superscripts

$\left.\begin{array}{l}0 \\ 2 \\ 4\end{array}\right\}$ refers to different rows in their fission decoy power constants or heat diffusion constants

$\Delta$

$\omega$

CORE MELTDOWN

N. 
Time Requireo to Reach trie Aluminum Melting Point:" $1,219 . \mathrm{F}$ AFrER BLOW-DOWN ACCIOENT : $t_{0}=12 \mathrm{sec}$

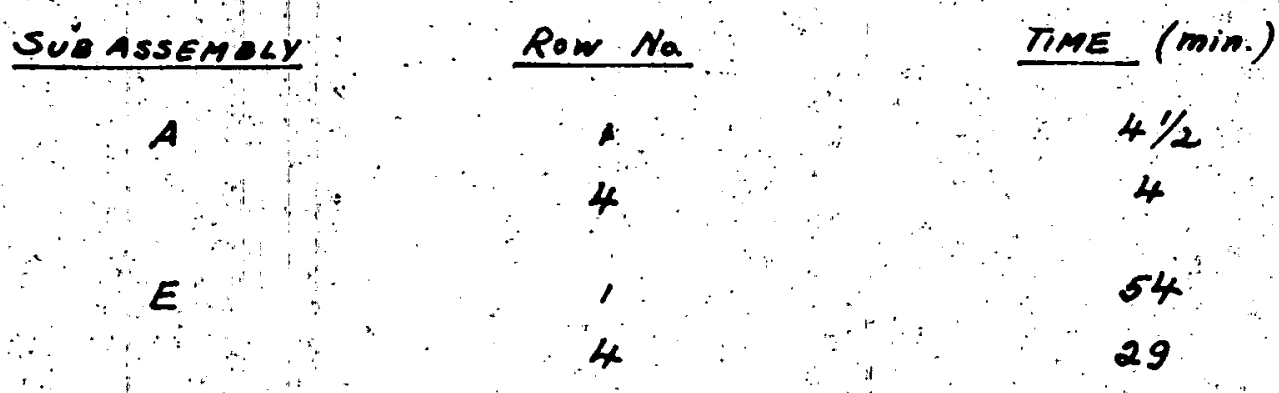

All the fwol rods reach the cladding melting point, except thase locoted on the poriphery of edch subassembly (Row 40 ). Consequently the percent of the core melt-down con be ortimated

$$
\frac{4+i 2+20+28}{4+12+20+28+32}=\frac{64}{96}=2 / 3
$$




\section{APPENDIX III}

ANALYSIS OF THE ENERGY RELEASED FROM THE REACTOR SYSTEM AFTER THE ELPHR MAXIMUM CREDIBLE ACCIDENT

\section{CONTENTS}

Determination of maximum pressures and temperatures in the containment shell ........... I Summary of results...................... 10. Correction for changed primary-system volume....... 11

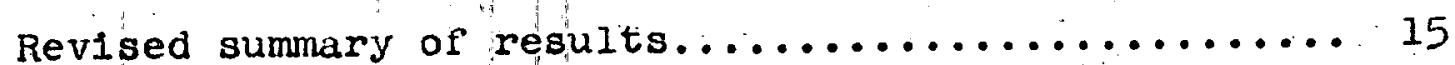


DESIGN CRITERIA FOR CONTAINMENT VESSEL

The maximum pressures and temperatures are produced in fie containment vessel following a rupture of the reactor coolant system.

For this calculation, the chemical reaction of the aluminum, and flashing of the water and steam is assumed to occur instantaneously.

1. Air in the container

Free Volume of Air $r=100,000 \mathrm{ft}$

Ambient Temperature: T=100.F

Pressure: $\quad \beta=14.7$ psia $=1$ atm.

- By the ideal gases law:

$\infty$

$$
\begin{gathered}
p r=n R T=M \frac{R}{\mu} T \\
\mu=28.846 / \mathrm{mole} \\
R=1.545 \frac{16 \mathrm{ft}}{\text { male } R} \\
M=\frac{p V}{\frac{R}{\mu} T}=\frac{(14.7)(144)\left(10^{5}\right)}{\frac{1,545}{28.8}(460+100)}=7.05 \times 10^{\circ 16}
\end{gathered}
$$

2. Water in the reactor system.

Considering a volume of writer which corresponds to 31,00016 for 900 psidinthe system, then at 300 psia, multiplying by the ratio of the specific volumes:

$31,000 \frac{0,0212}{0.0189} * 34,80016$

Water at $285^{\circ} \mathrm{F}: 34,80046$

Water at 4/5 F: $31,000 \mathrm{ll}$

3. steam in the reactor system

In the pressurizer. there ore $23 \mathrm{ft}$. of steam at saturation pressure

At 300 psid

$$
\begin{aligned}
& r=1.543 \mathrm{ft3} / 16 \\
& M_{8}=\frac{23}{1.543}=14.916 \\
& r=0.5006 \mathrm{ft3} / 16 \\
& M_{s}=\frac{23}{0.5006}=46.0 \mathrm{ib}
\end{aligned}
$$

At 900 psid

4. Chemical reaction Al-water

Reaction ${ }_{2} \mathrm{Al}+\mathrm{H}_{2} \mathrm{O} \rightarrow \mathrm{Al}_{2} \mathrm{O}_{3}+\mathrm{H}_{2}$

The standard heat of reaction can be calculated from the heat of formation tables.

CONTAINMENT VESSEL

$$
6300009
$$

SIMILAR TO

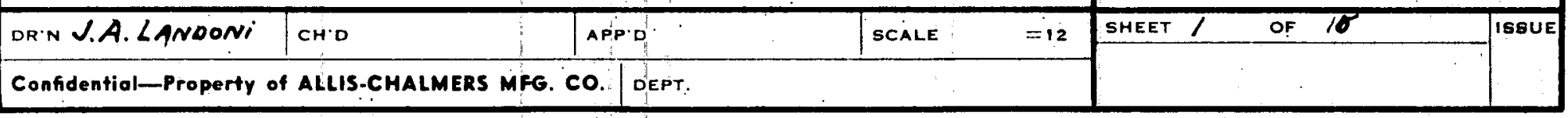

Non-reproducing Crows Rule

A

$\mathbf{B}$

A.C MFG. CO.. MILWAUKEe, WIS.

FOAM NO. 6833.3 


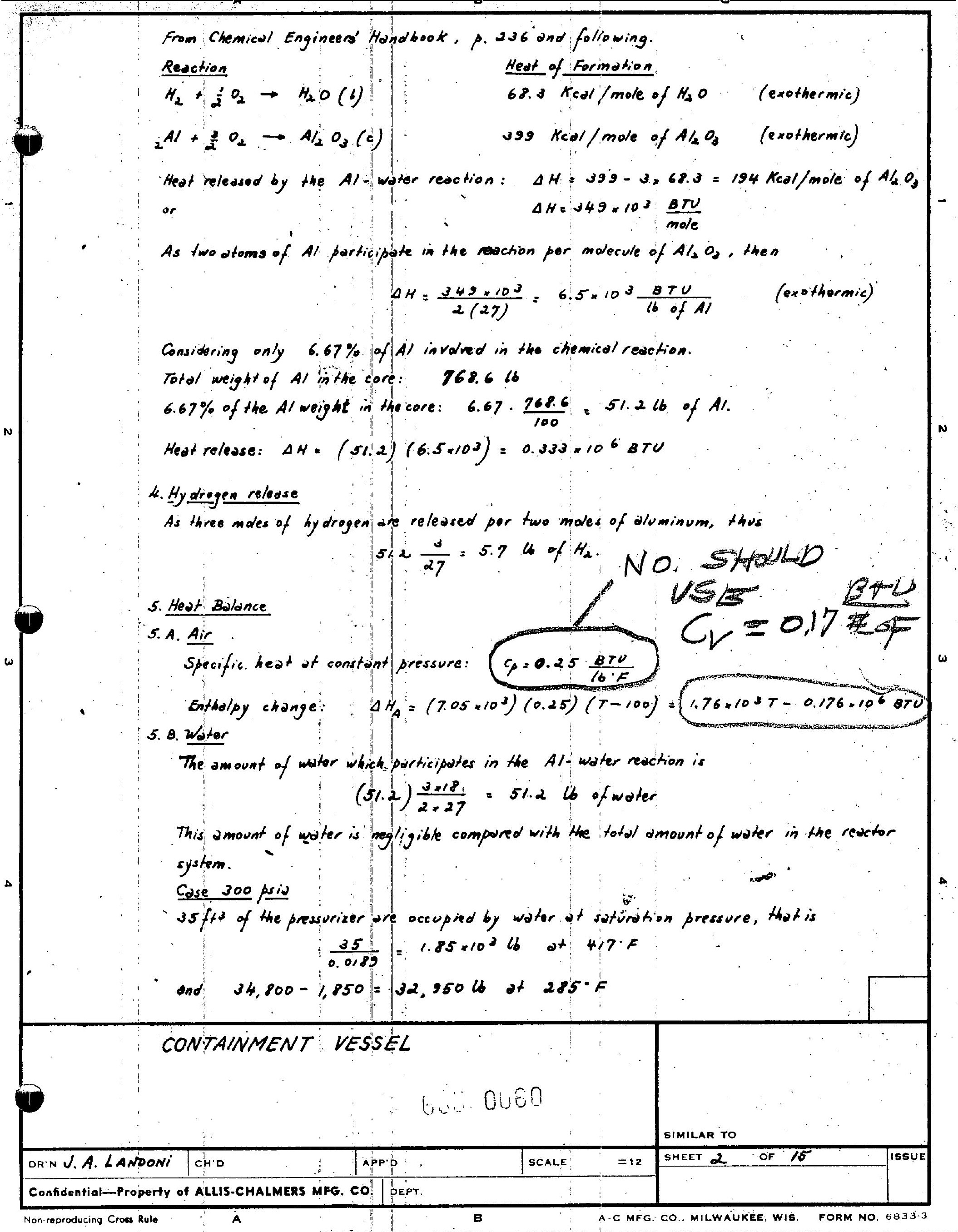




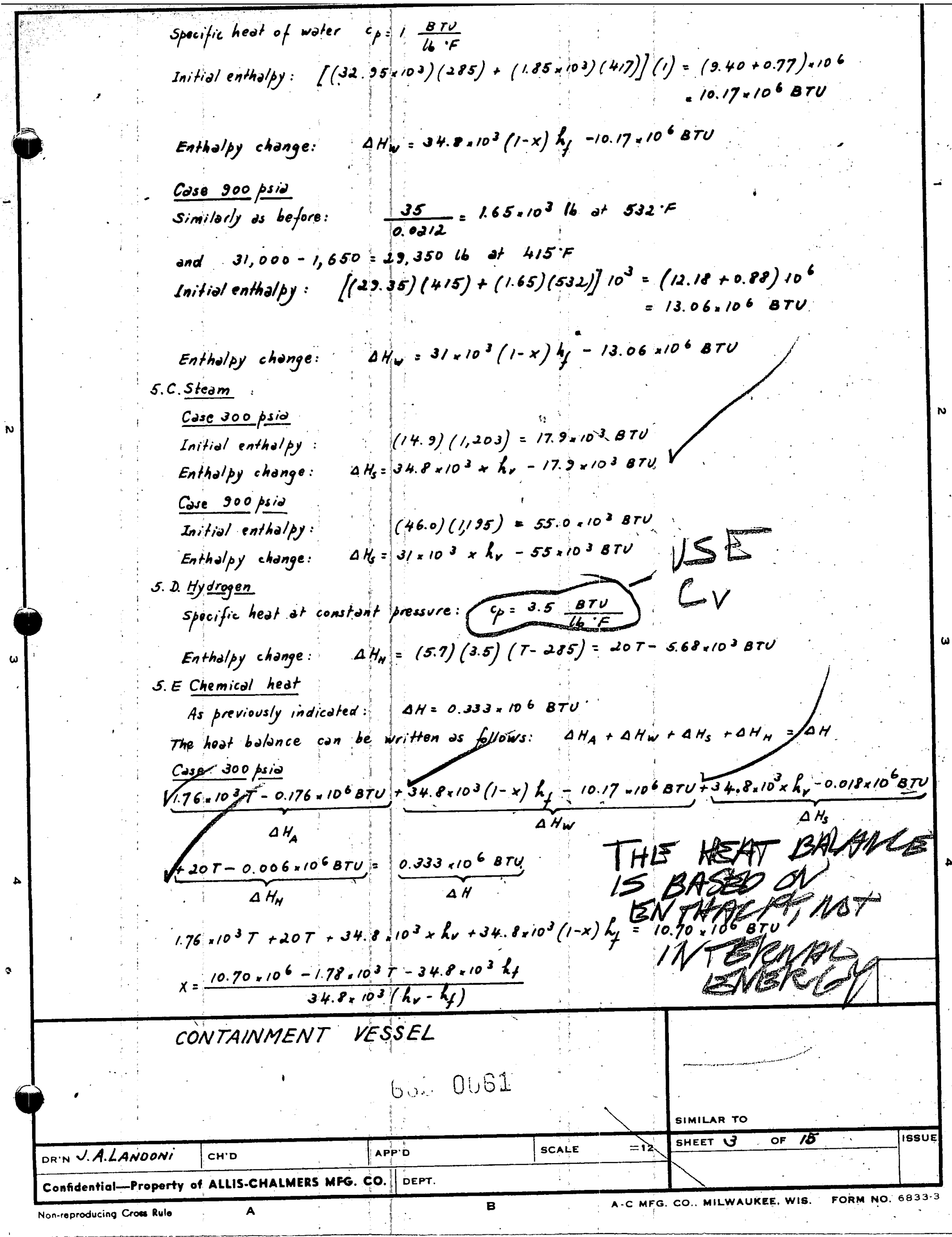




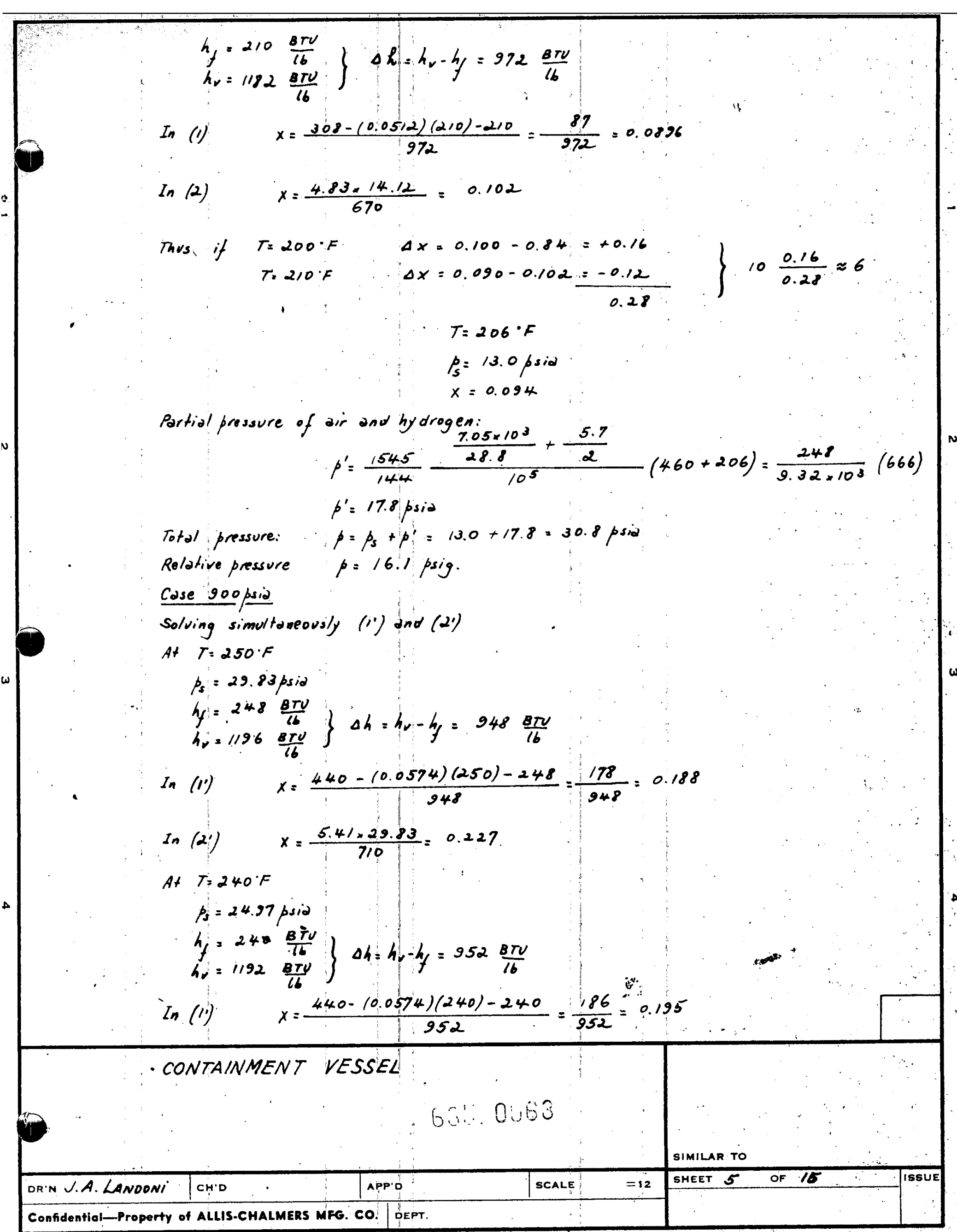

Relative pressure $\beta=16.1$ psig.

Case 900 psia

Solving simultaneously (i) and (2)

At $T=250 . \mathrm{F}$

$p_{s}=29.83 p$ sid

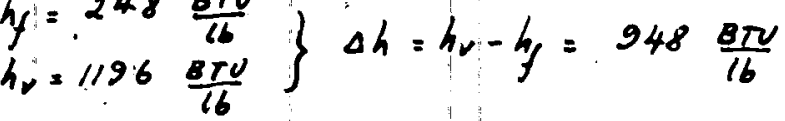

In (19)

$x=\frac{440-(0.0574)(250)-248}{948}=\frac{178}{948}=0.188$

$\ln (2 i)$

$=\frac{5.41 \times 29.83}{710}=0.227$

$T=240 \%$

$p_{s}=24.97$ psid

$h_{f}=24 \cdot \frac{B T}{16}$

$h_{r}=$

$\left.\frac{B T y}{16}\right\}$

$\Delta h=h_{y}-h_{f}=952 \frac{8 \pi v}{16}$

In (19)

$x=\frac{440-(0.0574)(240)-240}{.952}=\frac{186}{952}=0.195$ 


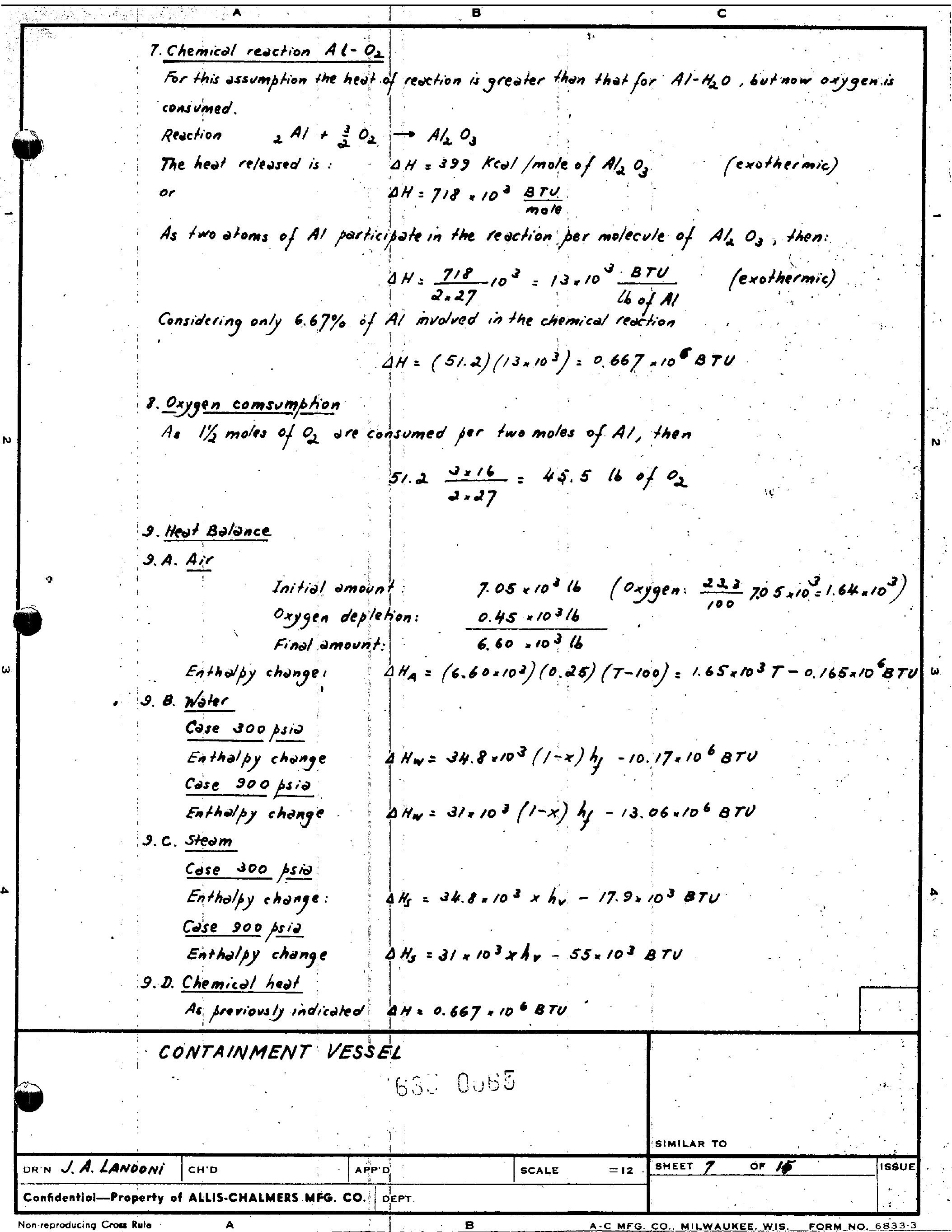




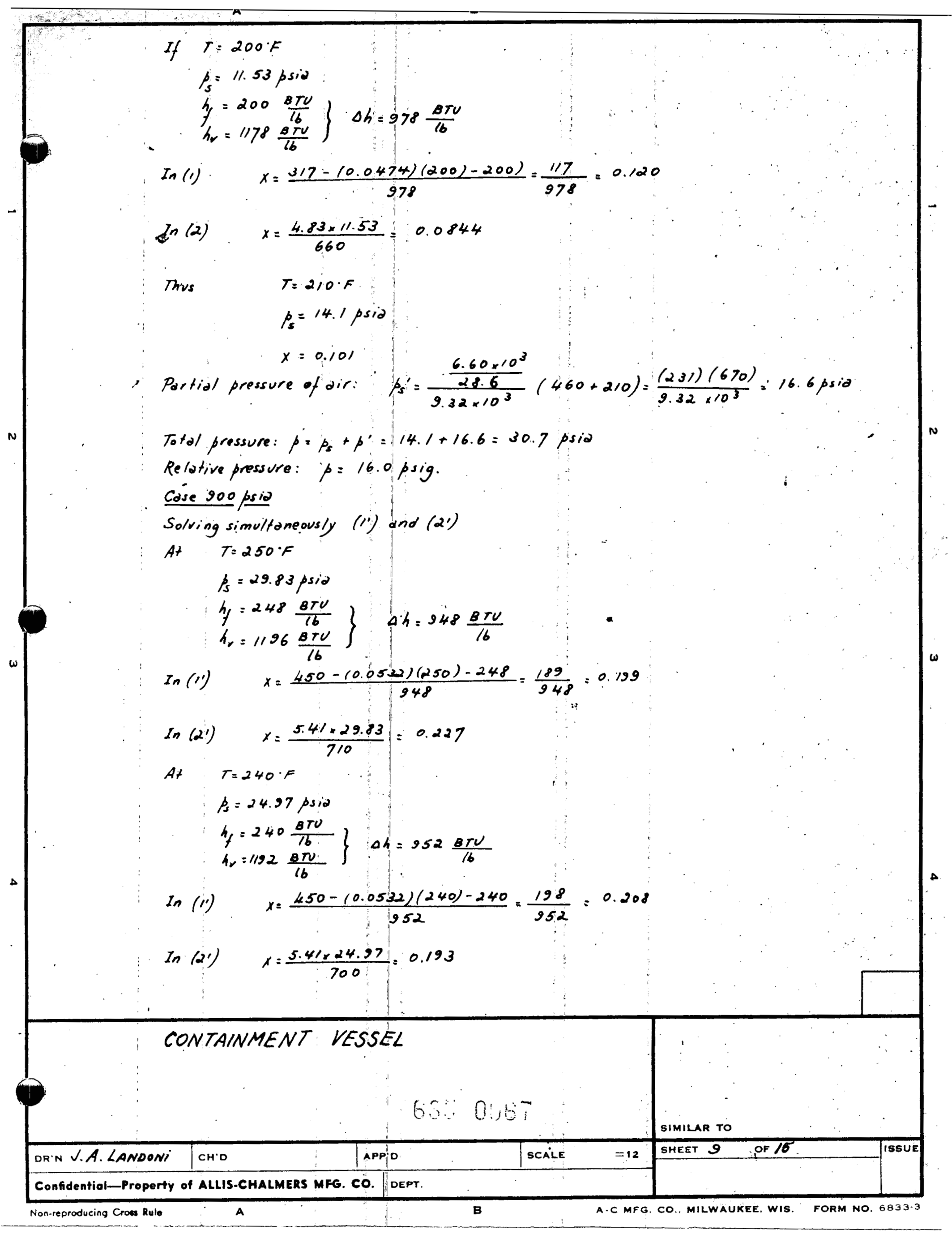




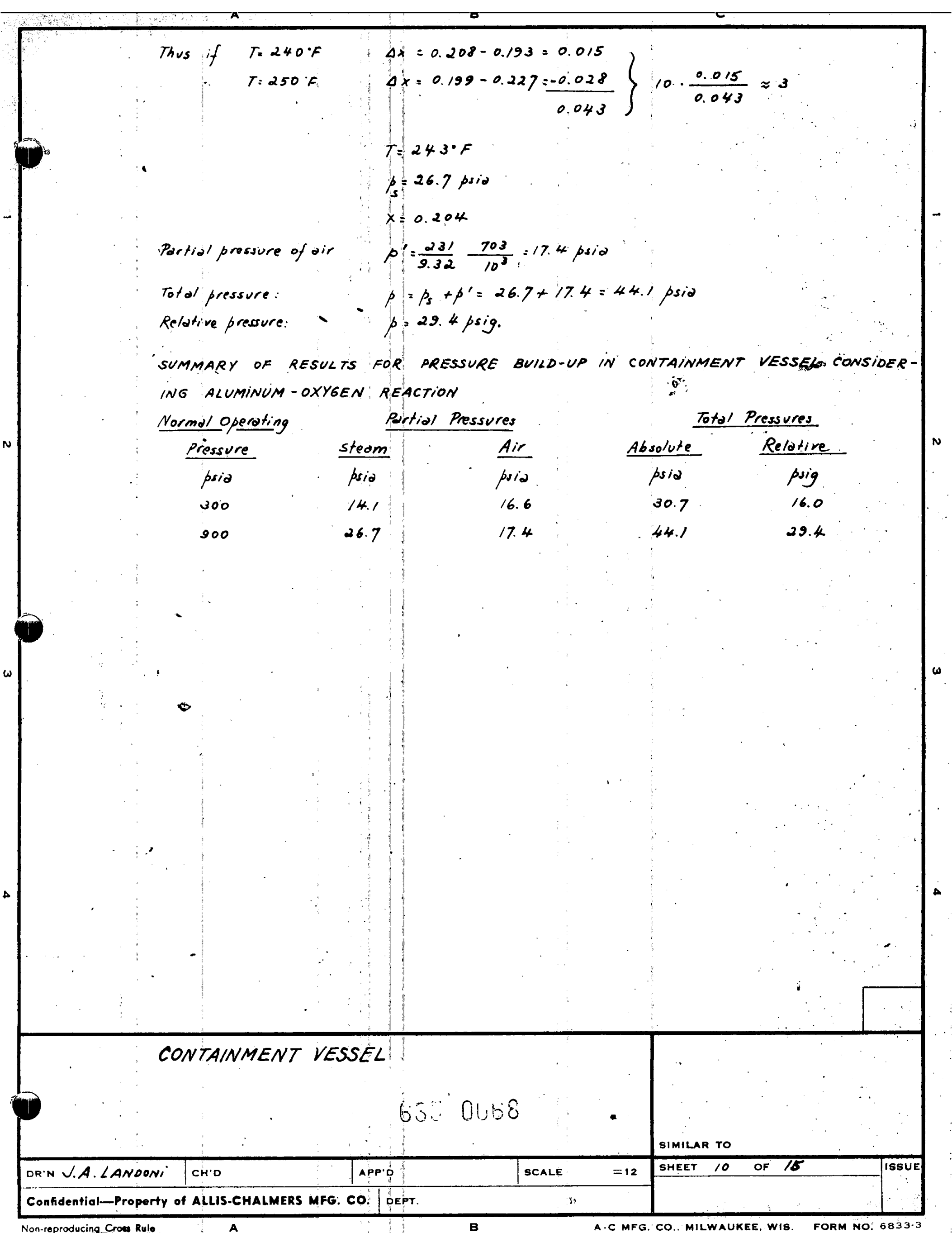




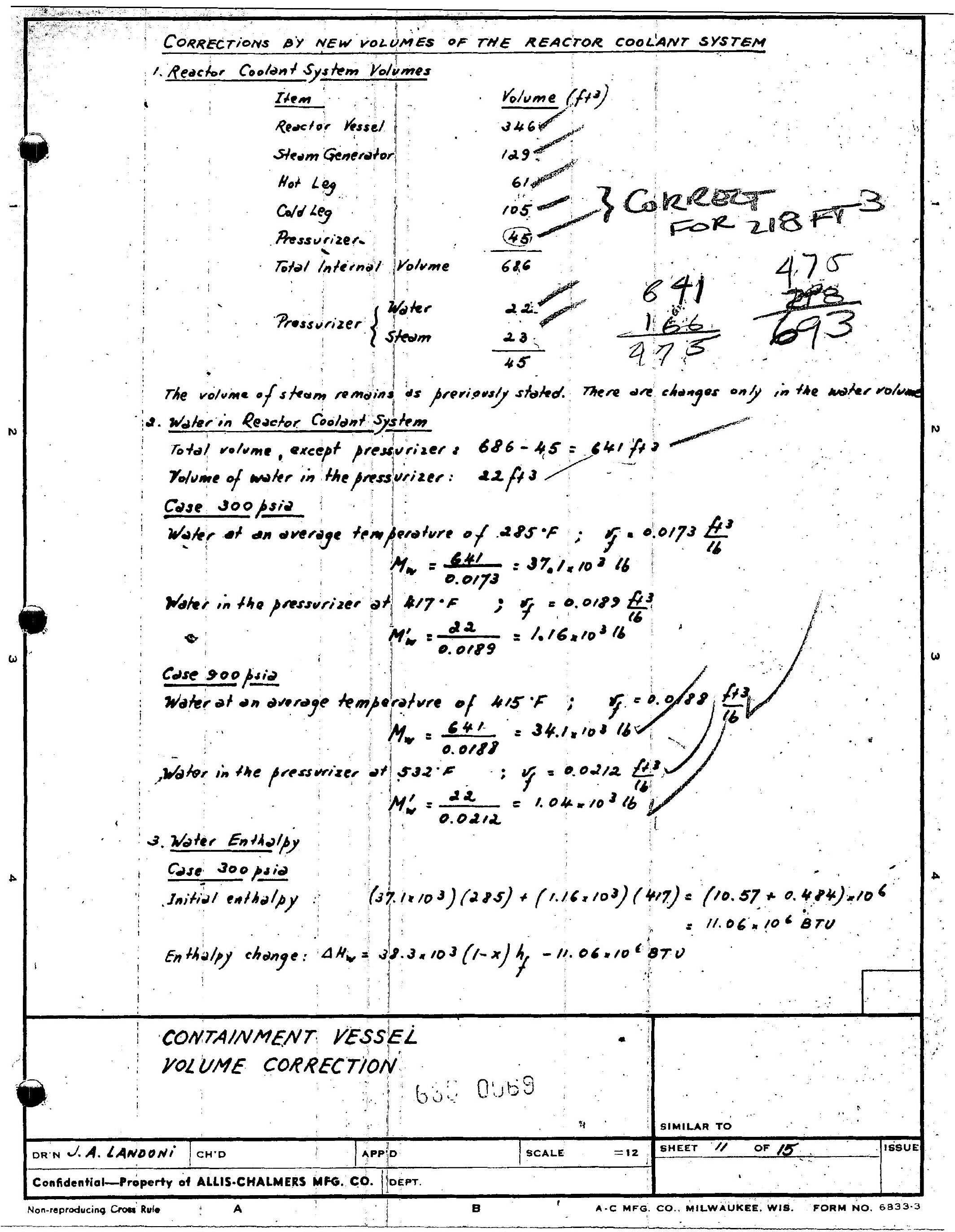




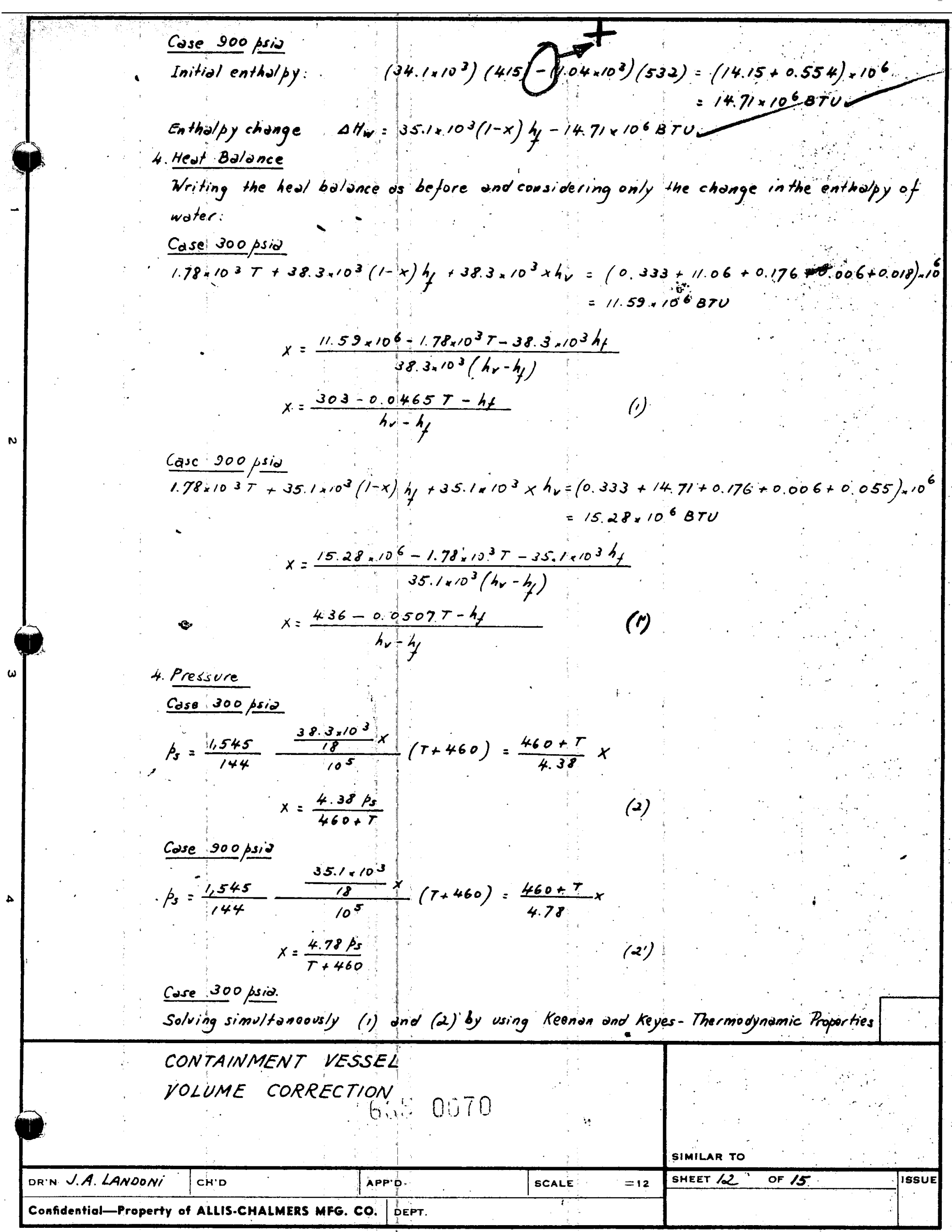

Casc 200 psio

$1.78 \times 10^{3} T+35.1 \times 10^{3}(1-x) h_{1}+35.1 \times 10^{3} \times h_{v}=(0.333+14.71+0.176+0.006+0.055) \times 10^{6}$

$=15.28 \times 10^{6} \mathrm{BTU}$

n

$\omega$ 


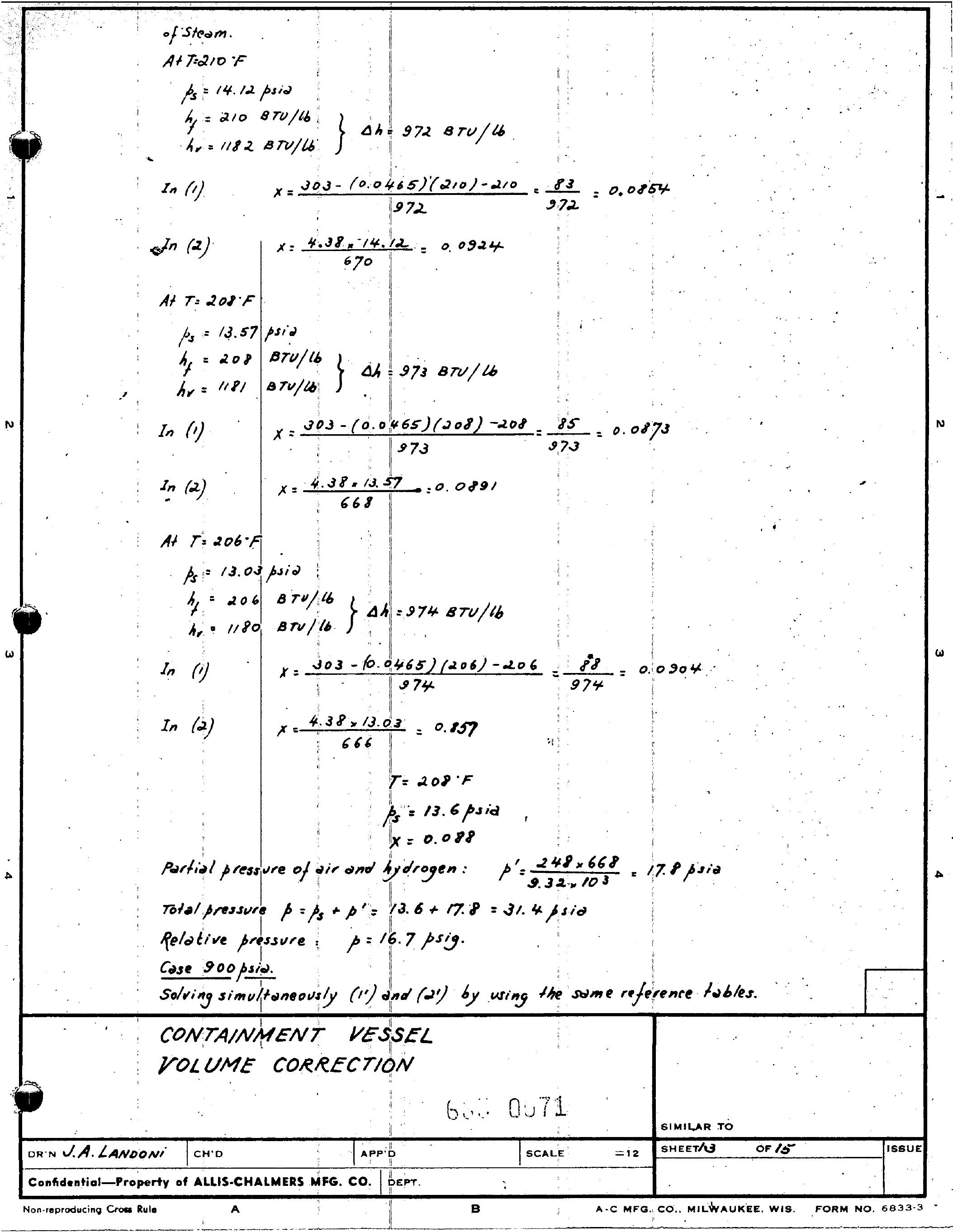




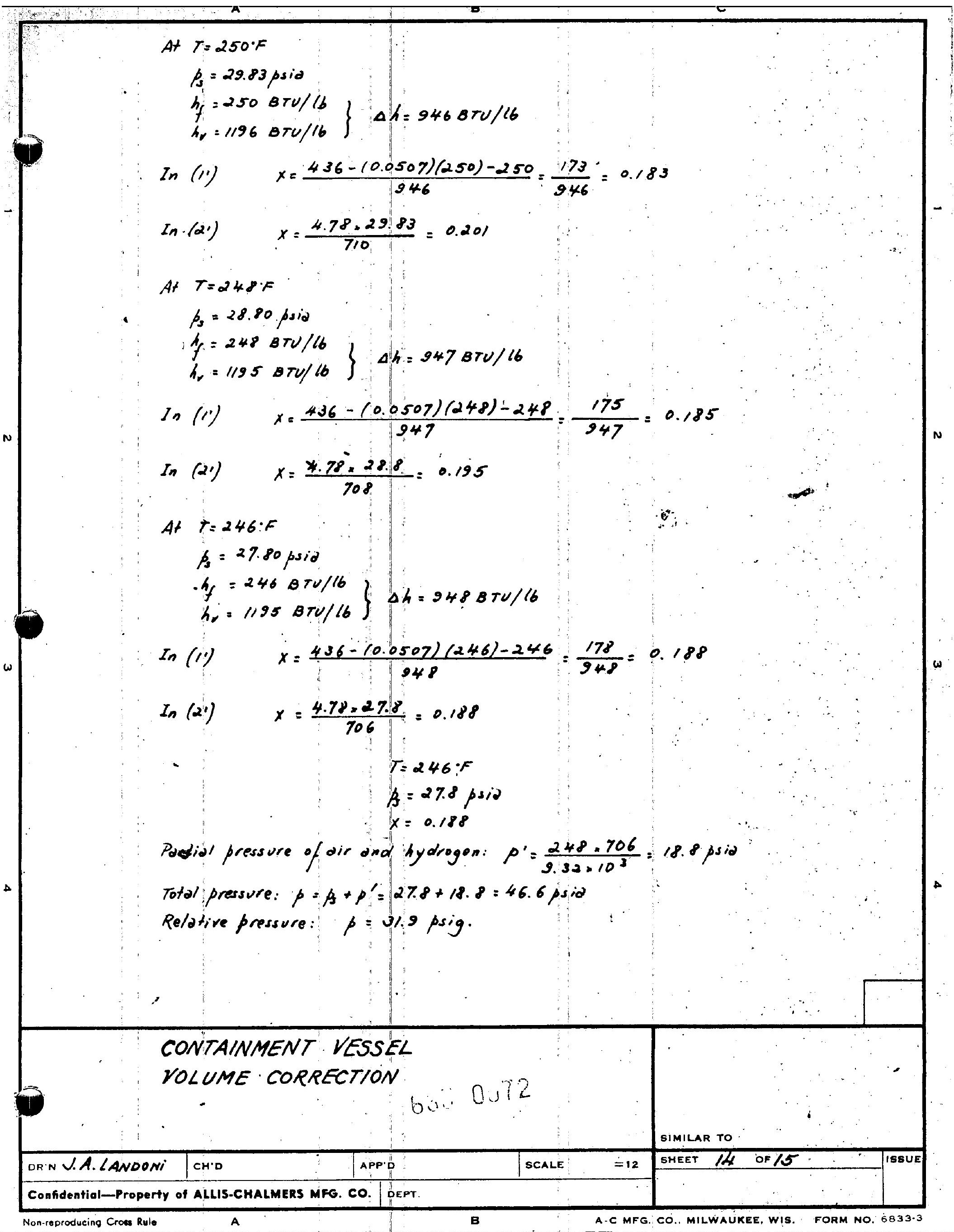




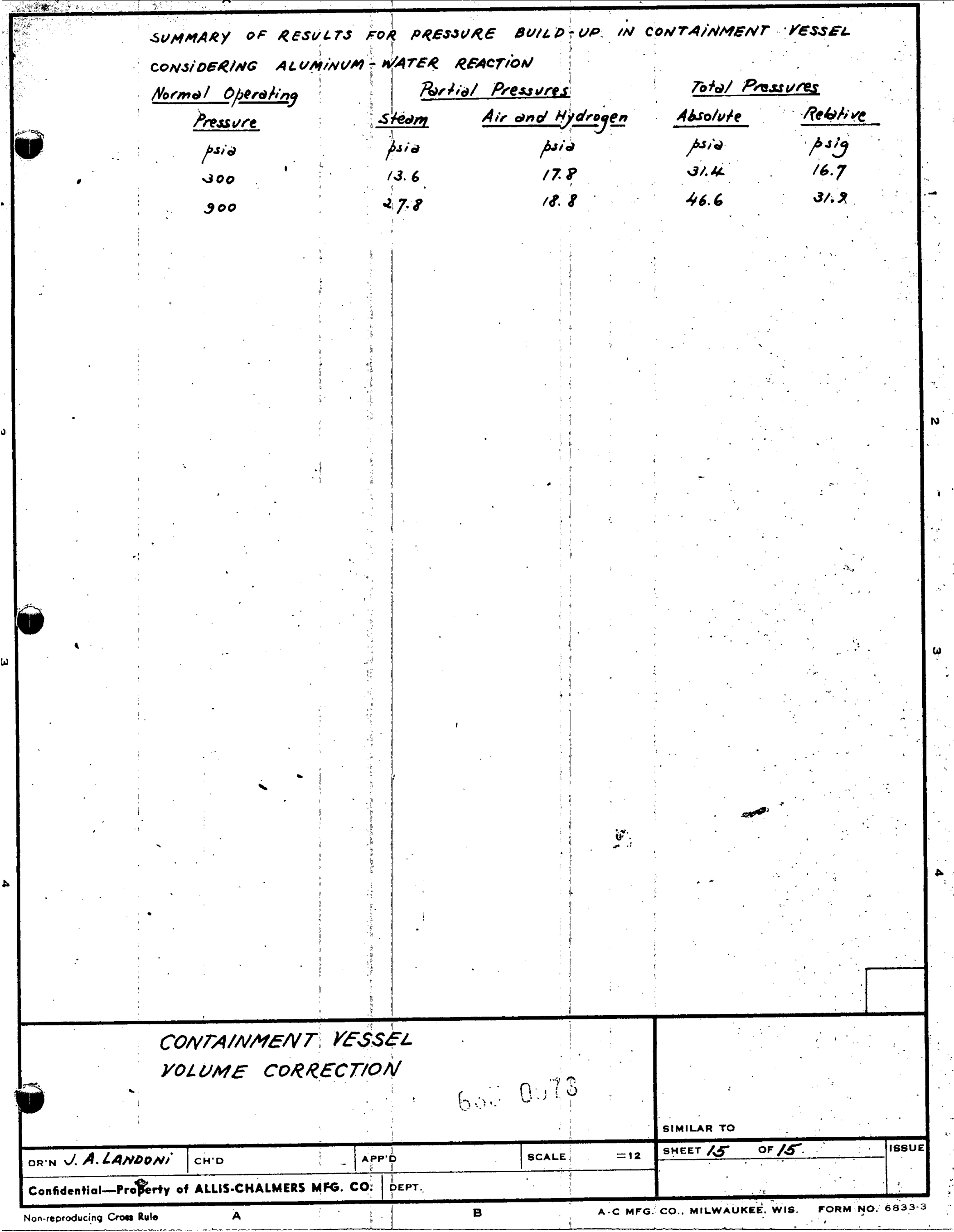




\section{APPENDIX IV}

ANALYSIS OF THE EXTENT OF FISSION-GAS RELEASE AFTER THE ELPHR

\section{MAXIMUM CREDIBLE ACCIDENT}

NoTE: This Appendix contains results applicable to a core with stainless-steel cladding as well as to one with aluminum cladding, because the stainless-steel core was analyzed first and because much of the analysis applies to both cores.

\section{CONTENTS}

Fission-gas release per fuel rod of the stainless steel-clad core ........................... I

Fission-gas reiease from a fuel-element mpture ....... 4 Fission-gas release per fuel rod of the aluminum-

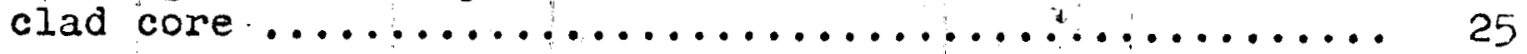

Summary of activity reléase per fuel rod ........... 30

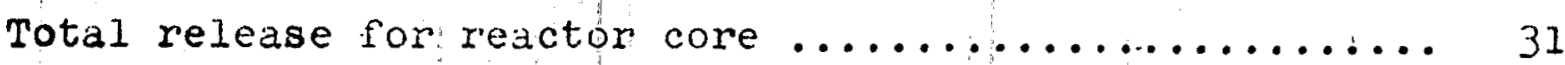
summary of activity release per core .............. 32 


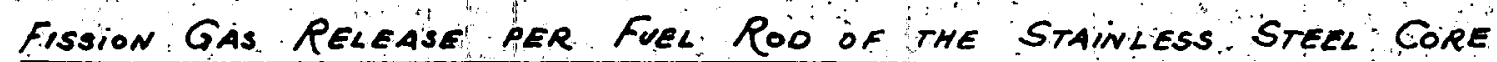
From oRO-196 EGCR Preliminary Hazards Summary Report, p 7.3 , tho activity reledse from the foel is given 6 :

$$
A C T_{i}=\frac{\nu_{i}}{3.70^{\circ}}\left(\frac{y_{i} \sum_{f} \phi \gamma^{10} 0^{16}}{\lambda_{i}+\nu_{i}}\right) \lambda_{i}\left(\frac{1-e^{-\lambda t}}{\lambda}-\frac{e^{-\lambda t}}{\nu}\left(1-e^{-\nu t}\right)\right)
$$

where

$$
\text { ACT: activity of isotope }: \frac{\text { Curie }}{M W}
$$

$\nu_{i} \quad$ experimentally determined escape coefficient for isotope $i$ sec-i

$y_{i} \quad$ fission yield of isotope id dimerisionless

$\Sigma_{1} \quad$ macroscopic fission cress-section; $\mathrm{cm}^{-\prime}$."

$$
\phi \quad \text { inoutron flux } \frac{\text { neutrons }}{\mathrm{cm}^{2} \cdot \mathrm{sec}}
$$

$\lambda_{i} \quad \because$ radiodetive decay constant for isotope is sec-"

$t \quad:$ irradidion time sec

For those is otopes for which soturation is reached the equations become:

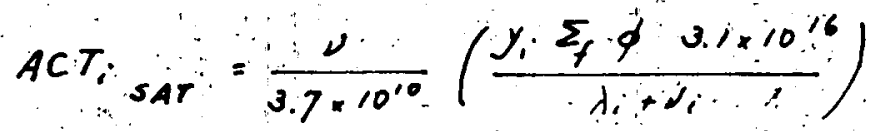

ELPHR Characiteristics (from * Propasal for an Experimental Low Temperature Process Heat Reactor")

Core Life: 3.1 yedrs $=(3.1)(365)(24)(3,600)=0.978-10$ sec.

Reactor Aower: 40.1: MW (thermal)

No: of subassemblies: - 32

No. of rods per subossembly: 100

Power per rod: $\frac{40.1}{32+100}=12.5 \frac{\mathrm{KW}}{\mathrm{rod}}$

References: $A N L=4807$

WAPD -173

WAPO - IS3

ORNL-2505

$0 R 0-196$

\begin{tabular}{|c|c|c|}
\hline OR'N LANOONI: $\mathcal{A} A$. & CH'D & APP'D \\
\hline
\end{tabular}

FISSION GAS RELEASE

6000000

SIMILAR TO

Confidential-Property of ALLIS-CHALMERS MFG: CO 


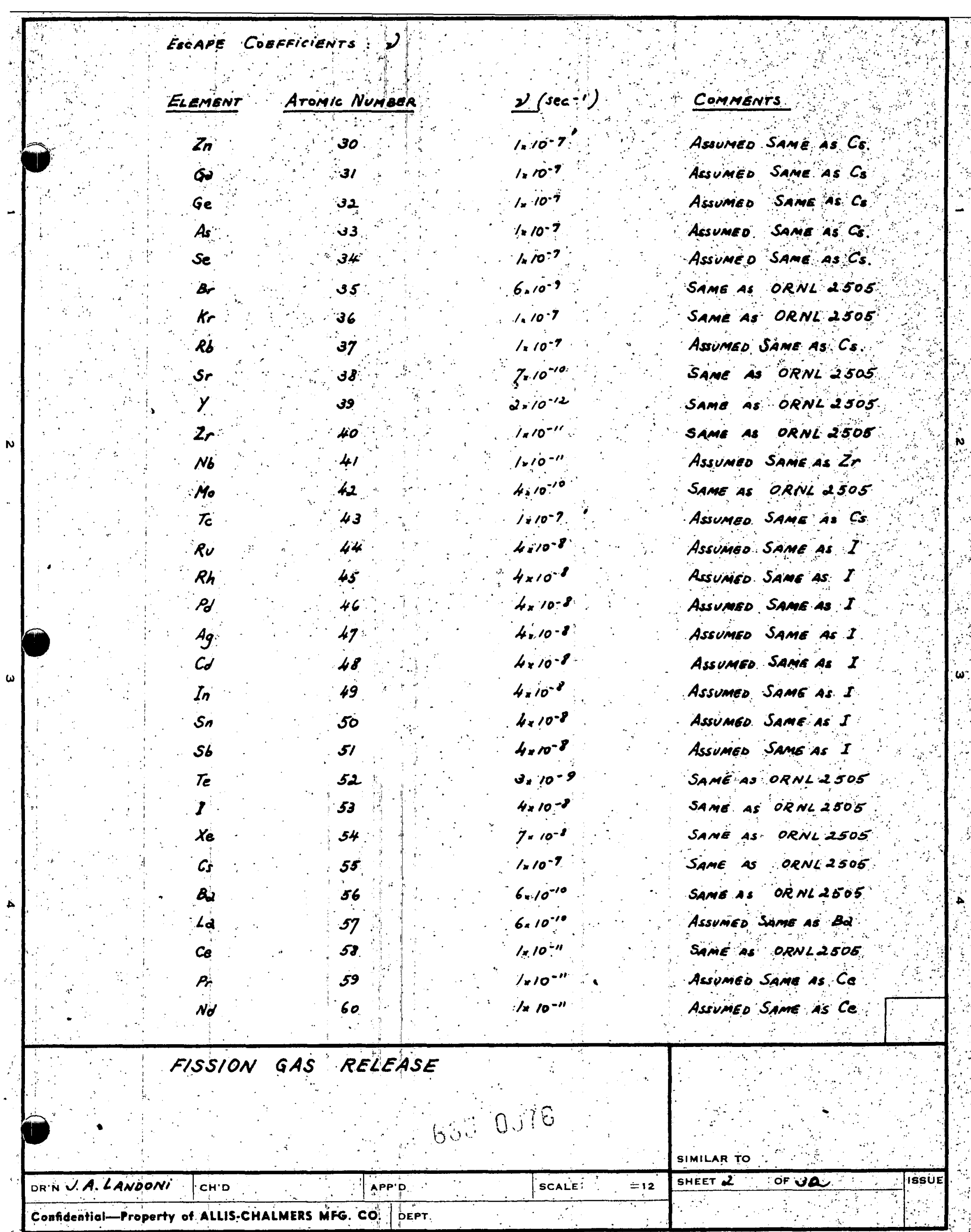




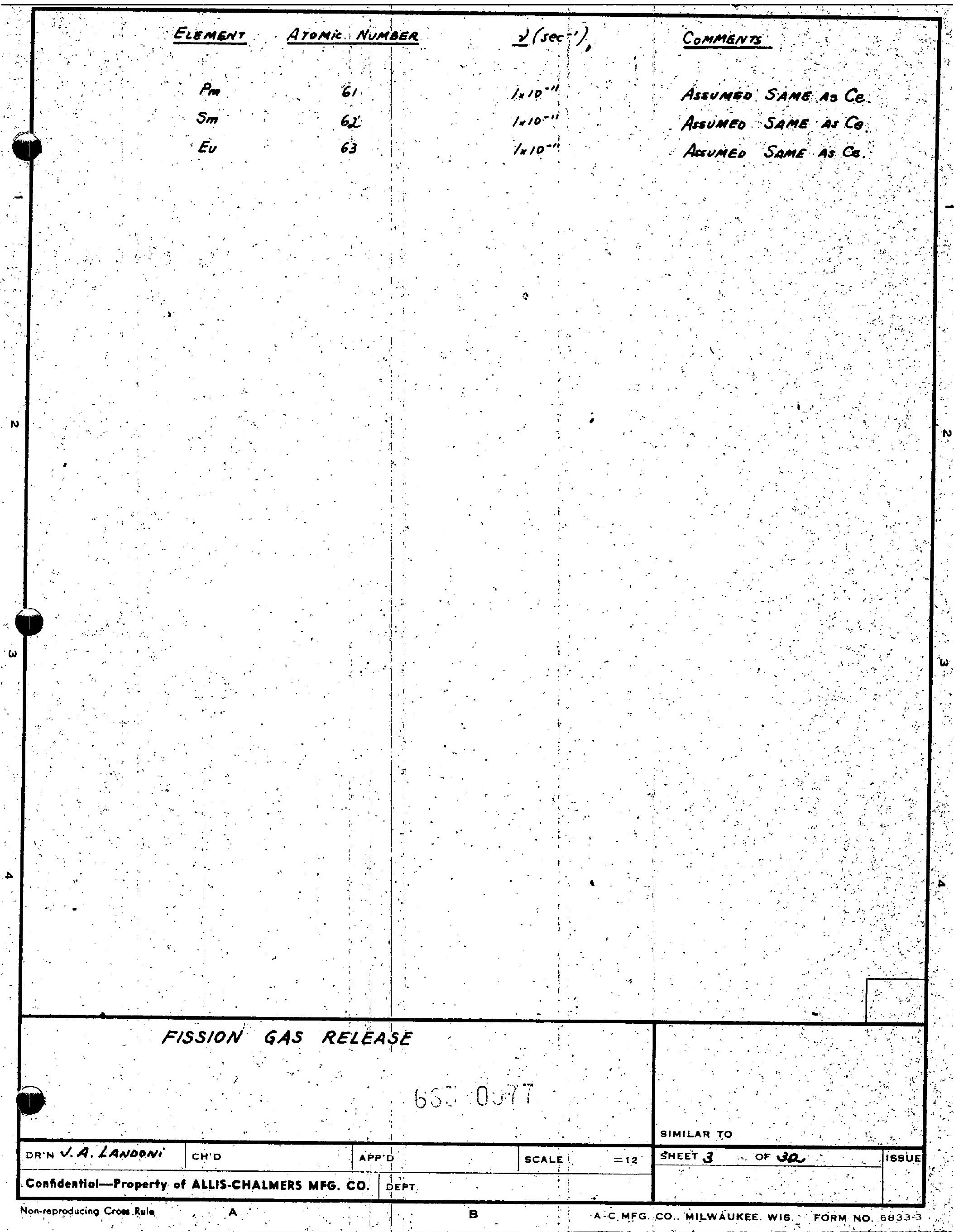




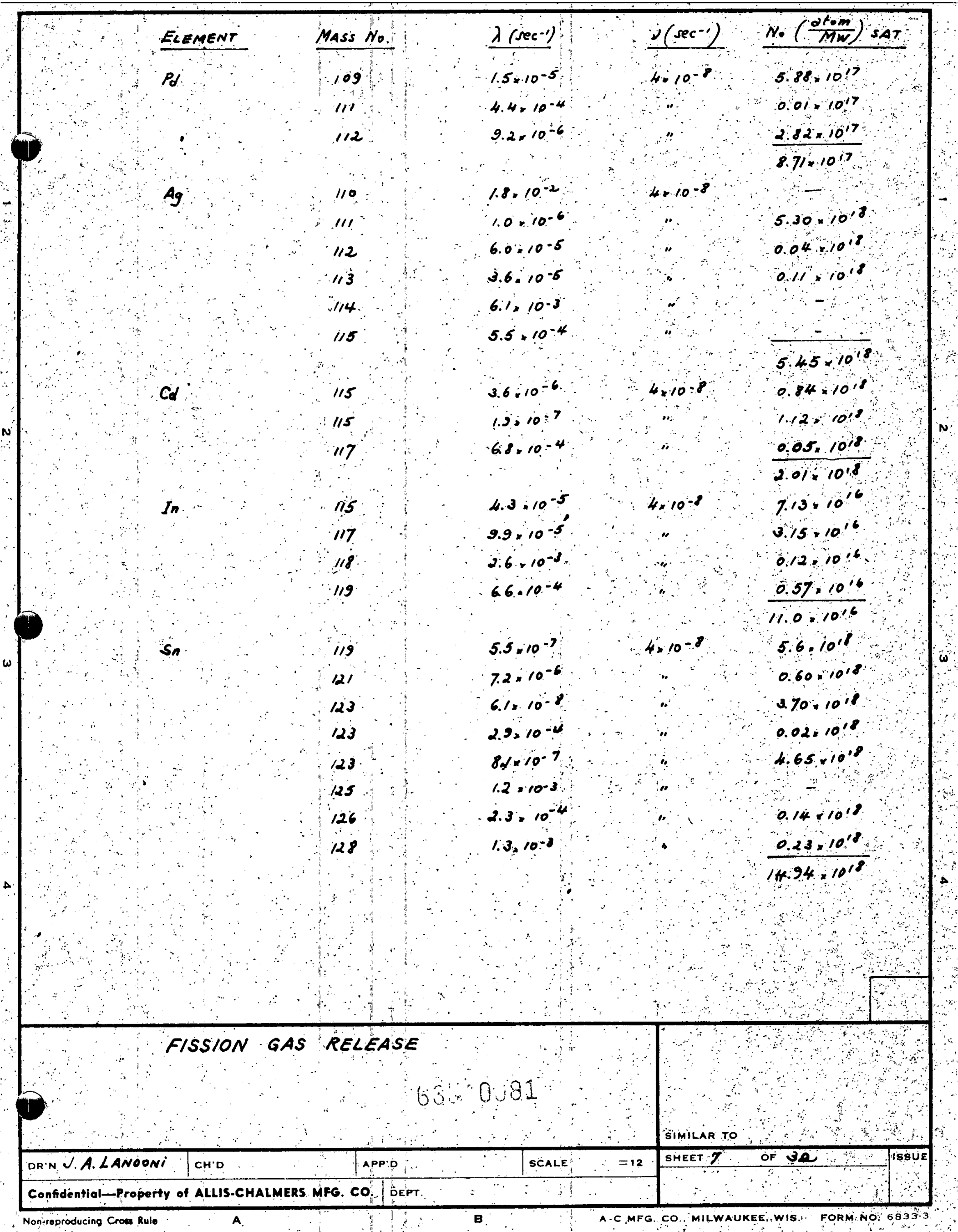




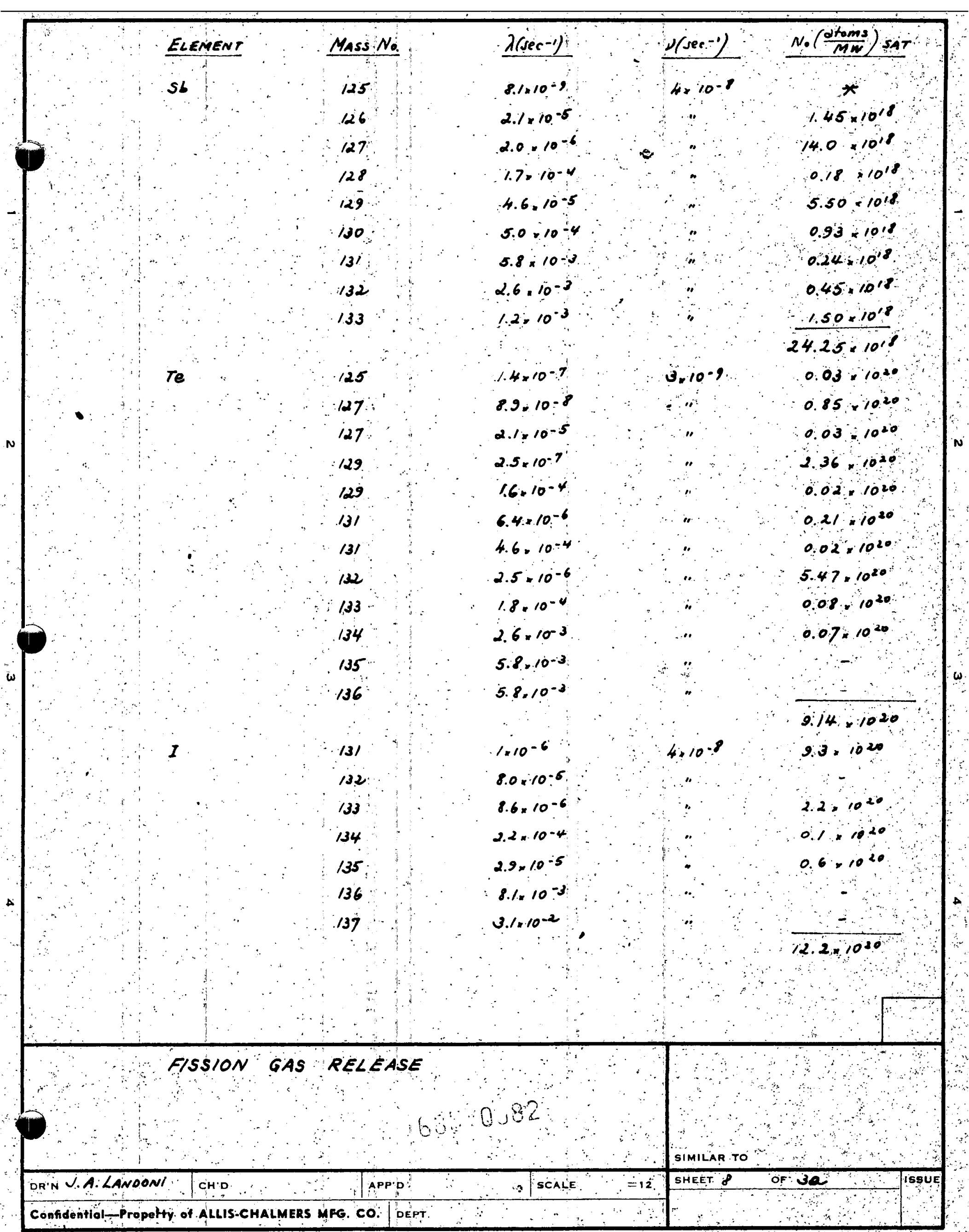




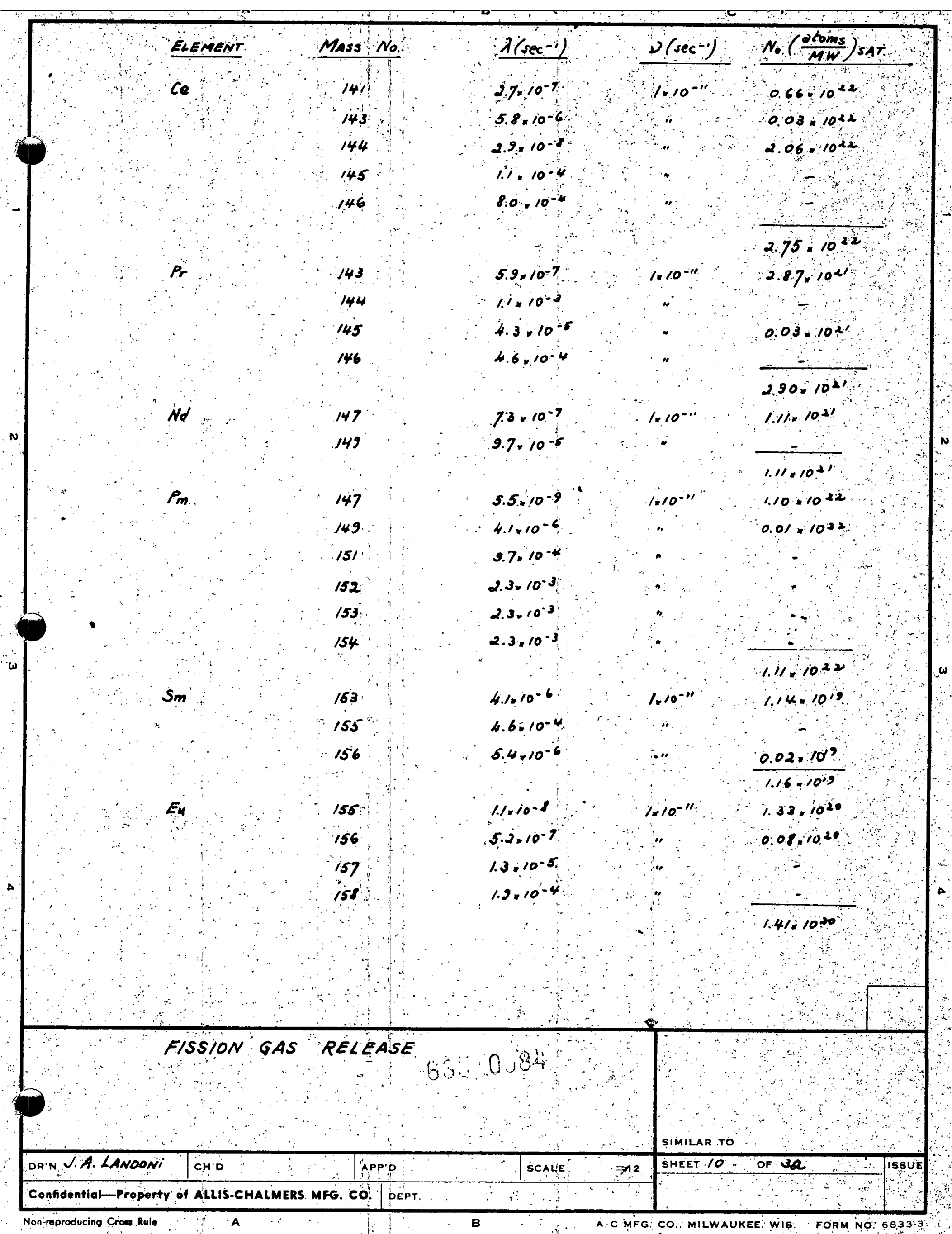


Se $=79$

$N_{0}=\frac{\left(3.7 \times 10^{-4}\right)\left(3.1 \times 10^{16}\right)\left(3.4 \times 10^{-13}\right)}{\left(1+10^{-7}+3.4+10^{-13}\right)} / \frac{1-\left(34 \times 10^{-13)\left(0.978 \times 10^{8}\right)}\right.}{\left(3.4 \times 10^{10}\right.}$

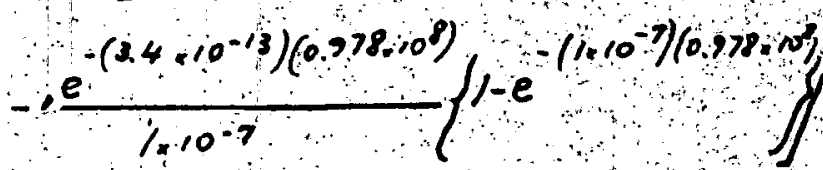

Ar-85

$N_{0}=3.42=10^{15}$

$N_{0}=181.1020$

a...

( $)(4.1 \times 107)(0.978109)$

Sr.90

$N_{0}=\frac{\left(3.1 \cdot 10^{-7}\right)\left(3.1 \times 10^{16}\right)\left(4.1 \times 10^{-7}\right)}{\left(1.10^{-7}+4.1 \times 10^{-7}\right)} / \frac{1-e}{4.1 \times 10^{-9}}$

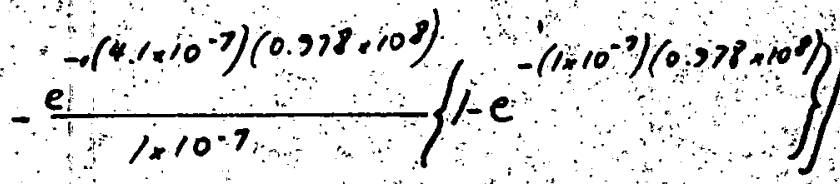

$N_{0}=1.88 \cdot 10^{.6}$

Ru-106

$N_{0}=\frac{(0.05)\left(3.1+10^{16}\right)\left(1.1 \times 10^{-9}\right)}{\left(7 \times 10^{\left.-10^{2}+1.1 \times 10^{-3}\right)}\right.}=\frac{1-e^{(1.1 \times 109)(0.87}}{\quad 11.10^{-9}}$

$-\frac{-(1.1 \times 10-9)(0.978 \times 108)}{7 \times 10^{-10}}\left\{1-e^{-\left(7 \times 10^{-10}\right)\left(0.978 \times 10^{8}\right)}\right\}$

$N_{0}=7: 48 \times 10^{2}$ 


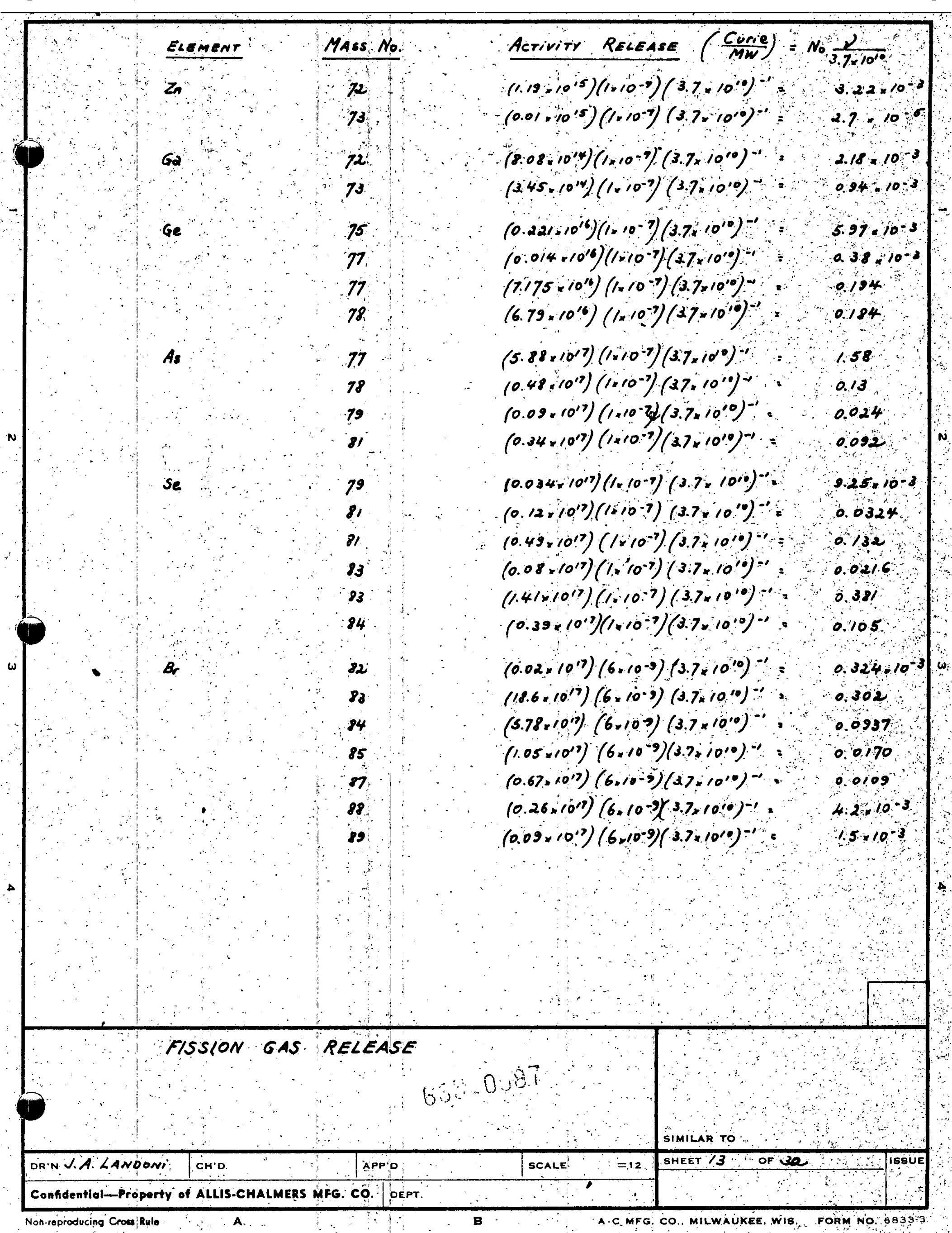




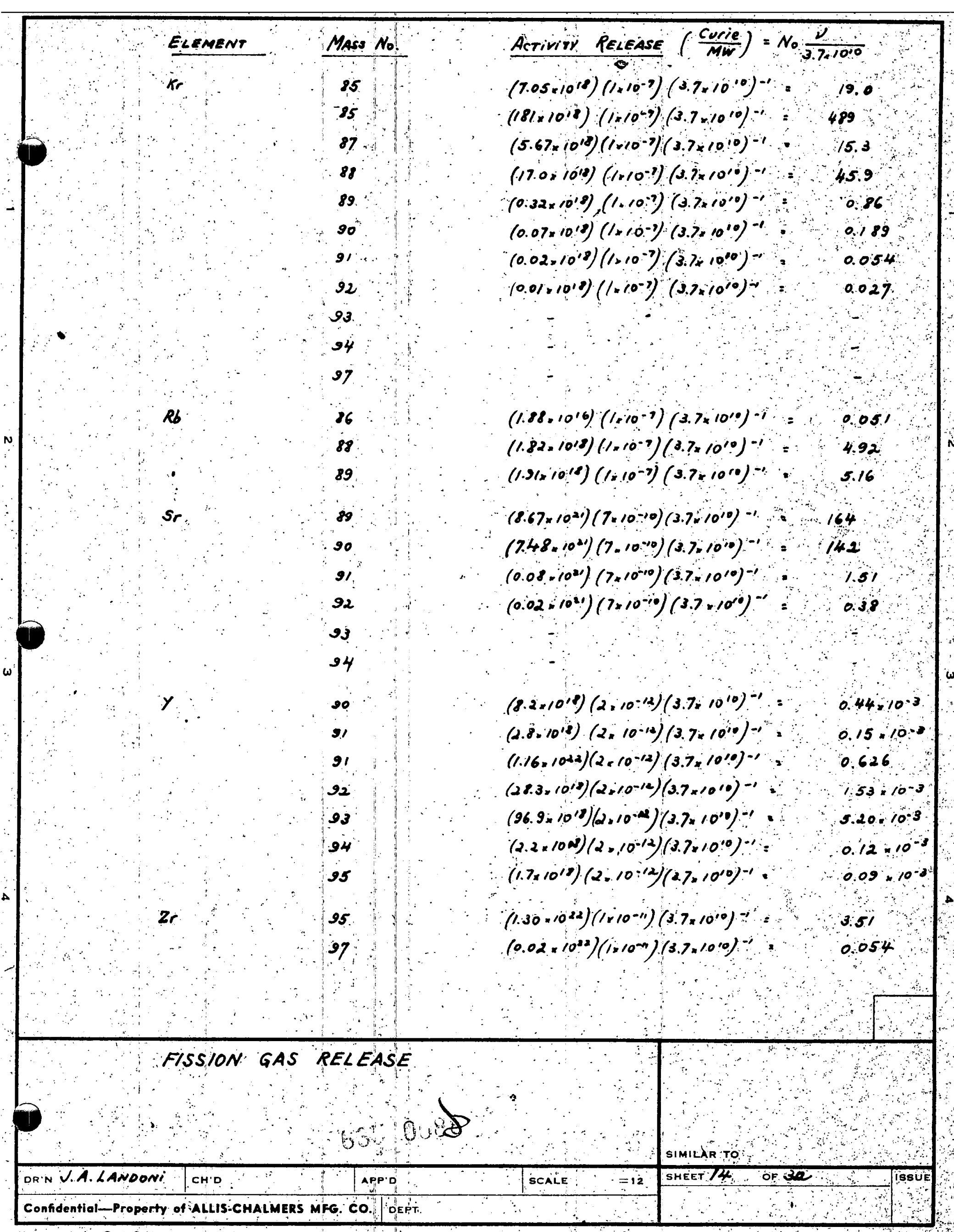




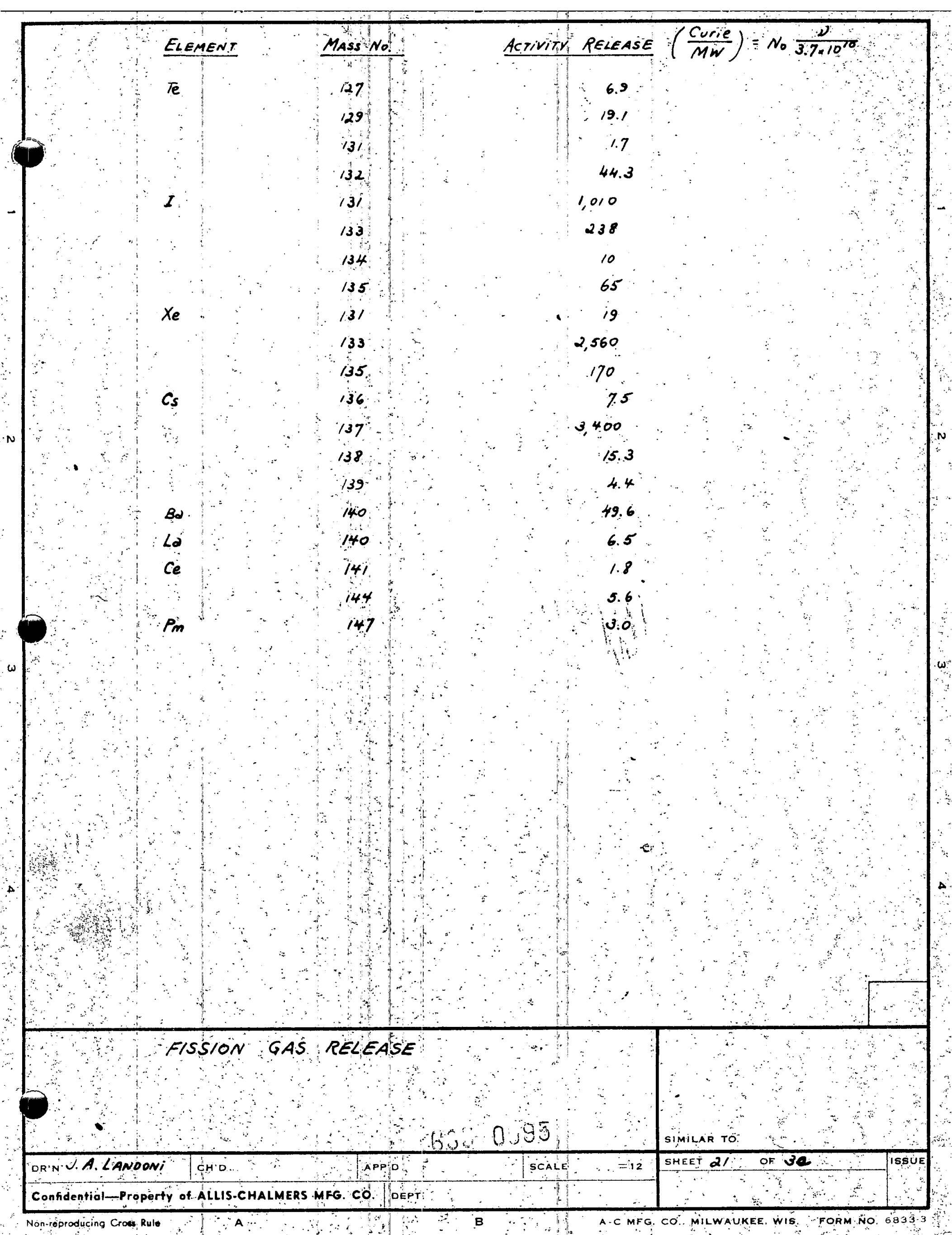




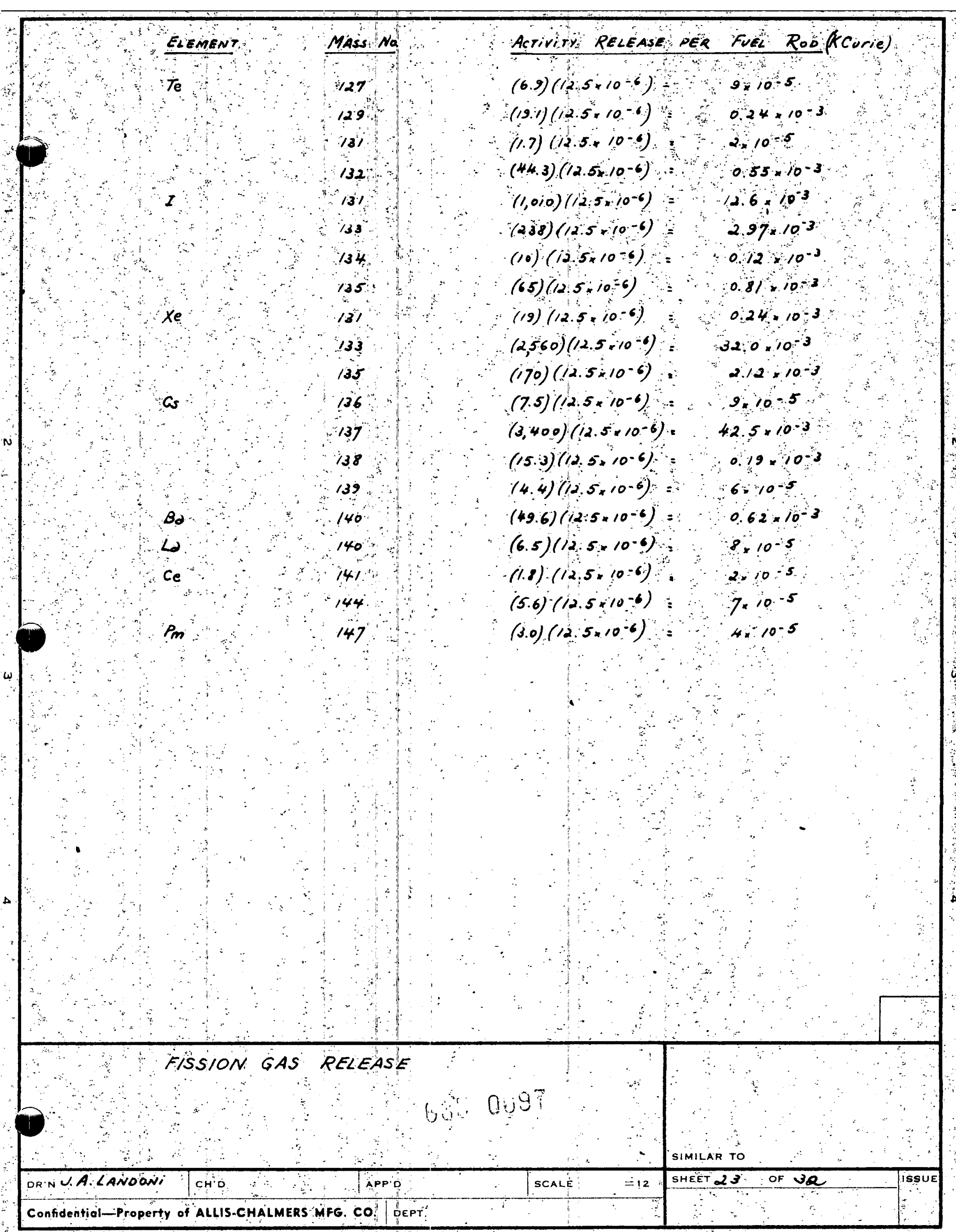




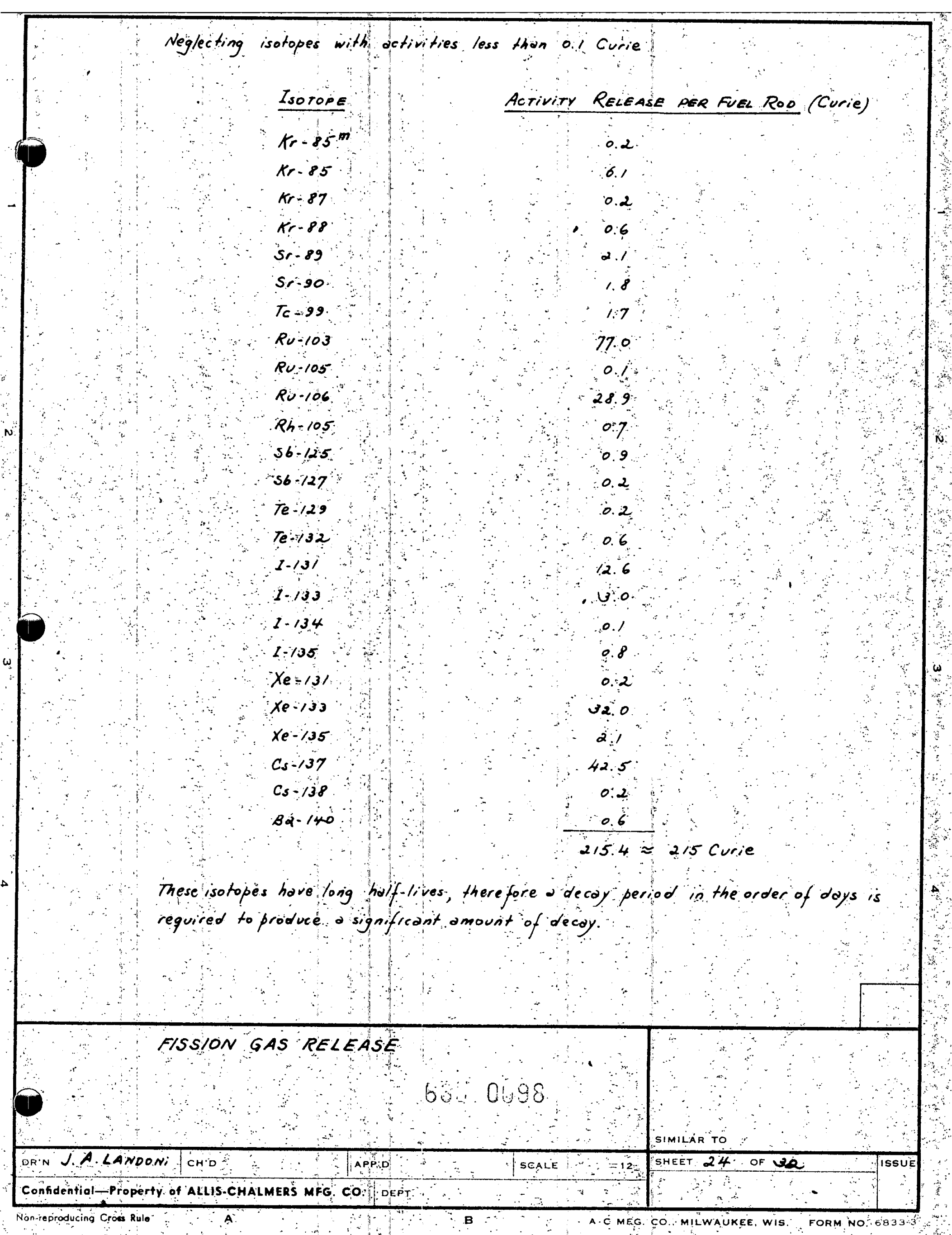




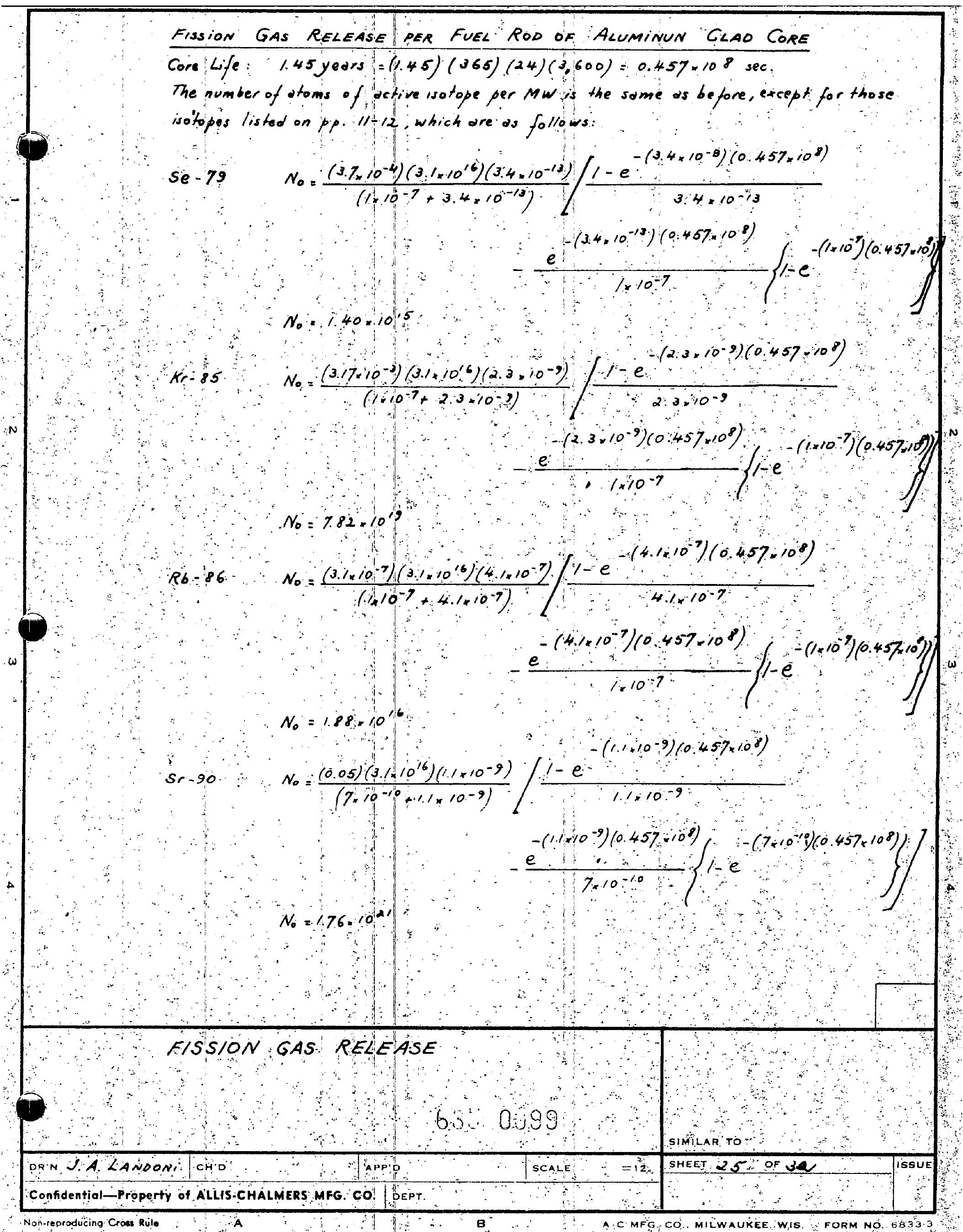




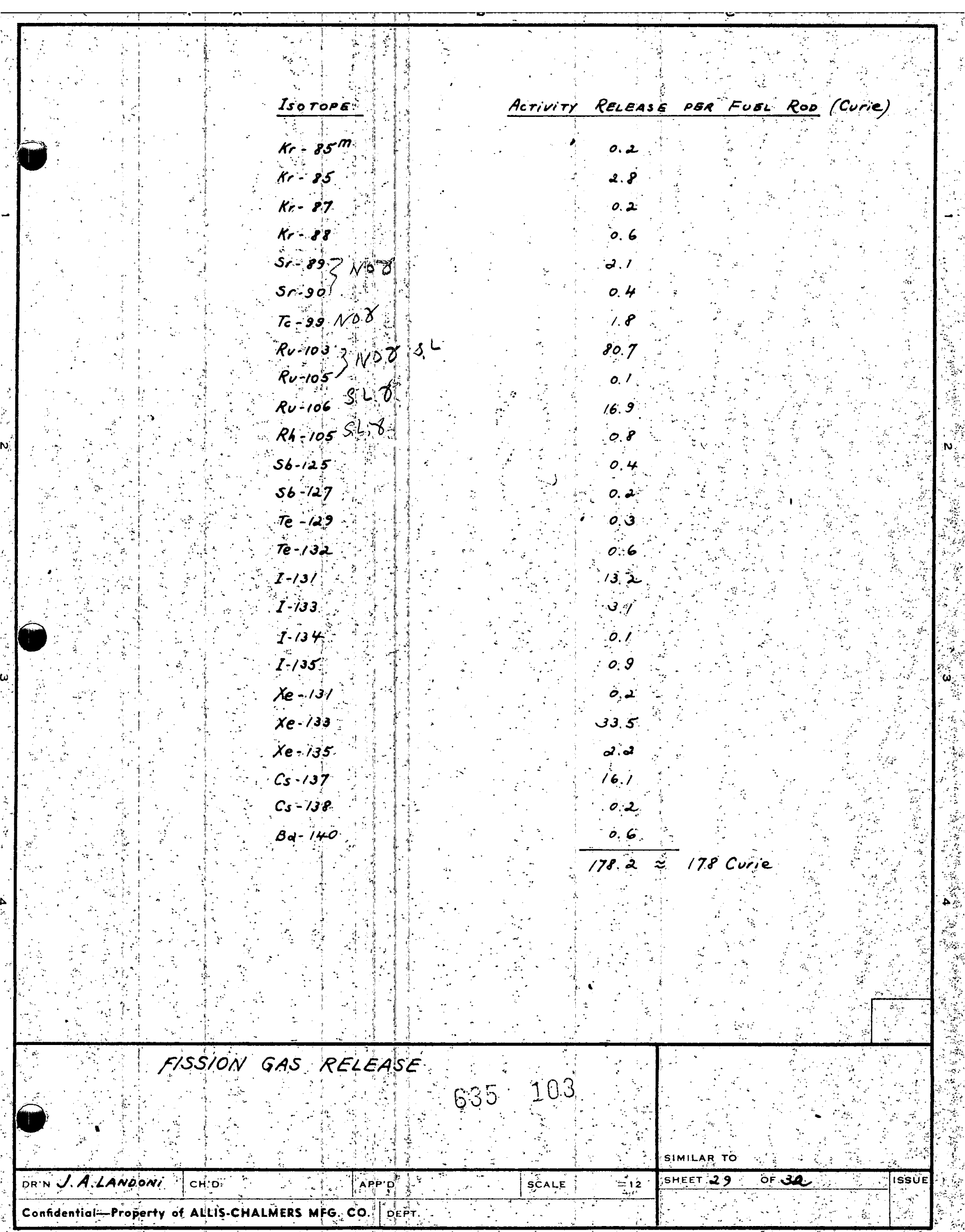




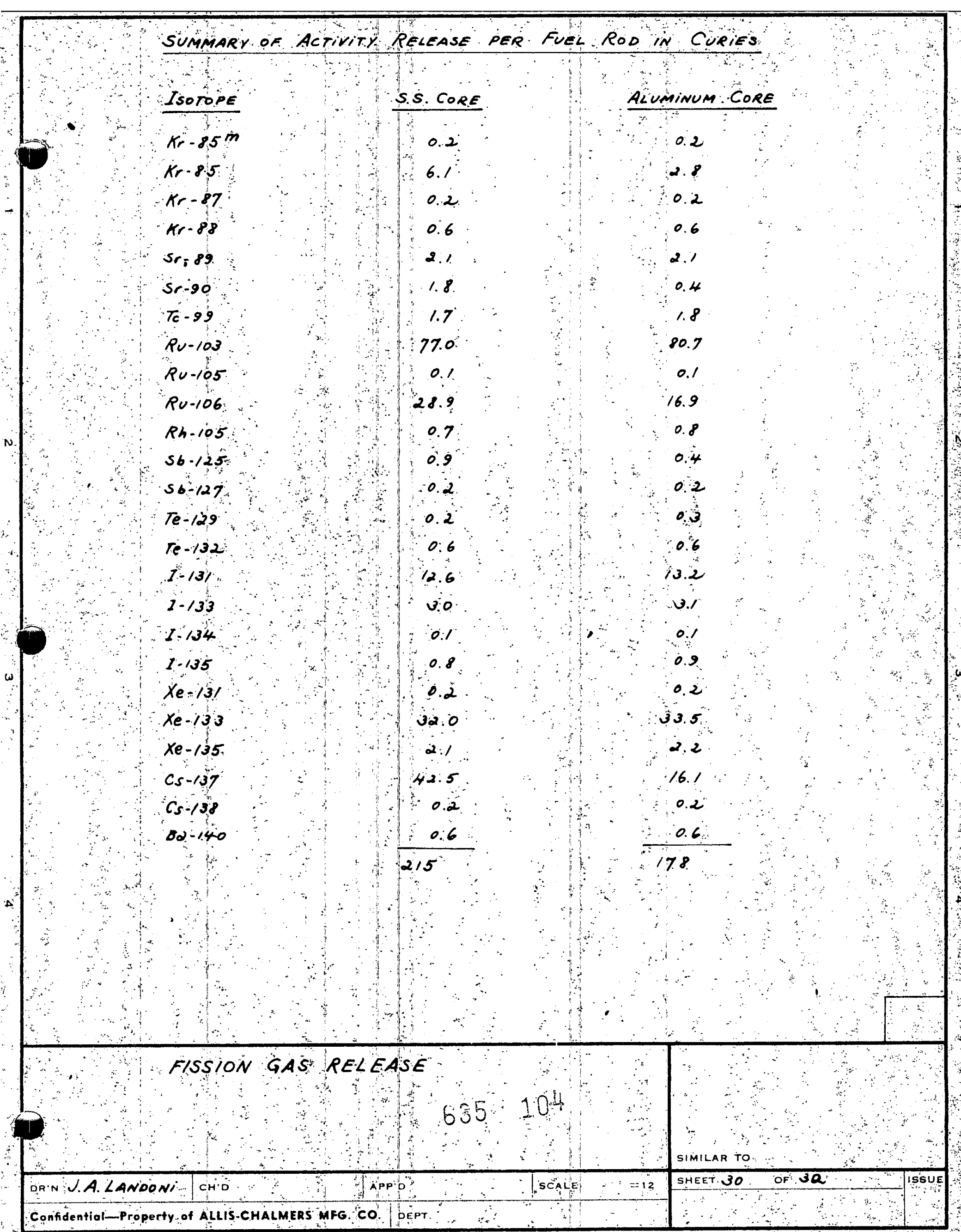


